\title{
QUANTUM MULTIPOLE RADIATION
}

\author{
ALEXANDER S. SHUMOVSKY \\ Physics Department, Bilkent University, Bilkent, Ankara, Turkey
}

There are some points which are as dark as ever. But we have so much that it will be our own fault if we cannot get the rest.

— Sir Arthur Conan Doyle, The Second Stain

\section{CONTENTS}

I. Introduction

II. Quantum Multipole Field
A. Classical Electromagnetic Field
B. Quantum Electromagnetic Field
C. Summary

III. Atom-Field Interaction
A. Multipole Jaynes-Cummings Model
B. The $S U(2)$ Atomic Phase States
C. The EPR Paradox and Entanglement
D. Summary

IV. Quantum Phase of Multipole Radiation
A. Conservation of Angular Momentum in the Process of Radiation
B. Dual Representation of Dipole Photons
C. Structure of Radiation Phase
D. Radiation Phase in Jaynes-Cummings Model
E. Radiation Phase and Pegg-Barnett Quantum Phase
F. Radiation Phase and Mandel's Operational Approach
G. Phase Properties of Radiation in Fabry-Pérot Resonator
H. Summary

V. Polarization Properties of Multipole Radiation
A. Polarization of Classical Field
B. Polarization of Quantum Radiation
C. Spatial Properties of Polarization
D. Operator Polarization Matrix in the Proper Frame
E. Summary 
VI. Measurement, Locality, and Causality
A. Measurement and Photon Localization
B. Causality in the Two-Atom Hertz Experiment
C. Polarization Measurements
D. Nondemolition Polarization Measurement
E. Summary

VII. Conclusion

Acknowledgments

References

\section{INTRODUCTION}

Before we start to investigate that, let us try to realize what we do know, so as to make the most of it, and to separate the essential from the accidental.

- Sir Arthur Conan Doyle, The Priory School

Since the pioneering paper by Dirac [1], the formalism of quantum electrodynamics (QED) has been based on the use of the photon creation and annihilation operators, forming a representation of the Weyl-Heisenberg algebra, and on the notion of the electromagnetic vacuum state [2-4]. As far as a denumerable set of Fock number states can be generated from the vacuum state by successive action of the creation operator, one can choose to interpret the electromagnetic vacuum as a "physical system" ready for support of any electromagnetic radiation.

It is not heretical to consider the electromagnetic vacuum as a "physical system." In fact, it manifests some physical properties and is responsible for a number of important effects. For example, the field amplitudes continue to oscillate in the vacuum state. These zero-point oscillations cause the spontaneous emission [1], the natural linebreadth [5], the Lamb shift [6], the Casimir force between conductors [7], and the quantum beats [8]. It is also possible to generate quantum states of electromagnetic field in which the amplitude fluctuations are reduced below the symmetric quantum limit of zero-point oscillations in one quadrature component [9].

In spite of the great success of QED, there still are a number of unclear principal problems [10-15]. Leaving aside the detailed discussion of foundations of QED, we shall concentrate here on the problems of localization of photons and quantum phase of electromagnetic radiation, which have attracted a great deal of interest.

The point is that the photon creation and annihilation operators are defined in QED as nonlocal objects. In other words, the photon number operator gives the total number of photons in the volume of quantization without specification of their spacetime location $[14,15]$. Moreover, it has been proved by Newton and Wigner [16] that no position operator can exist for the photon. There is a widespread belief that the maximum precise localization appears in the form of 
a wavefront [17]. At the same time, the specific falloff of the photon energy density and of the photodetection rate can be interpreted as the photon localization in space $[18,19]$.

Perhaps the most evident and bright example of photon localization is provided by the photodetection process, when a photon is transformed into electronic signal in the sensitive element of the detecting device [20]. Such a localization is usually described in the operational way (in terms of what can be measured by a macroscopic detector) through the use of the so-called configuration number operator, determining the number of photons in the cylindrical volume $\sigma c \Delta t$, where $\sigma$ denotes the area of the sensitive element, $c$ is the light velocity, and $\Delta t$ is the detector exposition time [14,20]. Other interesting examples are provided by the localization in photonic crystals [21] and by the emission and absorption of radiation by atoms and molecules [22].

We now stress that, in the usual treatment of photon localization, the radiation field is considered as though it consist of the plane waves of photons [14-20]. In reality, the radiation emitted by the atomic transitions corresponds to the multipole photons [23] represented by the quantized spherical waves [2].

Although the classical plane and spherical waves are equivalent in the sense that they both form complete orthogonal sets of solutions of the homogeneous Helmholtz wave equation [24,25], there is a strong qualitative difference between the two quantum representations. The plane waves of photons correspond to the running-wave solutions of the homogeneous Helmholtz wave equation in a large but finite cubic cavity with periodic boundary conditions $[1,2,14,15]$. This choice of the boundary conditions corresponds to the translational symmetry of solutions and leads to the states of photons with given linear momentum $[3,10,11]$. In turn, the solution of the homogeneous Helmholtz wave equation in terms of spherical waves assumes the existence of a singular point, corresponding to an atom (source or absorber of radiation) whose size is small with respect to the wavelength [24-26]. In this case, the boundary conditions correspond to the rotational symmetry and lead to the states of photons with given angular momentum $[2,4,27]$. Since the components of linear and angular momenta do not commute, the two representations of quantum electromagnetic field correspond to physical quantities that cannot be measured at once.

The simplest way to show the principal difference between the representations of plane and multipole photons is to compare the number of independent quantum operators (degrees of freedom), describing the monochromatic radiation field. In the case of plane waves of photons with given wavevector $\vec{k}$ (energy and linear momentum), there are only two independent creation or annihilation operators of photons with different polarization $[2,14,15]$. It is well known that QED (quantum electrodynamics) interprets the polarization as given spin state of photons [4]. The spin of photon is known to be 1 , so that there are three possible spin states. In the case of plane waves, projection of spin on the 
direction of $\vec{k}$ is forbidden because of the translational invariance, and hence only two transversal polarizations are allowed [4].

In turn, the monochromatic multipole photons are described by the scalar wavenumber $k$ (energy), parity (type of radiation either electric or magnetic), angular momentum $j=1,2, \ldots$, and projection $m=-j, \ldots, j[2,26,27]$. This means that even in the simplest case of monochromatic dipole $(j=1)$ photons of either type, there are three independent creation or annihilation operators labeled by the index $m=0, \pm 1$. Thus, the representation of multipole photons has much physical properties in comparison with the plane waves of photons. For example, the third spin state is allowed in this case and therefore the quantum multipole radiation is specified by three different polarizations, two transversal and one longitudinal (with respect to the radial direction from the source) $[27,28]$. In contrast to the plane waves of photons, the projection of spin is not a quantum number in the case of multipole photons. Therefore, the polarization is not a global characteristic of the multipole radiation but changes with distance from the source [22].

Another very important difference between the plane and multipole photons consists in the character of zero-point oscillations of the field strengths [29]. We shall show here that, unlike the former case with spatially homogeneous zeropoint oscillations, the multipole vacuum noise strongly depends on the distance from the singular point (atom). It is not an unexpected result. In fact, zero-point oscillations reflect the structure of the electromagnetic vacuum state, which, in turn, depends on the boundary conditions for the homogeneous Helmholtz wave equation [3]. Let us note in this connection that the possible influence of an atom on the electromagnetic vacuum state in the absence of radiation has been discussed in QED for a long time [30,31]. It should be stressed that the spatial inhomogeneity of the multipole vacuum noise can be very important for prognosis of experiments with trapped atoms [32] and single-atom laser [33], especially in the engineered entanglement in the atom-photon systems [32].

We now note that, since the 1990s entanglement has been recognized as one of the most fundamental features of quantum systems as well as an important tool of quantum communication and information processing [34]. One of the promising ways in the engineered entanglement is represented by the so-called two-photon polarization entanglement (see Sec. 12.14 in Ref. 14). In this case, the cascade decay of an atomic transition leads to the creation of two entangled photons with different polarizations and different directions of propagation. Therefore, an adequate estimation of the vacuum noise in atom-photon interactions seems to be of great importance.

While the simplified picture based on the model of plane waves of photons, neglecting the presence of sources and absorbers, is incapable of describing the photon localization, we show here that the use of the rich physical properties of multipole photons leads to an adequate description of localization in the atom- 
field interaction processes as well as in conventional photodetection. We note that the causal relation between the boundary conditions for the homogeneous Helmholtz wave equation and photon localization has been discussed since the late 1990s [19,22,29,35].

The representation of multipole photons is also useful in the investigation of the quantum phase problem [36]. In the pioneering paper [1] on the quantization of electromagnetic field, Dirac first postulated the existence of a Hermitian phase operator defined by the polar decomposition of the annihilation operator and conjugated to the photon number operator. Later it was realized that the Dirac's phase operator cannot be considered as a properly defined Hermitian operator, describing the quantum phase properties of electromagnetic radiation (for reviews, see Refs. 14 and 37-40). In particular, Susskind and Glogower [41] emphasized that the main difficulty in the correct definition of the phase operator arises because the spectrum of the number operator is bounded from below. An extension of the eigenvalue spectrum to negative values allows for the correct mathematical construction of the Hermitian phase operator [42,43], which leads to nonphysical states. An attempt to use the cosine and sine of the phase operators rather than the quantum phase operator has also been discussed [44].

A way to overcome the difficulties in the definition of the Hermitian phase operator has been proposed by Pegg and Barnett $[40,45]$. Their method is based on a contraction of the infinite-dimensional Hilbert-Fock space of photon states $\mathscr{H}$. Within this method, the quantum phase variable is determined first in a finite $s$-dimensional subspace of $\mathscr{H}$, where the polar decomposition is allowed. The formal limit $s \rightarrow \infty$ is taken only after the averages of the operators, describing the physical quantities, have been calculated. Let us stress that any restriction of dimension of the Hilbert-Fock space of photons is equivalent to an effective violation of the algebraic properties of the photon operators and therefore can lead to an inadequate picture of quantum fluctuations [46].

Perhaps, the most important result in the field of quantum phase problem was obtained by Mandel et al. [47] within the framework of the operational approach. According to their analysis, there is no unique quantum phase variable, describing universally the measured phase properties of light. This very strong statement has obtained a totally convincing confirmation in a number of experiments $[47,48]$. The results of the operational approach can be interpreted with the aid of the method based on the special quasiprobability distribution functions [49].

Generally speaking, the quantum phase variables can be divided into two classes. First, we have the pure operational phases that are completely determined by the scheme of measurement. This has no contradiction with the existence of an intrinsic quantum-dynamical variable responsible for the phase properties of light [50]. In addition, there might be some inherent quantum 
phases related to the quantum properties of photons. Since any photon can be specified by its energy, angular momentum, and/or linear momentum, the inherent phase should be determined by either the angular or linear momenta, as the energy is a scalar. The former is connected with the spin states and hence, with the polarization of radiation field. The latter can lead to some "geometric" phase, which, for example, can be measured as the phase difference between two plane waves emitted by one source in opposite directions.

It is well known that the angular momentum of a quantum mechanical system is specified by a representation of the $S U(2)$ algebra. If the corresponding enveloping algebra contains a uniquely defined scalar (the Casimir operator), the polar decomposition of the angular momentum can be obtained [51]. This polar decomposition determines a dual representation of the $S U(2)$ algebra expressed in terms of so-called phase states [51]. In particular, the Hermitian operator of the $S U(2)$ quantum phase can be constructed [51].

Although the angular momentum of quantum multipole radiation is well defined in terms of the multipole photon operators of creation and annihilation, the direct polar decomposition of the corresponding $S U(2)$ subalgebra in the Weyl-Heisenberg algebra is impossible. The point is that this $S U(2)$ subalgebra has no isotype representation [52]. This means that the Casimir operator (scalar) cannot be uniquely determined in the whole Hilbert-Fock space of photon states. Hence, the quantum phase of the angular momentum of multipole photons cannot be determined by a method proposed [51] as valid for the quantum mechanical systems.

An approach focused on overcoming this difficulty has been developed $[36,46,53,54]$. The main idea, which seems to be a very natural one, is to consider the radiation of a given quantum source (atom or molecule) rather than a source-free electromagnetic field represented by the plane waves. Even in the classical picture, the multipole radiation can be determined completely only if the source functions, describing a local source at the origin, are known [25]. Within the quantum picture where the atom-field interaction is described in terms of the perturbation theory [26], we can take into account the source dependence of radiation using the conservation laws. In particular, the conservation of angular momentum in the process of radiation [26] permits us first to define the $S U(2)$ quantum phase of the atomic transition, following the method by Vourdas [51], an then to construct an operator complement of the atomic cosine and sine operators with respect to the integrals of motion in the whole atom-field system [36].

Many attempts have been made to define the quantum phase of light via the angular momentum (e.g., see Ref. 55 and references cited therein). The new element of our approach $[36,46,53,54]$ is that we determine the quantum phase of radiation via the quantum phase of the angular momentum of its source. 
Let us stress that the electromagnetic vacuum state has no phase at all. This is the same as saying that the vacuum state is degenerated with respect to the phase or that the phase is distributed uniformly over the vacuum [14,15]. The degeneration is taken off in the process of creation of the photon by atomic transition. Thus, it seems quite logic to assume that the inherent quantum phase of photons is generated by the source [36,46]. Definitely, this is not an unusual assumption. Actually, the classical amplitudes of the multipole field are completely determined by the source functions, describing the charge density, current density, and magnetization [25]. Hence, the multipole photon operators, which are obtained by the quantization of classical amplitudes $[1,2]$, are also specified by the source [56].

We also note that, in contrast to the Pegg-Barnett formalism [45], we consider an extended space of states, including the Hilbert-Fock state of photons as well as the space of atomic states [36,46,53,54]. The quantum phase of radiation is defined, in this case, by mapping of corresponding operators from the atomic space of states to the whole Hilbert-Fock space of photons. This procedure does not lead to any violation of the algebraic properties of multipole photons and therefore gives an adequate picture of quantum phase fluctuations [46].

We provide here a review of investigations of the photon localization and quantum phase problems based on the use of the representation of multipole photons. Section II presents a general consideration of the field quantization. In particular, we compare the zero-point oscillations of the plane and multipole waves of photons and show that the vacuum noise is concentrated in some vicinity of atoms. In Section III we discuss the atom-field interaction leading to the multipole radiation and consider the $S U(2)$ quantum phase representation of atomic variables. Here we also discuss a connection between the $S U(2)$ quantum phase states and entanglement phenomenon. In Section IV we describe the quantum phase of multipole radiation caused by the angular momentum conservation in the process of radiation. We compare this approach with the Pegg-Barnett formalism and with Mandel's operational approach. In Section V we consider the quantum polarization properties of multipole radiation. Then, in Section VI, we discuss the photon localization, quantum measurements, and causality. To simplify the reading, we supplement each section by a brief summary. A general conclusion and the implications of this work are presented in Section VII.

\section{QUANTUM MULTIPOLE FIELD}

We must not think of the things that we could do with, but only of the things we can't do without. 


\section{A. Classical Electromagnetic Field}

An arbitrary free classical electromagnetic field is described by the vector potential $\overrightarrow{\mathscr{A}}(\vec{r})$, which obeys the wave equation [14,24,25]

$$
\nabla^{2} \overrightarrow{\mathscr{A}}-\frac{1}{c^{2}} \frac{\partial^{2} \overrightarrow{\mathscr{A}}}{\partial t^{2}}=0
$$

and Coulomb gauge condition

$$
\vec{\nabla} \cdot \overrightarrow{\mathscr{A}}=0
$$

The field strengths are then defined as follows:

$$
\overrightarrow{\mathscr{E}}=-\frac{1}{c} \frac{\partial \overrightarrow{\mathscr{A}}}{\partial t}, \quad \overrightarrow{\mathscr{B}}=\vec{\nabla} \times \overrightarrow{\mathscr{A}}
$$

Equation (1) can be solved by separation of variables [24]:

$$
\overrightarrow{\mathscr{A}}(\vec{r}, t)=\sum_{\ell} q_{\ell}(t) \vec{u}_{\ell}(\vec{r})
$$

Employing (1) then gives the homogeneous Helmholtz wave equations of the form

$$
\begin{aligned}
\frac{d^{2} q_{\ell}}{d t^{2}}+\omega_{\ell}^{2} q_{\ell} & =0 \\
\nabla^{2} \vec{u}_{\ell}+\frac{\omega_{\ell}^{2}}{c^{2}} \vec{u}_{\ell} & =0
\end{aligned}
$$

where $\omega_{\ell}$ are some constants, arising from the separation of variables [24]. Solution of the first equation in (5) gives the harmonic time dependence $q_{\ell}=\exp \left( \pm i \omega_{\ell} t\right)$. Because of the harmonic time dependence in (4), it is customary to represent the vector potential in terms of the positive and negative frequency parts:

$$
\vec{A}(\vec{r})=\vec{A}(\vec{r})+\vec{A}^{*}(\vec{r})
$$

where $\vec{A} \sim \exp (-i \omega t)$.

The energy density of the field is

$$
W(\vec{r})=\frac{1}{16 \pi}\left[\overrightarrow{\mathscr{E}}^{*}(\vec{r}) \cdot \overrightarrow{\mathscr{E}}(\vec{r})+\overrightarrow{\mathscr{B}}^{*}(\vec{r}) \cdot \overrightarrow{\mathscr{B}}(\vec{r})\right]
$$


In turn, the flux of energy is given by the real part of the complex Poynting vector

$$
\vec{S}(\vec{r})=\frac{1}{8 \pi} \vec{E}(\vec{r}) \times \vec{B}^{*}(\vec{r})
$$

where, according to (3), we obtain

$$
\vec{E}(\vec{r})=-i k \vec{A}(\vec{r}), \quad \vec{B}(\vec{r})=\vec{\nabla} \times \vec{A}(\vec{r})
$$

The angular momentum density of the field has the form [25]

$$
\vec{M}(\vec{r})=\frac{1}{4 \pi c} \vec{r} \times[\overrightarrow{\mathscr{E}}(\vec{r}) \times \overrightarrow{\mathscr{B}}(\vec{r})]
$$

One possible solution of the first equation in (5), corresponding to the plane waves, traveling along the $z$ axis and having the same amplitude and phase everywhere [24], has the form [14,24,25]

$$
\vec{u}_{\ell}(\vec{r})=\sum_{\ell} \sum_{\sigma=x, y} \vec{e}_{\ell \sigma} e^{i \vec{k}_{\ell} \cdot \vec{r}} a_{\ell \sigma}+\text { c.c }
$$

(where c.c. denotes complex conjugates). Here $a_{\ell \sigma}$ are the complex field amplitudes, $\vec{e}_{x, y}$ are the unit vectors of polarization which, due to the Coulomb (guage) condition (2), obey the relation

$$
\forall \ell \quad \vec{e}_{x, y} \cdot \vec{k}_{\ell}=0
$$

and $k_{\ell}^{2}=\omega_{\ell}^{2} / c^{2}$. Employing (3), (6), and (10) then gives

$$
\begin{aligned}
& E_{x}(\vec{r})=i \sum_{k} k A_{k x}(\vec{r})=B_{y}(\vec{r}) \\
& E_{y}(\vec{r})=i \sum_{k} k A_{k y}(\vec{r})=-B_{x}(\vec{r})
\end{aligned}
$$

To simplify the notations, we omit the index $\ell$ here. According to (10), we have

$$
A(\vec{r})=\sum_{k} \gamma_{k} \sum_{\sigma=x, y} \vec{e}_{k \sigma} e^{i \vec{k} \cdot \vec{r}} a_{k \sigma} e^{-i \omega t}
$$

where $\gamma_{k}$ is the normalization factor. Another possible solution of the homogeneous Helmholtz wave equation (5) convenient for electromagnetic boundary-value problems possessing spherical symmetry properties is provided 
by the spherical waves [24,25]. In this case, it is supposed that there is a singular point at the origin, corresponding to a localized source distribution or to an absorber (in the case of incoming spherical waves).

In the spherical coordinates $x=r \sin \theta \cos \phi, y=r \sin \theta \sin \phi$, and $z=r \cos \theta$, the second equation in (5) takes the form [24]

$$
\frac{\partial^{2} u_{\ell}}{\partial r^{2}}+\frac{2}{r} \frac{\partial u_{\ell}}{\partial r}+\frac{1}{r^{2} \sin \theta} \frac{\partial}{\partial \theta}\left(\sin \theta \frac{\partial u_{\ell}}{\partial \theta}\right)+\frac{1}{r^{2} \sin ^{2} \theta} \frac{\partial^{2} u_{\ell}}{\partial \phi^{2}}+\frac{\omega_{\ell}^{2}}{c^{2}} u_{\ell}=0
$$

A corresponding solution can be found by the separation of variables $u=R(r) \Theta(\theta) \Phi(\phi)$ in the mode function in (5), which yields the following set of ordinary differential equations [24]:

$$
\begin{aligned}
\frac{d^{2} R}{d r^{2}}+\frac{2}{r} \frac{d R}{d r}+\left[\frac{\omega^{2}}{c^{2} r^{2}}-j(j+1)\right] R & =0 \\
\frac{1}{\sin \theta} \frac{d}{d \theta}\left(\sin \theta \frac{d \Theta}{d \theta}\right)+\left[j(j+1)-\frac{m^{2}}{\sin ^{2} \theta}\right] \Theta & =0 \\
\frac{d^{2} \Phi}{d \phi^{2}}+m^{2} \Phi & =0
\end{aligned}
$$

The solution of these equations is represented by certain combinations of spherical Bessel or Hankel functions and spherical harmonics [24-26].

To establish contact with the quantum picture, consider the so-called helicity basis [27]

$$
\vec{\chi}_{ \pm}=\mp \frac{\vec{e}_{x} \pm i \vec{e}_{y}}{\sqrt{2}}, \quad \vec{\chi}_{0}=\vec{e}_{z}
$$

It is clear that $\left\{\vec{\chi}_{\mu}\right\}$ formally coincide with the three states of spin 1 of a photon. Therefore, one can choose to interpret $\vec{\chi}_{ \pm}$as the unit vectors of circular polarization with either positive or negative helicity, while $\vec{\chi}_{0}$ gives the linear polarization in the $z$ direction [27]. We note here that to within the sign at $\vec{\chi}_{ \pm}$the helicity basis (16) coincides with the so-called polarization basis frequently used in optics [57].

In the basis (16), any vector $\overrightarrow{\mathscr{A}}$ can be expanded as follows:

$$
\overrightarrow{\mathscr{A}}=\sum_{\mu=-1}^{1}(-1)^{\mu} \vec{\chi}_{-\mu} \mathscr{A}_{\mu}
$$


In this basis, for the positive-frequency part of the vector potential in (6) we get [2,24-27]

$$
\vec{A}_{\lambda}(\vec{r})=\sum_{k} \sum_{\mu} \sum_{j} \sum_{m=-j}^{j}(-1)^{\mu} \vec{\chi}_{-\mu} V_{\lambda k j m \mu}(\vec{r}) a_{\lambda k j m} e^{-i \omega t}
$$

Here $\lambda=E, M$ denotes the type of radiation, either electric or magnetic, index $j$ takes the values $1,2, \ldots$, and index $m=-j, \ldots, j$. The complex field amplitudes are defined in terms of the source functions, describing the local distribution of current and intrinsic magnetization [25]. The mode functions in (17) can be represented in the following form [2,26,27]:

$$
\begin{aligned}
V_{E k j m \mu}= & \gamma_{E k j}\left[\sqrt{j} f_{j+1}(k r)\langle 1, j+1, \mu, m-\mu \mid j m\rangle Y_{j+1, m-\mu}(\theta \phi)\right. \\
& \left.-\sqrt{j+1} f_{j-1}(k r)\langle 1, j-1, \mu, m-\mu \mid j m\rangle Y_{j-1, m-\mu}(\theta, \phi)\right] \\
V_{M k j m \mu}= & \gamma_{M k j} f_{j}(k r)\langle 1, j, \mu, m-\mu \mid j m\rangle Y_{j m}(\theta, \phi)
\end{aligned}
$$

Here $\gamma_{\lambda k j}$ is the normalization constant, $\langle\cdots \mid j m\rangle$ denotes the Clebsch-Gordon coefficient, and $Y_{\ell m}$ is the spherical harmonics. The radial contribution into the mode functions (18) depends on the boundary conditions as follows [24]

$$
f_{\ell}(k r)= \begin{cases}h_{\ell}^{(1)}(k r), & \text { outgoing spherical wave } \\ h_{\ell}^{(2)}(k r), & \text { incoming spherical wave } \\ j_{\ell}(k r), & \text { standing spherical wave }\end{cases}
$$

where $h_{\ell}^{(1,2)}$ denotes the spherical Hankel function of the first and second kinds, respectively, and $j_{\ell}$ is the spherical Bessel function [24,25].

Unlike the case of plane waves of photons, the multipole field (18) propagates as a uniformly expanding spherical shell rather than propagates along a given direction of $\vec{k}$. Instead of the symmetry relations (12), for the spherical waves of photons we get the following reciprocity relations $[2,27]$ :

$$
\begin{aligned}
\vec{E}_{E k j m} & =\vec{B}_{M k j m}=i k \vec{A}_{E k j m} \\
\vec{E}_{M k j m} & =-\vec{B}_{E k j m}=i k \vec{A}_{M k j m}
\end{aligned}
$$

\section{B. Quantum Electromagnetic Field}

The canonical quantization of the field has introduced by Dirac [1] (see also Refs. $2-4,10,11,14,15,26,27)$ is provided by the substitution of the photon operators, forming a representation of the Weyl-Heisenberg algebra, into the 
expression for the vector potential instead of the complex field amplitudes. For example, in the case of plane waves, described by the positive-frequency part of the vector potential (13), we get the following operator construction

$$
A(\vec{r})=\sum_{k} \sum_{\sigma=x, y} \sqrt{\frac{2 \pi \hbar c}{k V}} \vec{e}_{k \sigma} e^{i \vec{k} \cdot \vec{r}} a_{k \sigma}
$$

where $V$ is the volume of quantization, which is supposed to be a large cubic box with periodical boundary conditions. Here the harmonic time dependence is included into the photon operators that obey the commutation relations

$$
\left[a_{k \sigma}, a_{k^{\prime} \sigma^{\prime}}^{+}\right]=\delta_{k k^{\prime}} \delta_{\sigma \sigma^{\prime}}
$$

As a result of the translational symmetry along the $z$ direction, the plane waves of photons, described by (21) and (22), correspond to the states of the radiation field with given linear momentum

$$
\vec{P}=\sum_{k \sigma} \hbar \vec{k} a_{k \sigma}^{+} a_{k \sigma}
$$

where $\vec{k}=k \vec{e}_{z}$.

The multipole electromagnetic field "can be quantized in much the same way as plane waves" [2]. We have to subject the complex field amplitudes in the expansion (17) to the Weyl-Heisenberg commutation relations of the form

$$
\left[a_{\lambda k j m}, a_{\lambda^{\prime} k^{\prime} j^{\prime} m^{\prime}}^{+}\right]=\delta_{\lambda \lambda^{\prime}} \delta_{k k^{\prime}} \delta_{j j^{\prime}} \delta_{m m^{\prime}}
$$

Then, the positive-frequency part of the operator vector potential of the multipole radiation of a given type $\lambda$ takes the form $[2,27]$

$$
\vec{A}_{\lambda}(\vec{r})=\sum_{k} \sum_{\mu} \sum_{j} \sum_{m=-j}^{j}(-1)^{\mu} \vec{\chi}_{-\mu} V_{\lambda k j m \mu}(\vec{r}) a_{\lambda k j m}
$$

where the harmonic time dependence is again included into the definition of the photon operators of creation and annihilation. In the case of standing waves of photons in an ideal spherical cavity of volume $V$, the normalization factors in (18) take the form [2,27]

$$
\begin{aligned}
\gamma_{E k j} & =\sqrt{\frac{2 \pi \hbar c}{k V(2 j+1)}} \\
\gamma_{M k j} & =\sqrt{\frac{2 \pi \hbar c}{k V}}
\end{aligned}
$$


Within the quantum picture, the Clebsch-Gordon coefficients in (18) represent the vector addition of the spin and orbital parts of the total angular momentum of the field [2]. The indices $j$ and $m$ in (23) and (24) correspond to the angular momentum and projection of angular momentum on the quantization axis. The electric-type multipole radiation is interpreted as having the parity of state $(-1)^{j+1}$, while the magnetic-type multipole radiation is specified by the states with parity $(-1)^{j}$. Because of the spherical symmetry of solutions (17)-(19), the representation of spherical waves of photons (23)-(24) corresponds to the states of quantum multipole field with given angular momentum. Since the components of the linear and angular momenta do not commute with each other, the two representations (21)-(22) and (23)-(24) are different in principle. They correspond to the physical observables that cannot be measured simultaneously.

For both the plane and multipole waves of photons, the vacuum state can be defined by the stability condition in the same way $[3,4]$ :

$$
\begin{aligned}
& \forall k, \sigma \quad a_{k \sigma}|0\rangle=0 \\
& \forall \lambda, k, j, m \quad a_{\lambda k j m}|0\rangle=0
\end{aligned}
$$

Then, the corresponding Fock number states are defined as follows

$$
\begin{aligned}
\left|n_{k \sigma}\right\rangle & =\frac{\left(a_{k \sigma}^{+}\right)_{k \sigma}^{n}}{\sqrt{n_{k \sigma} !}}|0\rangle \\
\left|n_{\lambda k j m}\right\rangle & =\frac{\left(a_{\lambda k j m}^{+}\right)_{\lambda k j m}^{n}}{\sqrt{n_{\lambda k j m}}}|0\rangle
\end{aligned}
$$

where $n \geq 0$ is an integer.

As can be seen from the equations (21)-(22) and (23)-(24), there is an essential difference between the representations of plane and multipole waves of photons. In particular, a monochromatic plane wave of photons is specified by only two different quantum numbers $\sigma=x, y$, describing the linear polarization in Cartesian coordinates. In turn, the monochromatic multipole photons are described by much more quantum numbers. Even in the simplest case of the electric dipole radiation when $\lambda=E$ and $j=1$, we have three different states of multipole photons in (23) with $m=0, \pm 1$. Besides that, the plane waves of photons have the same polarization $\sigma$ everywhere, while the states of multipole photons have given $m$. It is seen from (24) that, in this case, the polarization described by the spin index $\mu$ can have different values at different distances from the singular point. In Section V we discuss the polarization properties of the multipole radiation in greater detail.

A more profound difference between the two representations can be traced in the properties of the zero-point oscillations. In fact, the energy operators 
obtained by quantization of (7) in the plane and spherical wave representations have the form [26-59]

$$
\begin{aligned}
& H_{\text {(plane) }}=\sum_{k, \sigma} \hbar \omega_{k}\left(a_{k \sigma}^{+} a_{k \sigma}+\frac{1}{2}\right) \\
& H_{\text {(multi) }}=\sum_{k} \hbar \omega_{k} \sum_{\lambda, j, m}\left(a_{\lambda j m}^{+} a_{\lambda j m}+\frac{1}{2}\right)
\end{aligned}
$$

Thus, the energies of the vacuum state are

$$
\begin{aligned}
& H_{\text {(plane) }}^{(\mathrm{vac})}=\sum_{k, \sigma} \frac{\hbar \omega_{k}}{2}=\sum_{k} \hbar \omega_{k} \\
& H_{(\mathrm{multi})}^{(\mathrm{vac})}=\sum_{k} \hbar \omega_{k} \sum_{\lambda, j, m} \frac{1}{2}=\sum_{k} \hbar \omega_{k}\left(\sum_{j}(2 j+1)\right)
\end{aligned}
$$

According to the definition of $k$, both expressions give an infinite energy and, at first sight, cannot be compared with each other. In fact, this infinity is inessential because of the following reason. The contribution of zero-point oscillations can be observed only via measurement which implies an averaging of physical quantities over a finite "volume of detection" and exposition time of detector. Such an averaging plays a part of filtration leading to a selection of a certain finite transmission frequency band [58]. It is then seen that, even if the filtration process leads to separation of the dipole photons only, the second term in (27) exceeds the first one 3 times. From the physical point of view, this result is caused by the more number of quantum degrees of freedom in the case of multipole photons.

Much more interesting and important result can be obtained from the consideration of the spatial properties of the vacuum fluctuations. The simplest example is provided by the calculation of vacuum average of the squared electric field strength $[58,59]$

$$
W(\vec{r})=\langle 0|\overrightarrow{\mathscr{E}} \cdot \overrightarrow{\mathscr{E}}| 0\rangle=k^{2}\langle 0|\overrightarrow{\mathscr{A}} \cdot \overrightarrow{\mathscr{A}}| 0\rangle
$$

obtained from (6) by the canonical quantization of the field. It follows from the definition of the vacuum state (25) that this expression can be put into the form

$$
W(\vec{r})=k^{2}\left\langle 0\left|\left[\vec{A}, \vec{A}^{+}\right]\right| 0\right\rangle=k^{2}\left[\vec{A}, \vec{A}^{+}\right]
$$

independent of the type of representation. Consider first the monochromatic plane waves of photons. Using (21) together with the commutation relations 
(22), we get

$$
W^{(\text {plane })}(\vec{r})=W^{(\text {plane })}=\sum_{k, \sigma} \frac{\hbar \omega_{k}}{2 V}, \quad \omega_{k}=k c
$$

Here $V$ has the same meaning as in (21). Thus, the zero-point fluctuations of the electric field strength of plane waves have the same magnitude at any space point. By construction, (28) describes the zero-point fluctuations in empty space.

In turn, employing the representation (23)-(24) then gives for (27) in the case of multipole photons the following equation:

$$
W^{(\text {multi) }}(\vec{r})=k^{2} \sum_{\lambda=E, M} \sum_{\mu} \sum_{k, j, m}\left|V_{\lambda k j m \mu}(\vec{r})\right|^{2}
$$

It is seen from the definition of the mode functions (18) and (19) that, in contrast to (28), the zero-point oscillations of the electric field strength of multipole photons manifest the spatial inhomogeneity.

For simplicity, we can compare the monochromatic contributions into (28) and (29) at the same $k$ and $V$. Then

$$
W_{k}^{(\text {plane })}=\sum_{\sigma=1,2} \frac{\hbar k c}{2 V}
$$

and

$$
\begin{aligned}
W_{k}^{(\text {multi })}(\vec{r})= & \sum_{j m \mu} \frac{2 \pi \hbar k c}{V} \\
& \times\left[\mid \sqrt{\frac{j}{2 j+1}} f_{j+1}(k r)\langle 1, j+1, \mu, m-\mu \mid 1 m\rangle Y_{j+1, m-\mu}\right. \\
& -\left.\sqrt{\frac{j+1}{2 j+1}} f_{j-1}(k r)\langle 1, j-1, \mu, m-\mu \mid 1 m\rangle Y_{j-1, m-\mu}\right|^{2} \\
& \left.+\left|f_{j}(k r)\langle 1, j, \mu, m-\mu \mid 1 m\rangle Y_{j, m-\mu}\right|^{2}\right]
\end{aligned}
$$

Since $Y_{\ell, m-\mu} \sim e^{i(m-\mu) \phi}$, this form is independent of the azimuthal angle $\phi$. Moreover, it is a straightforward matter to arrive at the conclusion that

$$
W_{k}^{(\text {multi) }}(\vec{r})=W_{k}^{(\text {multi) }}(r)
$$

(see discussion in Section V.C). 
Consider first the case of standing spherical waves in an ideal spherical cavity when, according to (19)

$$
f_{\ell}(k r)=\sqrt{\frac{\pi}{2 k r}} J_{\ell+1 / 2}(k r)
$$

We stress here that, in the quantum theory of radiation, exactly the standing spherical waves are usually considered [2,27]. Unlike the outgoing and incoming spherical waves of photons, this choice does not lead to the divergence of the vector potential at $r \rightarrow 0$. Taking into account the properties of Bessel functions $J_{\jmath}(x)$, it is easily seen that the principal contribution into $W^{(\text {multi) }}$ in vicinity of the singular point (atom) comes from $J_{1 / 2}(k r)$, corresponding to the electric dipole radiation. The radial dependence of $W^{(\text {multi) }}$ at fixed $k$ and $j=1$ is shown in Fig. 1. It is seen that the vacuum fluctuations are concentrated near atoms where their level can strongly exceed that calculated within the framework of the model of plane waves.

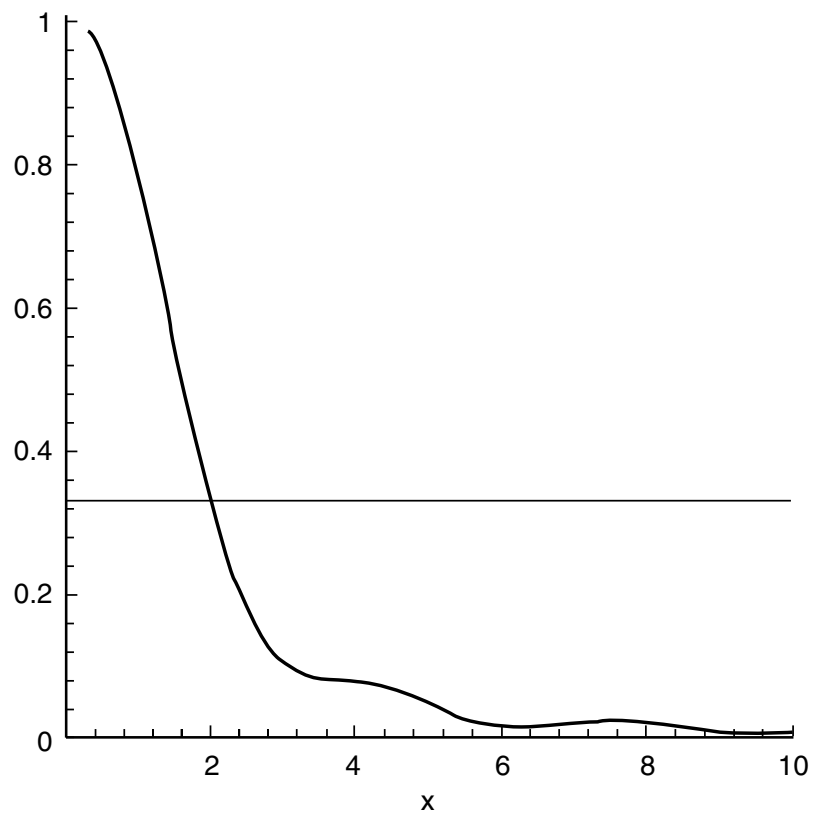

Figure 1. Contribution into the zero-point oscillations (29) from the terms with $j=1$ in the case of an ideal spherical cavity as a function of $x=k r$; (b) the level (28) is shown by the straight line. 


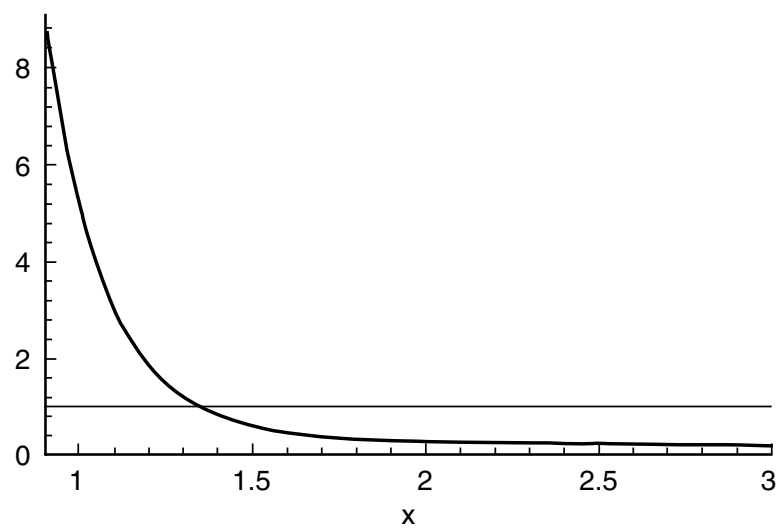

Figure 2. Contribution into (29) from the term $j=1$ for outgoing spherical waves outside the atom with radius $r_{a}=1$ in arbitrary units. The straight line shows the level of $W_{k}^{(\text {plane })}$.

The case of outgoing and incoming spherical waves can be examined under the standard assumption that the atom located at the origin has a finite size that permits us to avoid the divergence at $k r \rightarrow 0$. It is seen that, in some small vicinity of the atom, the zero-point oscillations, corresponding to the multipole field in an infinite space, strongly exceed those in the ideal spherical cavity (see Fig. 2).

It should be stressed that the preceding results were obtained under the assumption that the atom exists only at the origin, no matter whether we use it as an emitter or absorber of radiation. In other words, the spatial inhomogeneity of the zero-point oscillations in (29) reflects the existence of the singular point that, in fact, is the boundary condition for the homogeneous Helmholtz wave equation (5). It is possible to say that the electromagnetic vacuum state "feels" the presence of an atom at the origin and is ready to support any radiation (with all possible $\lambda, j$, and $m$ ) either outgoing or incoming. This is not an astonishing result. The influence of the electromagnetic vacuum state by the presence of an atom has been discussed in quantum electrodynamics for a long time [7,30,31]. The new result here is that the zero-point oscillations are concentrated in some vicinity of atoms where their levels can exceed the standard level (28), which is usually considered.

The point is that the zero-point oscillations are responsible for the so-called shot noise $[14,15]$, determining the quantum limit of uncertainty in different optical measurements. The preceding result shows that the presence of an atom causes the increase of shot noise and hence a deterioration of the quantum limit of precision of measurements, at least, in some vicinity of the atom [22,29]. We discuss this effect in more details in Section VI. 


\section{Summary}

1. Although the monochromatic plane waves of photons are described by only two quantum numbers, specifying the polarization, the monochromatic multipole waves of photons have much more quantum degrees of freedom: the type of radiation (parity) $\lambda=E, M$ and the angular momentum $j \geq 1$ and its projection $m=-j, \ldots, j$.

2. The zero-point oscillations of the energy density of plane waves of photons have the same magnitude everywhere. In contrast, those calculated in the presence of a singular point (source or absorber) manifest spatial inhomogeneity. Precisely, the vacuum noise is concentrated in some vicinity of the singular point.

\section{ATOM-FIELD INTERACTION}

"Well! I've often seen a cat without a grin", thought Alice; "but a grin without a cat! It's the most curious thing I ever saw in all my life".

-Lewis Carroll, Alice's Adventures in Wonderland

\section{A. Multipole Jaynes-Cummings Model}

In the previous section, the classical and quantum electromagnetic fields were considered as absolutely free sourceless objects. This picture follows from the existence of nontrivial solutions of the homogeneous Helmholtz wave equation (5). In some textbooks, this mathematical fact is interpreted as the claim of the following type: the electromagnetic field can exist in the absence of any charge (e.g., see Ref. 60). At the same time, as we know, no one has ever observed photons that had not been created by a source. According to the quantum picture, the electromagnetic vacuum state contains unborn photons of all possible types. They are extracted from this state in the form of overvacuum excitations (waves) in the process of source-photon interaction, leading to the photon generation. The observable properties of real photons are governed by this interaction, which causes the success of conventional $[23,61]$ and correlation $[62,63]$ spectroscopy. Rephrasing Lewis Carroll's Alice's Adventures in Wonderland, it is possible to say that the source is similar to Cheshire Cat, creating grin which propagates in spacetime (see Fig. 3).

The simplest quantum source of photons is the atomic transition, creating, according to the selection rules, multipole photons. The simplest model of the interaction of an atom with the electromagnetic radiation is associated with the notion of so-called two-level atom [64]. In fact, this model originates from the famous study of radiation kinetics by Einstein [65]. With the development of laser, the notion of two-level atom entered firmly into the practice of quantum optics. The fact is that, using lasers as sources of electromagnetic radiation, one 

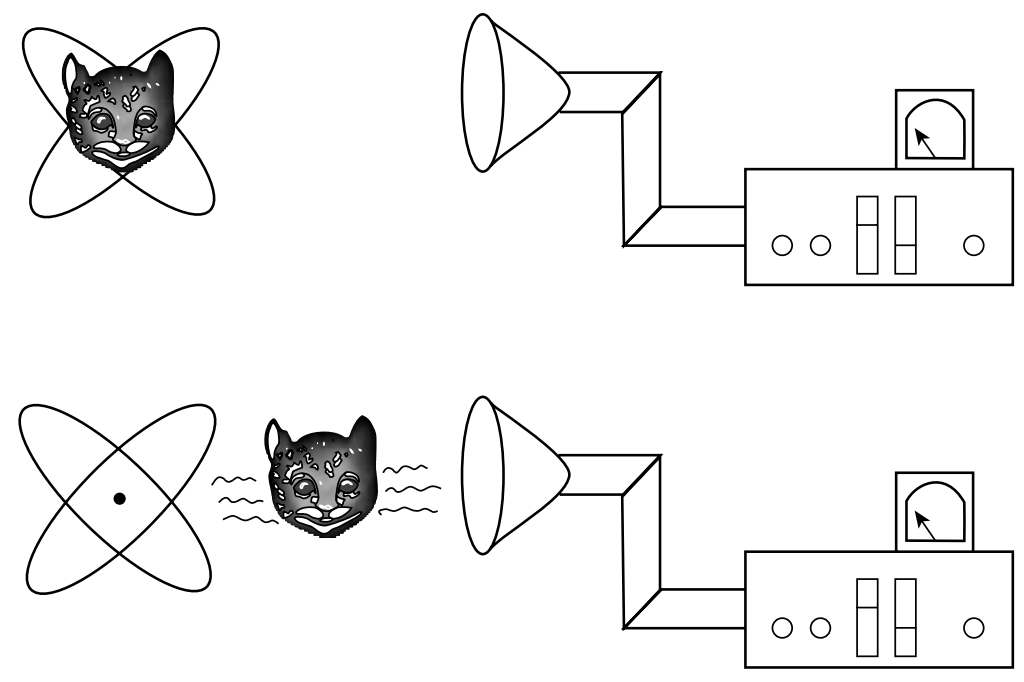

Figure 3. Transmission of information from atom to detector as propagation of the grin of a Cheshire cat. In the upper picture, the atom keeps information about excited level (cat's grin). In the lower picture, the atom hands this information to a photon, propagating to a detector.

can act on the atom with field having frequency very close to the transition frequency between any pair of levels. In this case, the influence of the other levels can be ignored, and one need the consider only a two-level atom (in general, an atom with a finite number of levels) [64]. On the other hand, the use of high-quality cavities has the consequence that an atom in such a cavity interacts with only one or very few modes of the field quantized in the volume of the cavity $[32,33,66]$.

The branch of quantum optics studying the processes of interaction of one or a few atoms with the quantized cavity modes is usually called cavity quantum electrodynamics (cavity QED). The theoretical concepts of cavity QED are based in the first place on investigation of the Jaynes-Cummings model [67] and its generalizations (for a review, see Ref. 68). The reason for this is that the model describes fairly well the physical processes under consideration and at the same time admits an exact solution.

In the usual formulation of the Jaynes-Cummings model, the atom is considered as though it consisted of two nondegenerated levels [67] . In contrast, the radiative transitions in real atoms occur between the states with given angular quantum numbers $|j, m\rangle \rightarrow\left|j^{\prime}, m^{\prime}\right\rangle$ such that $j>j^{\prime} \geq 0$ $[23,26,61]$. This means that, at least the upper level, is degenerated with respect to the quantum number $m(-j \leq m \leq j)$. For example, in the simplest case of the electric dipole transition between the states $|j=1, m=0, \pm 1\rangle$ and 


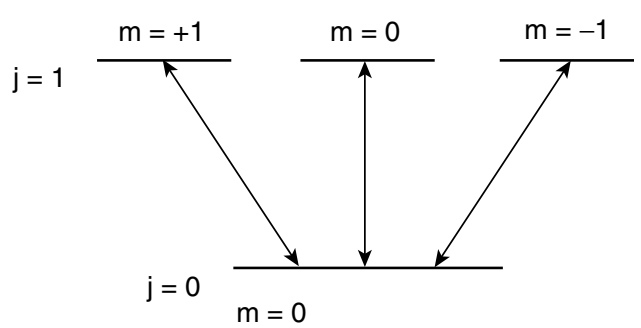

Figure 4. Scheme of transitions between the triple degenerated excited state $|j=1, m=0, \pm 1\rangle$ and ground state $\left|j^{\prime}=0, m=0\right\rangle$ in a two-level atom with the dipole transition.

$\left|j^{\prime}=0, m^{\prime}=0\right\rangle$, the excited state is triple degenerated (see Fig. 4). The corresponding generalization of the Jaynes-Cummings model has been discussed [36,53]. Similar models have been considered in different problems of interaction of quantum light and matter [69].

Interaction between a single atom and a radiation field is usually considered within the framework of perturbation theory using the following Hamiltonian $[26,64]$

$$
H=H_{0}+\frac{1}{2 m_{e}}\left(\vec{p}-\frac{e}{c} \vec{A}\right)^{2}
$$

where $H_{0}$ describes the unperturbed atom and field and the rest is written for the interaction between a single spinless electron with charge $e$, mass $m_{e}$, momentum $p$, and free electromagnetic field described by the vector potential $\vec{A}$. Following earlier observations [36,53], consider a two-level atom with the electric dipole transition between the triple-degenerated excited atomic state with $j=1$ and nondegenerated ground state with $j^{\prime}=0$. The atom is supposed to be located at the center of an ideal spherical cavity. The coupling constant of the atom-field interaction can be found by calculating the matrix element $[26,27]$

$$
-\frac{e}{2 m_{e} c}\langle 0,0|\vec{p} \cdot \vec{A}+\vec{A} \cdot \vec{p}| 1, m\rangle=i k_{0}\langle 0,0|\vec{d} \cdot \vec{A}| 1, m\rangle
$$

obtained from (30). The $A^{2}$ term is excluded because of the use of the so-called rotating-wave approximation [64]. Here $\vec{d}=e \vec{r}$ is the dipole moment and $\vec{A}(\vec{r})$ is the operator vector potential (24) with the radial dependence of the mode functions (18) described by $f_{\ell}(k r)=j_{\ell}(k r)$ in (19) due to the choice of the boundary conditions.

Assuming the central symmetry of atomic field and taking into account the fact that the spin state of an atom does not change under the electric dipole 
transition $[27,61]$, we can represent the atomic states in (31) as

$$
|1, m\rangle=\mathscr{R}_{1}(k r) Y_{1 m}(\theta, \phi), \quad|0,0\rangle=\mathscr{R}_{0}(k r) Y_{00}(\theta, \phi)
$$

where $\mathscr{R}_{\ell}$ is the radial part of the atomic wave function. Then, representing the dipole moment $\vec{d}$ in the helicity basis (16) and carrying out the calculations of integrals in (31) over the atomic volume, we get

$$
\forall m \quad g \equiv k_{0}\langle 1, m|\vec{d} \cdot \vec{A}| 0,0\rangle=k_{0} D \sqrt{\frac{3 \hbar c}{10 k R}}
$$

where

$$
D=e \int_{0}^{r_{a}} d r r^{3} f_{1}(k r) f_{0}(k r)
$$

is the effective dipole factor. Here $R$ and $r_{a}$ denote the cavity and atomic radii respectively and $k$ is the wave number, describing the cavity field.

Taking into account the explicit form of spherical Bessel functions [70]

$$
\begin{aligned}
& j_{0}(k r)=\frac{\sin k r}{k r}, \\
& j_{2}(k r)=\frac{3-(k r)^{2}}{(k r)^{3}} \sin k r-\frac{3 \cos k r}{(k r)^{2}}
\end{aligned}
$$

we note that, owing to the structure of the mode functions (18), all other radial functions do not contribute (24). Assuming that the atom is a point-like object (in fact, very small with respect to the wavelength of radiation field), we get

$$
\lim _{k r \rightarrow 0} j_{0}(k r)=1, \quad \lim _{k r \rightarrow 0} j_{2}(k r)=0
$$

Using the properties of the Clebsch-Gordon coefficients [71] and spherical harmonics [70], for the mode functions (18) in this limit we get

$$
V_{E k 1 m}(0) \sim-\delta_{m \mu}
$$

Inserting this into (24), we obtain

$$
\vec{A}_{E k 1}(0)=-\sqrt{\frac{\hbar c}{3 k V}} \sum_{m=-1}^{1}(-1)^{\mu} \vec{\chi}_{-\mu} a_{E k 1 m} \delta_{m \mu}
$$

This means that the electric dipole transition $|1, m\rangle \rightarrow|0,0\rangle$ creates a photon with spin state (polarization) $\mu=m$. However, the picture of the polarization 
changes with the distance from the atom because of the position dependence of the mode functions (18).

Thus, the Jaynes-Cummings Hamiltonian for the electric dipole transition can be written as follows [36,53]:

$$
\begin{aligned}
H & =H_{0}+H_{\mathrm{int}} \\
H_{0} & =\sum_{m=-1}^{1}\left\{\omega a_{E 1 m}^{+} a_{E 1 m}+\omega_{0} R_{m m}\right\} \\
H_{\mathrm{int}} & =g \sum_{m=-1}^{1}\left\{R_{m g} a_{E 1 m}+a_{E 1 m}^{+} R_{g m}\right\}
\end{aligned}
$$

Here $\omega_{0}=\hbar k_{0} c$ and $\omega=\hbar k c$ are the energies of the atomic transition and cavity field, respectively, and the atomic operators are defined as follows:

$$
R_{m g}=|1, m\rangle\left\langle 0,0\left|, \quad R_{m m^{\prime}}=\right| 1, m\right\rangle\left\langle 1, m^{\prime}\right|
$$

The first term in (34) describes the energy of the free cavity field and atom, while the second term gives the energy either of the transition $|1, m\rangle \rightarrow|0,0\rangle$ with generation of the multipole photon or of the transition $|0,0\rangle \rightarrow|1, m\rangle$ accompanied by the absorption of corresponding electric dipole photon.

Generalizations of the Jaynes-Cummings model (34) in the case of quadrupole and other high-order multipole transitions can be constructed in the same way.

\section{B. The $S U(2)$ Atomic Phase States}

In the model Hamiltonian (34), the excited atomic state is specified by the following three orthogonal states:

$$
|1,1\rangle, \quad|1,0\rangle, \quad|1,-1\rangle
$$

On this basis, we can construct a representation of the $S U(2)$ algebra with the following generators $[53,54]$

$$
\begin{aligned}
J_{z} & =R_{++}-R_{--} \\
J_{+} & =\sqrt{2}\left(R_{+0}+R_{0-}\right) \\
J_{-} & =\sqrt{2}\left(R_{0+}+R_{-}\right)
\end{aligned}
$$

which obey the standard commutation relations

$$
\left[J_{+}, J_{-}\right]=2 J_{z}, \quad\left[J_{z}, J_{ \pm}\right]= \pm J_{ \pm}
$$


The enveloping algebra of (37)-(38) contains the uniquely defined Casimir operator

$$
\mathbf{J}^{2}=2 \sum_{m=-1}^{1} R_{m m} \equiv 2 \times \mathbf{1}
$$

where

$$
\mathbf{1}=|1,1\rangle\langle 1,1|+| 1,0\rangle\langle 1,0|+| 1,-1\rangle\langle 1,-1|
$$

is the unit operator in the space spanned by the basis (36).

The existence of (39) permits us to use the method, proposed by Vourdas [51], to construct the dual representation of the $S U(2)$ algebra (37)-(38). Following [51], we represent the lowering and rising operators in (37) as

$$
J_{+}=J_{r} \varepsilon, \quad J_{-}=\varepsilon^{+} J_{r}
$$

where $J_{r}$ is the Hermitian "radial" operator and $\epsilon$ is the unitary

$$
\varepsilon \varepsilon^{+}=\mathbf{1}
$$

"exponential of the phase" operator. The terminology here is borrowed from complex calculus. It is clear that the phase variable here describes the azimuth of the angular momentum of the excited atomic state. Equations (40) can now be done in a straightforward manner to yield

$$
\begin{aligned}
J_{r} & =\sqrt{2}\left(\mathbf{1}-R_{--}\right) \\
\varepsilon & =R_{+0}+R_{0-}+e^{i \psi} R_{-+}
\end{aligned}
$$

where $\psi$ is an arbitrary real parameter describing the so-called atomic reference phase [53,54]. It is clear that $\epsilon$ is a Coxeter-type operator [72] because

$$
\varepsilon^{3}=e^{i \psi} \times \mathbf{1}
$$

In analogy to complex calculus, we can now define the cosine and sine of the atomic $S U(2)$ phase operators [36]

$$
C_{a}=\frac{\varepsilon+\varepsilon^{+}}{2}, \quad S_{a}=\frac{\varepsilon-\varepsilon^{+}}{2 i}
$$

such that

$$
C_{a}^{2}+S_{a}^{2}=\mathbf{1}
$$


and

$$
\left[C_{a}, S_{a}\right]=0
$$

Following [51], we now introduce the dual representation of the $S U(2)$ algebra (37)-(38). Consider first the eigenstates of the exponential operator in (41):

$$
\varepsilon\left|\phi_{m}\right\rangle=e^{i \phi_{m}}\left|\phi_{m}\right\rangle
$$

It is a straightforward matter to arrive at the relations [46]

$$
\left|\phi_{m}\right\rangle=\frac{1}{\sqrt{3}} \sum_{m^{\prime}=-1}^{1} e^{-i m^{\prime} \phi_{m}}|1, m\rangle, \quad \phi_{m}=\frac{2 m \pi-\psi}{3}
$$

where $m$ acquires the values 0 and \pm 1 as above. It is easily seen that the socalled phase states [51] (45) determine the basis dual to (36) [46]. In particular

$$
\sum_{m=-1}^{1}\left|\phi_{m}\right\rangle\left\langle\phi_{m}\right|=\mathbf{1}
$$

Then, the atomic $S U(2)$ quantum phase operator can be defined as follows:

$$
\hat{\phi}=\sum_{m=-1}^{1} \phi_{m}\left|\phi_{m}\right\rangle\left\langle\phi_{m}\right|=-\frac{\psi}{3} \mathbf{1}-\frac{2 i \pi}{3 \sqrt{3}}\left(e^{-i \psi / 3} \epsilon-e^{i \psi / 3} \epsilon^{+}\right)
$$

In turn, the cosine and sine operators (42) can be represented as the functions

$$
C_{a}=\cos \hat{\phi}, \quad S_{a}=\sin \hat{\phi}
$$

of the operator (47). Then, the dual representation of the atomic $S U(2)$ algebra (37) is provided by the following generators:

$$
\begin{aligned}
& J_{z}^{(\phi)}=\sum_{m} m\left|\phi_{m}\right\rangle\left\langle\phi_{m}\right| \\
& J_{+}^{(\phi)}=\sum_{m} \sqrt{2-m(m+1)}\left|\phi_{m+1}\right\rangle\left\langle\phi_{m}\right| \\
& J_{-}^{(\phi)}=\sum_{m} \sqrt{2-m(m-1)}\left|\phi_{m-1}\right\rangle\left\langle\phi_{m}\right|
\end{aligned}
$$

Similar results can be obtained for an arbitrary atomic multipole transition in much the same way as above. For example, in the case of the excited atomic 
state with $j=2$, the representation of the $S U(2)$ algebra takes the form

$$
\begin{aligned}
& J_{+}=\sqrt{2}|2\rangle\langle 1|+\sqrt{3}| 1\rangle\langle 0|+\sqrt{3}| 0\rangle\langle-1|+\sqrt{2}|-1\rangle\langle-2| \\
& J_{-}=\sqrt{2}|1\rangle\langle 2|+\sqrt{3}| 0\rangle\langle 1|+\sqrt{3}|-1\rangle\langle 0|+\sqrt{2}|-2\rangle\langle-1| \\
& J_{z}=2|2\rangle\langle 2|+| 1\rangle\langle 1|-|-1\rangle\langle-1|-2|-2\rangle\langle-2|
\end{aligned}
$$

where $|n\rangle \equiv|m=n\rangle$. The corresponding exponential of the phase operator is

$$
\varepsilon=|2\rangle\langle 1|+| 1\rangle\langle 0|+| 0\rangle\langle-1|+|-1\rangle\left\langle-2\left|+e^{i \psi}\right|-2\right\rangle\langle 2|
$$

In this case, the eigenvalues of the phase variable take the following five independent values:

$$
\phi_{m}=\frac{\psi+2 m \pi}{5}, \quad m=2,1, \ldots,-2
$$

In the general case of an arbitrary integer $j \geq 1$, the number of independent eigenvalues of the phase variable $\phi_{m}$ is $(2 j+1)$.

\section{EPR Paradox and Entanglement}

The preceding formalism of $S U(2)$ phase states can be used in a number of problems of quantum physics. As an illustrative example of great importance, consider the so-called Einstein-Podolsky-Rosen (EPR) paradox [73] (see also discussions in Refs. 14, 15, 74, and 75). The EPR paradox touches on the conceptual problems of reality and locality and existence of hidden variables in quantum physics as well as the more technological aspects of quantum cryptography [34].

In the original EPR gedanken experiment [73], a two-component system, consisting of two spin $-\frac{1}{2}$ particles, is considered. Up to some time $t_{0}$, these particles are taken to be in a bounded state of zero angular momentum. At $t_{0}$, the binding is taken off without any disturbance of the spin states. Then, the separated particles move off in the opposite directions. Since the particles are in the common quantum state, the measurement of one chosen variable of particle 1 , moving "to the left," completely determines the outcome of a measurement of corresponding variable of particle 2, moving "to the right."

Before making the measurement, the system is supposed to be in the EPR state, which is also called the entangled state. It is described by the wavefunction of the form

$$
\left|\Psi_{ \pm}^{(\mathrm{EPR})}\right\rangle=\frac{1}{\sqrt{2}}\left(\left|\uparrow_{L} \downarrow_{R}\right\rangle \pm\left|\downarrow_{L} \uparrow_{R}\right\rangle\right)
$$


Here $\left|\uparrow_{L, R}\right\rangle$ denotes the spinup or spindown state of the left or right particle. The two state vectors in the right-hand side of (49) form a basis of the corresponding Hilbert space in which we can define the representation of the $S U(2)$ algebra by the following generators

$$
\begin{aligned}
J_{+} & =\left|\uparrow_{L} \downarrow_{R}\right\rangle\left\langle\downarrow_{L} \uparrow_{R}\right| \\
J_{-} & =\left|\downarrow_{L} \uparrow_{R}\right\rangle\left\langle\uparrow_{L} \downarrow_{R}\right| \\
J_{z} & =\frac{1}{2}\left(\left|\uparrow_{L} \downarrow_{R}\right\rangle\left\langle\uparrow_{L} \downarrow_{R}|-| \downarrow_{L} \uparrow_{R}\right\rangle\left\langle\downarrow_{L} \uparrow_{R}\right|\right)
\end{aligned}
$$

so that these operators obey the commutation relations (38) as well as the condition (39). Hence, the operators (47) admit a polar decomposition of the form discussed in the previous subsection. In particular, the exponential of the $S U(2)$ phase operator (41) takes the form

$$
\varepsilon=\left|\uparrow_{L} \downarrow_{R}\right\rangle\left\langle\downarrow_{L} \uparrow_{R}\left|+e^{i \psi}\right| \downarrow_{L} \uparrow_{R}\right\rangle\left\langle\uparrow_{L} \downarrow_{R}\right|
$$

where $\psi$ is again an arbitrary real reference phase. The $S U(2)$ phase states of the type of (45), and (46) which are defined to be the eigenstates of the operator (51), have the form

$$
\left|\phi_{\sigma}\right\rangle=\frac{1}{\sqrt{2}}\left(\left|\uparrow_{L} \downarrow_{R}\right\rangle+e^{i \phi_{\sigma}}\left|\downarrow_{L} \uparrow_{R}\right\rangle\right)
$$

where

$$
\phi_{\sigma}=\frac{\psi}{2}+\sigma \pi, \quad \sigma=0,1
$$

It is now easily seen that, at $\psi=0$, the $S U(2)$ phase states (52) coincide with the EPR states (49). Thus, the EPR states can be interpreted as the eigenstates of the exponential of the phase operator (51) of the $S U(2)$ algebra (50). The corresponding quantum phase operator takes the form

$$
\hat{\phi}=\frac{\psi+\pi}{2} \times \mathbf{1}-\frac{\pi}{2} e^{-i \psi / 2} \varepsilon
$$

where

$$
\mathbf{1} \equiv\left|\uparrow_{L} \downarrow_{R}\right\rangle\left\langle\uparrow_{L} \downarrow_{R}|+| \downarrow_{L} \uparrow_{R}\right\rangle\left\langle\downarrow_{L} \uparrow_{R}\right|
$$

By construction, the operator (53) describes the relative phase between the two EPR states (49).

There are many different physical realizations of the EPR or entangled states in optics and condensed-matter physics. For example, the creation of two photons with different helicities by a single atom in the process of cascade 
decay of transition of the type $j-j^{\prime}=0$ leads to the polarization-entangled state of the photons, leaving the atom in opposite directions (e.g., see Section 12.14.1 in Ref. 14).

Another important example of entanglement is provided by the system of two 2-level atoms in an optical resonator [76]. Such a system can be described by the following Jaynes-Cummings Hamiltonian:

$$
\begin{aligned}
H & =H_{0}+H_{\mathrm{int}} \\
H_{0} & =\sum_{f=1,2} \omega_{f}\left|e_{f}\right\rangle\left\langle e_{f}\right|+\omega a^{+} a \\
H_{\mathrm{int}} & =\sum_{f=1,2} i \gamma_{f}\left(\left|e_{f}\right\rangle\left\langle g_{f}\left|a-a^{+}\right| g_{f}\right\rangle\left\langle e_{f}\right|\right)
\end{aligned}
$$

Here index $f$ denotes the atom in the cavity, $\left|e_{f}\right\rangle\left(\left|g_{f}\right\rangle\right)$ is the excited (ground) state of the corresponding atom, $\gamma_{f}$ is the atom-field coupling constant, and the operators $a^{+}$and $a$ describe the cavity photons. Among the eigenstates of (54)

$$
\begin{aligned}
\left|\psi_{0}\right\rangle & =\frac{1}{\sqrt{\gamma_{1}^{2}+\gamma_{2}^{2}}}\left(\gamma_{1}\left|0 ; g_{1} ; e_{f}\right\rangle-\gamma_{2}\left|0 ; e_{1} ; g_{2}\right\rangle\right) \\
\left|\psi_{ \pm}\right\rangle & =\frac{1}{\sqrt{2}}\left(\left|1 ; g_{1} ; g_{2}\right\rangle \pm \frac{i}{\sqrt{\gamma_{1}^{2}+\gamma_{2}^{2}}}\left(\gamma_{2}\left|0 ; g_{1} ; e_{2}\right\rangle+\gamma_{1}\left|0 ; e_{1} ; g_{2}\right\rangle\right)\right)
\end{aligned}
$$

there is a maximally entangled atomic state $\left|\psi_{0}\right\rangle$, which, under the assumption that $\gamma_{1}=\gamma_{2}$, takes the form

$$
\begin{aligned}
\left|\psi_{0}\right\rangle & =|0\rangle \otimes\left|\psi_{\mathrm{ent}}\right\rangle \\
\left|\psi_{\mathrm{ent}}\right\rangle & =\frac{1}{\sqrt{2}}\left(\left|g_{1} ; e_{2}\right\rangle-\left|e_{1} ; g_{2}\right\rangle\right)
\end{aligned}
$$

similar to EPR state (49). In the above formulas we use the following notations:

$$
\begin{aligned}
\left|0 ; e_{f} ; g_{f^{\prime} \neq f}\right\rangle & =|0\rangle_{\text {field }} \otimes\left|e_{f}\right\rangle \otimes\left|g_{f^{\prime}}\right\rangle \\
\left|1 ; g_{1} ; g_{2}\right\rangle & =|1\rangle_{\text {field }} \otimes\left|g_{1}\right\rangle \otimes\left|g_{2}\right\rangle
\end{aligned}
$$

To establish contact with the $S U(2)$ phase states, we can consider the following representation of generators of the atomic $S U(2)$ algebra

$$
\begin{aligned}
& J_{+}=\left|e_{1}\right\rangle\left\langle e_{2}\right| \\
& J_{-}=\left|e_{2}\right\rangle\left\langle e_{1}\right| \\
& J_{z}=\frac{1}{2}\left(\left|e_{1}\right\rangle\left\langle e_{1}|-| e_{2}\right\rangle\left\langle e_{2}\right|\right.
\end{aligned}
$$


similar to (50). Then, the exponential of the phase operator takes the form (for simplicity, we put here $\psi=0$ )

$$
\varepsilon=\left|e_{1}\right\rangle\left\langle e_{2}|+| e_{2}\right\rangle\left\langle e_{1}\right|
$$

It is now seen that the maximally entangled atomic state in (55) is again the $S U(2)$ quantum phase state.

An interesting example of entanglement in condensed matter is represented by the formation of Cooper pairs in conventional superconductors. It is well known that the electron-phonon interaction in metals can lead to formation of collective quantum states of paired electrons with opposite spins and linear momenta [77]. In the simplest quasispin form, the system can be specified by the Hamiltonian [78,79]

$$
H=\sum_{p} E_{p} \sigma_{p}^{z}-\sum_{p, p^{\prime}} J_{p p^{\prime}} \sigma_{p}^{-} \sigma_{p^{\prime}}^{+}
$$

where $E_{p}$ denotes the energy spectrum depending on the momentum $p$ of electrons, $J_{p p^{\prime}}$ is the effective coupling constant, and the Pauli operators

$$
\sigma_{p}^{-}=\left(\begin{array}{cc}
0 & 1 \\
0 & 0
\end{array}\right), \quad \sigma_{p}^{+}=\left(\begin{array}{cc}
0 & 0 \\
1 & 0
\end{array}\right), \quad \sigma_{p}^{z}=\frac{1}{2}\left(\begin{array}{cc}
1 & 0 \\
0 & -1
\end{array}\right)
$$

correspond to the pairs of electrons with opposite spins and momenta. Since the Pauli operators obey the commutation relations

$$
\left[\sigma_{p}^{-}, \sigma_{p^{\prime}}^{+}\right]=2 \sigma_{p}^{z} \delta_{p p^{\prime}}, \quad\left[\sigma_{p}^{z}, \sigma_{p^{\prime}}^{\mp}\right]= \pm \sigma_{p}^{\mp} \delta_{p p^{\prime}}
$$

which coincide with (38); by performing an analysis similar to that described in previous subsection, we get

$$
\varepsilon_{p}=\sigma_{p}^{-}+e^{i \psi} \sigma_{p}^{+}
$$

This is the $S U(2)$ exponential of the phase operator similar to (41) defined for each $p$.

It is also known that, in the so-called thermodynamic limit, when the number of electrons tends to infinity at constant density, the state of the system with the quasispin Bardeen-Cooper-Shriefer (BCS) Hamiltonian (57) is the eigenstate of the trial Hamiltonian, in which the interaction part of (57) is changed by the operator [79]

$$
H_{\mathrm{int}}=-T_{c} \sum_{p}\left(\sigma_{p}^{-} \Delta+\sigma_{p}^{+} \Delta^{*}-|\Delta|^{2}\right)
$$


where $T_{c}$ is some constant related to $J_{p p^{\prime}}$ in (57) and $\Delta$ is the complex parameter, characterizing the gap in the spectrum of eigenenergy and depending on the temperature. Thus, at $\psi=-2 \arg \Delta$, the superconducting state (the eigenstate of $\varepsilon_{p}$ ) is the $S U(2)$ quantum phase state, describing entangled electrons with opposite spins and momenta (Cooper pair). Therefore, the phase transition into the superconducting state can be interpreted as the creation of collective entangled state of electrons.

\section{Summary}

1. For the electric dipole radiation described by the Jaynes-Cummings Hamiltonian (34), the polarization of photons at $k r \rightarrow 0$ is defined by the quantum number $m=0, \pm 1$, describing the excited atomic state.

2. For any atomic multipole transition, the excited state can be described in terms of the dual representation of corresponding $S U(2)$ algebra, describing the azimuthal quantum phase of the angular momentum. In particular, the exponential of the phase operator and phase states can be constructed. The quantum phase variable has a discrete spectrum with $(2 j+1)$ different eigenvalues.

3. In a special case of $j=\frac{1}{2}$, the eigenstates of the exponential of the phase operator coincide with the EPR (entangled) states, which can be interpreted as the $S U(2)$ phase state.

\section{QUANTUM PHASE OF MULTIPOLE RADIATION}

No, my dear Watson, the two events are connected - must be connected. It is for us to find the connection.

— Sir Arthur Conan Doyle, The Second Stain

\section{A. Conservation of Angular Momentum in the Process of Radiation}

We now turn to the problem of the $S U(2)$ quantum phase of multipole radiation. As a particular example of some considerable interest, we investigate the electric dipole field. All other types of the multipole radiation can be considered in the same way.

In Section III.B, we introduced the atomic quantum phase states through the use of the representation of the $S U(2)$ algebra (37) and dual representation (48), corresponding to the angular momentum of the excited atomic state. The multipole radiation emitted by atoms carries the angular momentum of the excited atomic state and can also be specified by the angular momentum $[2,26,27]$. The bare operators of the angular momentum of the electric dipole 
radiation have the form

$$
\begin{aligned}
& M_{+}=\sqrt{2}\left(a_{+}^{+} a_{0}+a_{0}^{+} a_{-}\right) \\
& M_{-}=\sqrt{2}\left(a_{0}^{+} a_{+}+a_{-}^{+} a_{0}\right) \\
& M_{z}=\sum_{m=-1}^{1} m a_{m}^{+} a_{m}
\end{aligned}
$$

This result can be obtained by canonical quantization of the components of classical angular momentum (9) [2]. Hereafter in this section we use the following notation:

$$
a_{m} \equiv a_{E k 1 m}
$$

Taking into account the commutation relations (23), it is easy to check that

$$
\left[M_{+}, M_{-}\right]=2 M_{z}, \quad\left[M_{z}, M_{ \pm}\right]= \pm M_{ \pm}
$$

so that the operators (58) form a representation of the $S U(2)$ subalgebra in the Weyl-Heisenberg algebra (18) of the electric dipole photons.

The electric dipole photons, as well as the operators (58), are defined in the Hilbert space

$$
\mathscr{H}_{\text {field }}=\bigotimes_{m=-1}^{1} \mathscr{H}_{m}
$$

where each subspace $\mathscr{H}_{m}$ is spanned by the countable set of Fock vectors $\left|n_{m}\right\rangle$ $\left(n_{m}=0,1,2, \ldots\right)$, which obey the orthogonality condition

$$
\left\langle n_{m^{\prime}}^{\prime} \mid n_{m}\right\rangle=\delta_{n n^{\prime}} \delta_{m m^{\prime}}
$$

and the completeness condition

$$
\bigotimes_{m=-1}^{1} \sum_{n_{m}=0}^{\infty}\left|n_{m}\right\rangle\left\langle n_{m}\right|=\mathbf{1}
$$

Here $\mathbf{1}$ is the unit operator, acting in (60). Unlike (39)

$$
M^{2}=M_{z}^{2}+M_{+} M_{-}-M_{z} \neq \mathbf{1}
$$

in the whole Hilbert space (60). In other words, there is no isotype representation [52] of (58) in (60). Therefore, the polar decomposition of the 
$S U(2)$ subalgebra (58) in the Weyl-Heisenberg algebra of electric dipole photons cannot be constructed in the way discussed in Section III.B.

At the same time, we know that the photons carry information obtained from the atom in the process of generation. This information is transmitted through the conservation laws. In particular, the photon carries the angular momentum of the excited state because

$$
\left[\left(J_{\alpha}+M_{\alpha}, H\right]=0\right.
$$

Here $J_{\alpha}$ denotes the atomic $S U(2)$ generators (37) with $\alpha=z, \pm, M_{\alpha}$ is the component of the field angular momentum operator (58), and $H$ is the JaynesCummings model Hamiltonian (34).

Since the atomic $S U(2)$ quantum phase, discussed in Section III.B, is defined by the angular momentum of the excited atomic state, the conservation law (62) can be used to determine the field counterpart of the exponential of the phase operator (41) and other operators referred to the $S U(2)$ quantum phase [36,46]. For example, it is easily seen that the operator

$$
\varepsilon_{\mathrm{rad}}=a_{+}^{+} a_{0}+a_{0}^{+} a_{-}+a_{-}^{+} a_{+}
$$

complements the atomic exponential of the phase operator (41) (at $\psi=0$ ) with respect to the integral of motion:

$$
\left[\left(\varepsilon_{a}+\varepsilon_{\mathrm{rad}}\right), H\right]=0
$$

The operator (63) can be considered as the result of "mapping" of the atomic exponential of the $S U(2)$ phase operator (41) on the field variables through the use of the integral of motion (64). Unlike (41), it is not unitary

$$
\varepsilon_{\mathrm{rad}} \varepsilon_{\mathrm{rad}}^{+} \neq \mathbf{1}
$$

but it is a normal operator

$$
\left[\varepsilon_{\mathrm{rad}}, \varepsilon_{\mathrm{rad}}^{+}\right]=0
$$

commuting with the total number of photons

$$
\left[\varepsilon_{\mathrm{rad}}, \sum_{m} a_{m}^{+} a_{m}\right]=0
$$

In the same way, it is easy to show that the operator constructions $\varepsilon_{\mathrm{rad}}+\varepsilon_{\mathrm{rad}}^{+}$and $-i\left(\varepsilon_{\mathrm{rad}}-\varepsilon_{\mathrm{rad}}^{+}\right)$complement the atomic cosine and sine of the $S U(2)$ phase operators (42) with respect to the integrals of motion with the atom-field Hamiltonian (34). 


\section{B. Dual Representation of Dipole Photons}

We now turn to the construction of the dual representation of the photon operators, providing the field counterpart of the $S U(2)$ phase representation of the atomic variables. It is easily seen that the atomic exponential of the $S U(2)$ phase operator (41) takes [in the representation of dual states (46)] the following diagonal form

$$
\varepsilon_{a}^{(\phi)}=\sum_{m=-1}^{1} e^{i \phi_{m}}\left|\phi_{m}\right\rangle\left\langle\phi_{m}\right|
$$

where $\phi_{m}$ takes the values (46) (hereafter we put $\psi=0$ without loss of generality). Thus, the dual representation of the atomic operators leads to the diagonal form of the exponential of the phase operator.

In turn, the field operator (63), representing the field counterpart of (41), can be diagonalized by the following Bogolubov-type [80] canonical transformation [46]

$$
\begin{aligned}
& a_{m}=\frac{1}{\sqrt{3}} \sum_{m^{\prime}=-1}^{1} e^{-i m^{\prime} \phi_{m}} \mathbf{a}_{m^{\prime}} \\
& \mathbf{a}_{m}=\frac{1}{\sqrt{3}} \sum_{m^{\prime}=-1}^{1} e^{i m^{\prime} \phi_{m}} a_{m^{\prime}}
\end{aligned}
$$

which has the form of finite Fourier transformation with $\phi_{m}$ defined in (46). It follows from the commutation relations (23) that

$$
\left[\mathbf{a}_{m}, \mathbf{a}_{m^{\prime}}^{+}\right]=\delta_{m m^{\prime}}
$$

Hence, the operators a in (67) also form a representation of the WeylHeisenberg algebra of the electric dipole photons. Employing this transformation (67) then gives the diagonal representation of the operator (63)

$$
\varepsilon_{\mathrm{rad}}^{(\phi)}=\sum_{m=-1}^{1} e^{i \phi_{m}} \mathbf{a}_{m}^{+} \mathbf{a}_{m}
$$

similar to (66). It is now a straightforward matter to arrive at the integral of motion:

$$
\left[\left(\varepsilon_{a}^{(\phi)}+\varepsilon_{\mathrm{rad}}^{(\phi)}\right), H\right]=0
$$

By construction, it corresponds to (64) in the dual representation of the dynamical variables for the atom and radiation field. This integral of motion reflects the fact that the $S U(2)$ phase information is also transmitted from the 
atom to photon in the process of generation. In other words, the integral of motion (70) is responsible for the mapping of the atomic $S U(2)$ phase on the field variables. Therefore, one can choose to interpret $\mathbf{a}_{m}$ and $\mathbf{a}_{m}^{+}$in the canonical transformation (67) as the annihilation and creation operators of the electric dipole photons with given radiation phase [46].

As can be seen from the transformation (67), the operators $\mathbf{a}_{m}$ obey the same stability condition (25) as $a_{m}$

$$
\forall m, m^{\prime} \quad a_{m}|0\rangle=\mathbf{a}_{m^{\prime}}|0\rangle=0
$$

where the dipole vacuum state is defined as follows:

$$
|0\rangle \equiv \bigotimes_{m=-1}^{1}\left|0_{m}\right\rangle
$$

Hence, the creation operators $\mathbf{a}_{m}^{+}$in (67) can be used to generate the Fock number states in the "phase representation"

$$
\left|v_{m}\right\rangle=\frac{1}{\sqrt{v_{m} !}}\left(\mathbf{a}_{m}^{+}\right)^{v_{m}}|0\rangle
$$

such that

$$
\mathbf{a}_{m}^{+} \mathbf{a}_{m}\left|v_{m}\right\rangle=v_{m}\left|v_{m}\right\rangle, \quad v_{m}=0,1, \ldots
$$

and

$$
\left\langle v_{m} \mid v_{m^{\prime}}^{\prime}\right\rangle=\delta_{m m^{\prime}} \delta_{v v^{\prime}}, \quad \bigotimes_{m=-1}^{1} \sum_{v_{m}}\left|v_{m}\right\rangle\left\langle v_{m}\right|=\mathbf{1}
$$

The unit operator here coincides with (61). Thus, the states (72) form a basis in the Hilbert space (60) dual to the basis of conventional number states $\left|n_{m}\right\rangle$. In analogy to the atomic phase states (46), we call (72) the radiation phase states of the electric dipole photons. It follows from (69) that the radiation phase states (72) are the eigenstates of the operator $\varepsilon_{\text {rad }}^{(\phi)}$ :

$$
\varepsilon_{\mathrm{rad}}^{(\phi)}\left|v_{m}\right\rangle=v_{m} e^{i \phi_{m}}\left|v_{m}\right\rangle
$$

In contrast to the relation (45), the eigenvalues of $\varepsilon_{\mathrm{rad}}^{(\phi)}$ in (73) contain, in addition to the exponential, a factor of $v_{m}$, describing the number of photons in a given radiation phase state. Thus, this is an non-normalized exponential of the phase operator. 
The preceding results lead to the conclusion that the radiation phase states (72) are dual to the conventional Fock number states $\left|n_{m}\right\rangle$. In turn, the operators (67) form the representation of the Weyl-Heisenberg algebra of the electric dipole photons dual to the operators $a_{m}$ and $a_{m}^{+}$[46].

Although the canonical transformation (67) has the very simple form of the finite Fourier transformation, the connection between the conventional number states and the radiation phase states (72) is not simple:

$$
\left|v_{m}\right\rangle=\sqrt{\frac{v_{m} !}{3^{v_{m}}}} \sum_{n_{0}=0}^{v_{m}} \sum_{n_{+}=0}^{v_{m}-n_{0}} \frac{\exp \left[\left(i\left(v_{m}-n_{0}-2 n_{+}\right) \phi_{m}\right]\right.}{\sqrt{n_{0} ! n_{+} !\left(v_{m}-n_{0}-n_{+}\right) !}} \times\left|n_{+} ; n_{0} ; v_{m}-n_{0}-n_{+}\right\rangle
$$

It is interesting that the "dual" coherent states

$$
\begin{aligned}
\left|\alpha^{(a)}\right\rangle & =\prod_{m} D_{m}^{(a)}\left(\alpha^{(a)}\right)|0\rangle \\
\left|\alpha^{(\mathbf{a})}\right\rangle & =\prod_{m} D^{(\mathbf{a})}\left(\alpha^{(\mathbf{a})}\right)|0\rangle
\end{aligned}
$$

are equivalent up to the following transformation of the parameters:

$$
\begin{aligned}
\alpha_{m^{\prime}}^{(a)} & =\frac{1}{\sqrt{3}} \sum_{m} e^{-i m^{\prime} \phi_{m^{\prime}}} \alpha_{m}^{(\mathbf{a})} \\
\alpha_{m^{\prime}}^{(\mathbf{a})} & =\frac{1}{\sqrt{3}} \sum_{m} e^{i m \phi_{m^{\prime}}} \alpha_{m}^{(a)}
\end{aligned}
$$

similar to the canonical transformation (67). Here

$$
\begin{aligned}
& D_{m}^{(a)}=\exp \left(\alpha_{m}^{(a)} a_{m}^{+}-\text {H.c. }\right) \\
& D_{m}^{(\mathbf{a})}=\exp \left(\alpha_{m}^{(\mathbf{a})} \mathbf{a}_{m}^{+}-\text {H.c. }\right)
\end{aligned}
$$

are the "dual" Glauber displacement operators [81] (H.c. denotes Hermitian conjugation). If we consider, as an example, the state $\left|\alpha_{+} ; 0 ; 0\right\rangle$ of the electric dipole radiation with only one component $m=+1$, we will see that it is represented by the dual coherent state

$$
\bigotimes_{m}\left|\alpha_{m}^{(\mathbf{a})}\right\rangle, \quad \alpha_{m}^{(\mathbf{a})}=\frac{1}{\sqrt{3}} \alpha_{+}^{(a)} e^{i 2 m \pi / 3}
$$

in which all the three "phase" components of the electric dipole radiation are in the coherent states. 
The dual representation of the photon operators (67) reflects the transmission of "phase information" from the atomic transition to the radiation field via the integral of motion (70). This statement can be illustrated with the aid of the Jaynes-Cummings model (34). Employing the atomic phase states (46), we can introduce the dual representation of the atomic operators (35) as follows:

$$
R_{m m^{\prime}}^{(\phi)} \equiv\left|\phi_{m}\right\rangle\left\langle\phi_{m^{\prime}}\left|, \quad R_{m g}^{(\phi)} \equiv\right| \phi_{m}\right\rangle\langle g|
$$

Then, the simultaneous use of the dual representation of the atomic operators (75) and the canonical transformation of photon operators (67) leads to the following form of the Jaynes-Cummings Hamiltonian (34):

$$
\begin{aligned}
& H^{(\phi)}=H_{0}^{(\phi)}+H_{\mathrm{int}}^{(\phi)} \\
& H_{0}^{(\phi)}=\sum_{m=-1}^{1}\left[\omega \mathbf{a}_{m}^{+} \mathbf{a}_{m}+\omega_{0} R_{m m}^{(\phi)}\right] \\
& \left.H_{\mathrm{int}}^{(\phi)}=+g\left(R_{m g}^{(\phi)} \mathbf{a}_{m}+\mathbf{a}_{m}^{+} R_{g m}^{(\phi)}\right)\right]
\end{aligned}
$$

which has exactly the same operator structure as (34) in the dual representation [46]. Since the dual atomic operators (75) describe the transition between the atomic phase states and ground state, and the operators $\mathbf{a}_{m}$ and $\mathbf{a}_{m}^{+}$determine the annihilation and creation of photons with given radiation phase, the interaction term in (76) describes the transmission of the quantum phase information from the atom to photons.

We now note that the quantum phase in the Jaynes-Cummings model has been examined in a huge number of papers (for reviews, see Refs. 39 and 68). Most of them are based on the approach proposed in the pioneering paper by Dirac [1] and developed by a number of authors. Among the principal contributions to the field, the Pegg-Barnett approach [45] should be mentioned as the currently most popular. The main idea of the approach consists in defining the quantum phase operator first in a finite $s$-dimensional subspace of the infinite-dimensional Hilbert space $\mathscr{H}_{\text {field }}$ with subsequent formal limit transition $s \rightarrow \infty$, which is taken only after the averages have been calculated. In contrast, we consider the extended space of states $\mathscr{H}_{a} \otimes \mathscr{H}_{\text {field }}$ in which the quantum phase of radiation is defined by mapping of corresponding atomic operators from $\mathscr{H}_{a}$ into the whole Hilbert space $\mathscr{H}_{\text {field }}(60)$, using the conservation of angular momentum. In view of the dual form of the Jaynes-Cummings Hamiltonian (76), it is possible to say that the radiation phase is expressed in terms of what can be generated by a given quantum source.

So far our observations have been applied only to electric dipole radiation. It is straightforward to find the general form of the canonical transformation (67) 
in the same way as above. In the case of an arbitrary monochromatic pure $(\lambda, j)$ pole radiation, we get [46]

$$
\mathbf{a}_{\lambda k j m}=\frac{1}{\sqrt{2 j+1}} \sum_{m^{\prime}=-j}^{j} e^{i m^{\prime} \phi_{j m}} a_{\lambda k j m^{\prime}}, \quad \phi_{j m}=\frac{2 \pi m}{2 j+1}
$$

Here we again put $\psi=0$.

\section{Structure of Radiation Phase}

We now examine the spectrum of radiation phase constructed in the preceding subsection. Consider the state

$$
\left|\phi^{(\mathrm{rad})}\right\rangle=\bigotimes_{m=-1}^{1}\left|v_{m}\right\rangle
$$

where $\left|v_{m}\right\rangle$ is the radiation phase state (72). It is clear that (78) is the eigenstate of the operator (73). Since the operator (73) commute with the total number of photons

$$
N=\sum_{m=-1}^{1} a_{m}^{+} a_{m}=\sum_{m=-1}^{1} \mathbf{a}_{m}^{+} \mathbf{a}_{m}
$$

the eigenstates and eigenvalues of $\varepsilon_{\text {rad }}^{(\phi)}$ can be specified by the index

$$
n=\sum_{m=-1}^{1} v_{m}
$$

describing the total number of photons in a given state (72) and (74) and by an additional index $\ell$, describing a given distribution of $n$ photons over the three independent phase components of the electric dipole radiation in (72) and (74). The total number of possible different values of $\ell$, corresponding to a given $n$, is clearly

$$
\frac{1}{2}(n+2)(n+1)
$$

Then

$$
\varepsilon_{\mathrm{rad}}^{(\phi)}\left|\phi_{n \ell}^{(\mathrm{rad})}\right\rangle=e^{i \varphi_{n \ell}} \kappa_{n \ell}\left|\phi_{n \ell}^{(\mathrm{rad})}\right\rangle
$$


where $\left|\phi_{n \ell}^{(\mathrm{rad})}\right\rangle$ denotes the state (78) at given $n$ and $\ell$. The modulus of the eigenstates in (79) is determined as

$$
\begin{aligned}
\kappa_{n \ell}^{2}=\left\langle\phi_{n \ell}^{(\mathrm{rad})}\left|\varepsilon_{\mathrm{rad}}^{(\phi)}\left(\varepsilon_{\mathrm{rad}}^{(\phi)}\right)^{+}\right| \phi_{n \ell}^{(\mathrm{rad})}\right\rangle & =\sum_{m m^{\prime}} v_{m} v_{m}^{\prime} e^{i\left(m-m^{\prime}\right) 2 \pi / 3} \\
& =\sum_{m} v_{m}^{2}-\left(v_{+} v_{0}+v_{0} v_{-}+v_{-} v_{+}\right) \\
& =n^{2}+2\left(v_{+}^{2}+v_{-}^{2}\right)-3 n\left(v_{+}+v_{-}\right)+3 v_{+} v_{-}
\end{aligned}
$$

In turn, for the "phase eigenvalues" $\varphi_{n \ell}$ in (79), we get [46]

$$
\tan \phi=\frac{\sqrt{3}\left(v_{+}-v_{-}\right)}{2 v_{0}-\left(v_{+}+v_{-}\right)}
$$

Taking into account the physical meanings of the atomic operators (41) and (66) and the integrals of motion (64), we can consider the field operators (63) and (69) as the nonnormalized exponential operators of the radiation phase, which, by construction, is the $S U(2)$ phase of the multipole (electric dipole) radiation. By performing a similar analysis to that described in Section III.B, we can define the cosine and sine operators of the radiation phase as follows [36]

$$
C_{\mathrm{rad}}=K\left(\varepsilon_{\mathrm{rad}}+\varepsilon_{\mathrm{rad}}^{+}\right), \quad S_{\mathrm{rad}}=-i K\left(\varepsilon_{\mathrm{rad}}+\varepsilon_{\mathrm{rad}}^{+}\right)
$$

where $K$ is the normalization coefficient determined from the natural condition

$$
\left\langle C_{\mathrm{rad}}^{2}+S_{\mathrm{rad}}^{2}\right\rangle=1
$$

where $\langle\cdots\rangle$ is the averaging over the states of the electric dipole radiation under consideration. It is clear that $C_{\text {rad }}$ and $S_{\text {rad }}$ are commuting Hermitian operators so that corresponding physical quantities can be measured at once. In the dual representation provided by the canonical transformation (67), the operators (82) take the diagonal form

$$
\begin{aligned}
C_{\mathrm{rad}}^{(\phi)} & =K \sum_{m=-1}^{1} \mathbf{a}_{m}^{+} \mathbf{a}_{m} \cos \phi_{m} \\
S_{\mathrm{rad}}^{(\phi)} & =K \sum_{m=-1}^{1} \mathbf{a}_{m}^{+} \mathbf{a}_{m} \sin \phi_{m}
\end{aligned}
$$


Therefore, averaging over the phase states (78), we get

$$
\begin{aligned}
\left\langle\phi_{n \ell}^{(\mathrm{rad})}\left|C_{\mathrm{rad}}^{(\phi)}\right| \phi_{n \ell}^{(\mathrm{rad})}\right\rangle=2 \kappa_{n \ell} \cos \varphi_{n \ell} \\
\left\langle\phi_{n \ell}^{(\mathrm{rad})}\left|S_{\mathrm{rad}}^{(\phi)}\right| \phi_{n \ell}^{(\mathrm{rad})}\right\rangle=2 \kappa_{n \ell} \sin \varphi_{n \ell}
\end{aligned}
$$

According to the condition (83), we obtain

$$
K=\frac{1}{2 \kappa_{n \ell}}
$$

in this case. Hence, the radiation phase states (78) are the eigenstates of the operators (84), which, because of their structure, can be interpreted as the cosine and sine of the radiation phase operators. It is interesting that the eigenvalues of the radiation phase variable defined by $(81)$ belong to the interval $(0,2 \pi)$ and form a discrete set for any finite number of photons $n$ and

$$
\ell=1,2, \ldots, \frac{(n+1)(n+2)}{2}
$$

The first few eigenvalues are shown in Table I and Fig. 5.

It is not difficult to see that the vacuum averages of the operators (84) have the form

$$
\left\langle 0\left|C_{\mathrm{rad}}^{(\phi)}\right| 0\right\rangle=\left\langle 0\left|S_{\mathrm{rad}}^{(\phi)}\right| 0\right\rangle=0
$$

TABLE I

List of Eigenvalues $(81)^{a}$

\begin{tabular}{lll}
\hline$n=1$ & $\epsilon^{(1)}=1$ & $\varphi^{(1)}=2 \ell \pi / 3$ \\
$n=2$ & $\epsilon^{(2)}=2$ & $\varphi^{(2)}=2 \ell \pi / 3$ \\
& $\epsilon^{(2)}=1$ & $\varphi^{(2)}=(2 \ell+1) \pi / 3$ \\
$n=3$ & $\epsilon^{(3)}=3$ & $\varphi^{(3)}=2 \ell \pi / 3$ \\
$n=4$ & $\epsilon^{(3)}=\sqrt{3}$ & $\phi^{(3)}= \pm(\pi / 2+\ell \pi / 3)$ \\
& $\epsilon^{(4)}=4$ & $\varphi^{(4)}=2 \ell \pi / 3$ \\
& $\epsilon^{(4)}=\sqrt{7}$ & $\varphi^{(4)}= \pm \tan ^{-1}(\sqrt{3} / 5)+2 \ell \pi / 3$ \\
& $\epsilon^{(4)}=2$ & $\varphi^{(4)}=(2 \ell+1) \pi / 3$ \\
& $\epsilon^{(4)}=1$ & $\varphi^{(4)}=2 \ell \pi / 3$ \\
& $\epsilon^{(5)}=5$ & $\varphi^{(5)}=2 \ell \pi / 3$ \\
& $\epsilon^{(5)}=\sqrt{13}$ & $\varphi^{(5)}=\tan ^{-1}(\sqrt{3} / 7) \pm 2 \ell \pi / 3$ \\
& $\epsilon^{(5)}=\sqrt{7}$ & $\varphi^{(5)}= \pm \tan ^{-1}(\sqrt{3} / 7)+2 \ell \pi / 3$ \\
& $\epsilon^{(5)}=2$ & $\varphi^{(5)}= \pm \tan ^{-1}(\sqrt{3} / 5)+\pi+2 \ell \pi / 3$ \\
& $\epsilon^{(5)}=1$ & $\varphi^{(5)}=2 \ell \pi / 3$ \\
\hline
\end{tabular}

${ }^{a}$ Here $\ell=0, \pm$. 

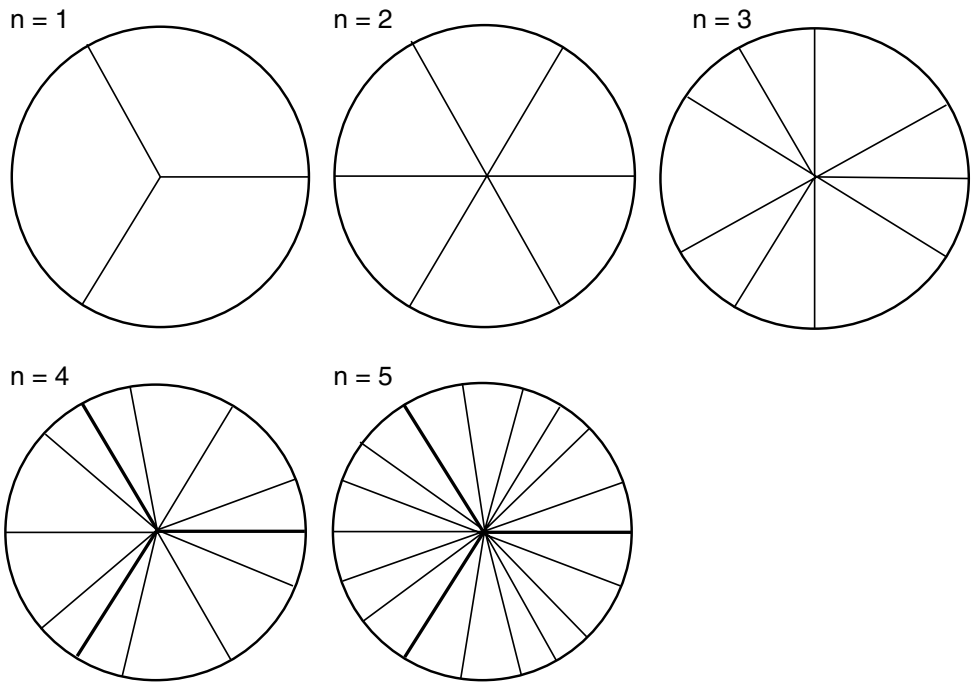

Figure 5. The structure of eigenvalues (80)-(81) corresponding to the phase states with $n=1, \ldots, 5$ photons. The bold lines correspond to the double-degenerated eigenstates.

while the vacuum variances

$$
\left\langle 0\left|\left(\Delta C_{\text {rad }}^{(\phi)}\right)^{2}\right| 0\right\rangle=\left\langle 0\left|\left(\Delta S_{\text {rad }}^{(\phi)}\right)^{2}\right| 0\right\rangle=\frac{1}{2}
$$

Hence, as all one can expect, the vacuum distribution of the radiation phase is uniform.

Now consider the electric dipole radiation with coherent components $m= \pm 1$, while the component $m=0$ is in the vacuum state:

$$
|\alpha\rangle=\left|\alpha_{+}\right\rangle \otimes|0\rangle \otimes\left|\alpha_{-}\right\rangle
$$

at $\left|\alpha_{+}\right|=\left|\alpha_{-}\right| \equiv|\alpha|$. In this case, the condition (83) gives

$$
K=\frac{1}{\sqrt{4|\alpha|^{2}\left(2+|\alpha|^{2}\right)}}
$$

so that

$$
\begin{aligned}
\left.\left\langle C_{\mathrm{rad}}^{(\phi)}\right)\right\rangle & =\frac{|\alpha|}{\sqrt{2+|\alpha|^{2}}} \cos \Delta_{+-} \\
\left.\left\langle S_{\mathrm{rad}}^{(\phi)}\right)\right\rangle & =\frac{|\alpha|}{\sqrt{2+|\alpha|^{2}}} \sin \Delta_{+-}
\end{aligned}
$$


where

$$
\Delta_{+-} \equiv \arg \alpha_{+}-\arg \alpha_{-}
$$

One can see that at $|\alpha| \rightarrow \infty$ we get

$$
\left.\left.\left\langle C_{\mathrm{rad}}^{(\phi)}\right)\right\rangle \rightarrow \cos \Delta_{+-}, \quad\left\langle S_{\mathrm{rad}}^{(\phi)}\right)\right\rangle \rightarrow \sin \Delta_{+-}
$$

so that in the classical limit of infinitely many coherent photons the operators (113) define the cosine and sine of the phase difference between the two components of the radiation field (85). In turn, for the variances we get

$$
\left\langle 0\left|\left(\Delta C_{\text {rad }}^{(\phi)}\right)^{2}\right| 0\right\rangle=\left\langle 0\left|\left(\Delta S_{\text {rad }}^{(\phi)}\right)^{2}\right| 0\right\rangle=\frac{2+\cos \Delta_{+-}}{2\left(2+|\alpha|^{2}\right)}
$$

Here the right-hand side tends to zero when $|\alpha| \rightarrow \infty$. Hence, the radiation phase has the natural classical limit.

At first sight, Eq. (87) gives wrong limit at $|\alpha| \rightarrow 0$ :

$$
\lim _{|\alpha| \rightarrow 0}\left\langle 0\left|\left(\Delta C_{\mathrm{rad}}^{(\phi)}\right)^{2}\right| 0\right\rangle=\lim _{|\alpha| \rightarrow 0}\left\langle 0\left|\left(\Delta S_{\mathrm{rad}}^{(\phi)}\right)^{2}\right| 0\right\rangle=\frac{2+\cos \Delta_{+-}}{4}
$$

In fact, this is an illusory contradiction. Because of the degeneration of the vacuum state with respect to the phase difference, the limit transition $|\alpha| \rightarrow 0$ should imply the averaging over all possible $\Delta \in(0,2 \pi)$, which leads to the natural value of the vacuum variances.

Now consider the case when $\left|\alpha_{+}\right| \neq\left|\alpha_{-}\right|$in the state (85). Then, instead of (86) and (87), we get

$$
\begin{aligned}
\left.\left\langle C_{\mathrm{rad}}^{(\phi)}\right)\right\rangle & =\frac{\cos \Delta_{+-}}{\sqrt{1+\left|\alpha_{+}\right|^{-2}+\left|\alpha_{-}\right|^{-2}}} \\
\left.\left\langle S_{\mathrm{rad}}^{(\phi)}\right)\right\rangle & =\frac{\sin \Delta_{+-}}{\sqrt{1+\left|\alpha_{+}\right|^{-2}+\left|\alpha_{-}\right|^{-2}}}
\end{aligned}
$$

and

$$
\begin{aligned}
\left\langle\left(\Delta C_{\mathrm{rad}}^{(\phi)}\right)^{2}\right\rangle & =\left\langle\left(\Delta S_{\mathrm{rad}}^{(\phi)}\right)^{2}\right\rangle \\
& =\frac{\left|\alpha_{+}\right|^{2}+\left|\alpha_{-}\right|^{2}+\left|\alpha_{+}\right|\left|\alpha_{-}\right| \cos \Delta_{+-}}{2\left(\left|\alpha_{+}\right|^{2}+\left|\alpha_{-}\right|^{2}+\left|\alpha_{+}\right|^{2}\left|\alpha_{-}\right|^{2}\right)}
\end{aligned}
$$


respectively. Examine now the averages (89) and variance (90) as a function of $\left|\alpha_{+}\right|$at fixed $\left|\alpha_{-}\right|[54]$. We obtain

$$
\left\{\begin{array}{l}
\left.\left\langle C_{\mathrm{rad}}^{(\phi)}\right)\right\rangle \rightarrow 0 \\
\left.\left\langle S_{\mathrm{rad}}^{(\phi)}\right)\right\rangle \rightarrow 0 \\
\left\langle 0\left|\left(\Delta C_{\mathrm{rad}}^{(\phi)}\right)^{2}\right| 0\right\rangle \rightarrow \frac{1}{2} \\
\left\langle 0\left|\left(\Delta S_{\mathrm{rad}}^{(\phi)}\right)^{2}\right| 0\right\rangle \rightarrow \frac{1}{2}
\end{array}\right.
$$

because the operators (84) define properties of the relative phase (phase difference between the components) which does not exist when only component $m=-1$ is emitted. It is seen that, under the condition

$$
\left|\alpha_{+}\right|\left|\alpha_{-}\right|<\cos \Delta_{+-} \leq 1
$$

which can be realized in the strong quantum case of very low intensities, the value of the variances (90) can exceed the vacuum limit of $\frac{1}{2}$. The maximum in (90) is achieved at

$$
\left|\alpha_{+}\right|=\left|\alpha_{-}\right| \times \frac{\sqrt{\left|\alpha_{-}\right|^{4}+\left(1+\left|\alpha_{-}\right|^{2}\right) \cos \Delta_{+-}}-\left|\alpha_{-}\right|^{2}}{\left(1+\left|\alpha_{-}\right|^{2}\right) \cos \Delta_{+-}}
$$

The dependence of the variances (90) on $\left|\alpha_{+}\right|$at fixed $\left|\alpha_{-}\right|$is shown in Fig. 6 . The qualitative explanation of this effect of strong increase of quantum fluctuations in the low-intensity limit is based on the consideration of the probability to have a given phase difference $\Delta_{+-}$. At $\left|\alpha_{+}\right|=0$, there is a uniform probability distribution in the system, causing the limit relations (91). Creation of very few photons of the mode $m=+1$ leads to the formation of some domains with almost equal probabilities having phase differences $\Delta_{+-}$ and $\Delta_{ \pm}+\pi$. So, it looks like a "phase bunching" (for the bunching of photons, see Refs. 14 and 15). Further increase of $\left|\alpha_{+}\right|$leads to formation of a more or less sharp probability distribution that cannot reach the $\delta$-function shape because the variances (90) achieve the saturation described by the expression

$$
\left.\left.\lim _{\left|\alpha_{+}\right| \rightarrow \infty}\left\langle C_{\mathrm{rad}}^{(\phi)}\right)\right\rangle=\lim _{\left|\alpha_{+}\right| \rightarrow \infty}\left\langle S_{\mathrm{rad}}^{(\phi)}\right)\right\rangle=\frac{1}{2\left(1+\left|\alpha_{-}\right|^{2}\right)}
$$

which coincides with the classical limit only if $\left|\alpha_{-}\right| \rightarrow \infty$ as well. This means that the presence of one quantum component in the coherent state (85) leads to quantum phase fluctuations. 


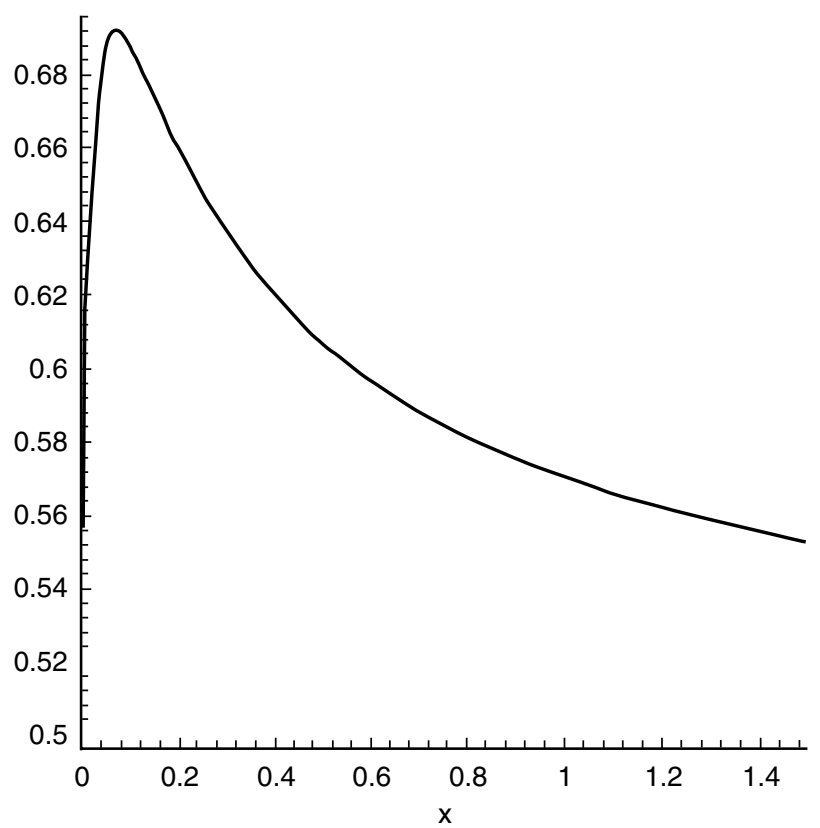

Figure 6. The dependence of variances (90) on $\left|\alpha_{+}\right|$at fixed $\left|\alpha_{-}\right|=0.275$ and $\Delta_{+_{-}}=\pi / 6$.

Using the standard representation of Glauber coherent states in terms of the number states of photons [14,81]

$$
|\alpha\rangle=e^{-|\alpha|^{2} / 2} \sum_{n=0}^{\infty} \frac{\alpha^{n}}{\sqrt{n !}}|n\rangle
$$

and making use of the relation (74), we can represent the coherent state (85) in the following way

$$
\left|\alpha_{+} ; 0 ; \alpha_{-}\right\rangle=\prod_{m=-1}^{1} e^{-i\left|\alpha_{m}^{(\mathbf{a})}\right|^{2} / 2} \sum_{v_{m}=0}^{\infty} \frac{\left(\alpha_{m}^{(\mathbf{a})}\right)^{v_{m}}}{\sqrt{v_{m}}}\left|v_{m}\right\rangle
$$

where the parameters $\alpha_{m}^{(\mathbf{a})}$ have been defined at the end of Section IV.A. Assume that $\left|\alpha_{+}\right|=\left|\alpha_{-}\right| \equiv|\alpha|$. Then

$$
\sum_{m}\left|\alpha_{m}^{(\mathbf{a})}\right|^{2}=2|\alpha|^{2}
$$


and the probability of observing the radiation field in a given phase state (72), (74) is

$\left|\left\langle v_{+} ; v_{0} ; v_{-} \mid \alpha_{+} ; 0 ; \alpha_{-}\right\rangle\right|^{2}=e^{-2|\alpha|^{2}}\left(\frac{2}{3}\right)^{n} \frac{|\alpha|^{2 n}}{v_{+} ! v_{0} ! v_{-} !} \prod_{m=-1}^{1}\left[1+\cos \left(\Delta_{+-}-\frac{2 m \pi}{3}\right)\right]^{v_{m}}$

where $n=\sum_{m} v_{m}$. It is easily seen that this probability tends to zero when $|\alpha| \rightarrow 0$ or $|\alpha| \rightarrow \infty$. This means that the eigenvalues of the radiation phase are distributed uniformly over the interval $(0,2 \pi)$ in the vacuum state as well as in the classical limit of high-intensity coherent state. Between these two extrema, the probability (93) has a maximum, which can be considerably high. It is interesting that the position of the maximum is completely determined by the mean number of photons $\left|\alpha_{\max }\right|^{2}=n$, while the magnitude also depends on the phase difference $\Delta_{+-}$(see Fig. 7).

Let us now calculate the probability to have a given value of the radiation phase in the coherent state under consideration. Consider, for example, the eigenvalue of the radiation phase $\varphi=2 \pi / 3$. Employing equations (80) and (81) then gives the following properties of the states corresponding to this radiation phase:

$$
n-3 v_{-}=\kappa_{n} \quad v_{+}=n-2 v_{-} \quad v_{0}=v_{-}
$$

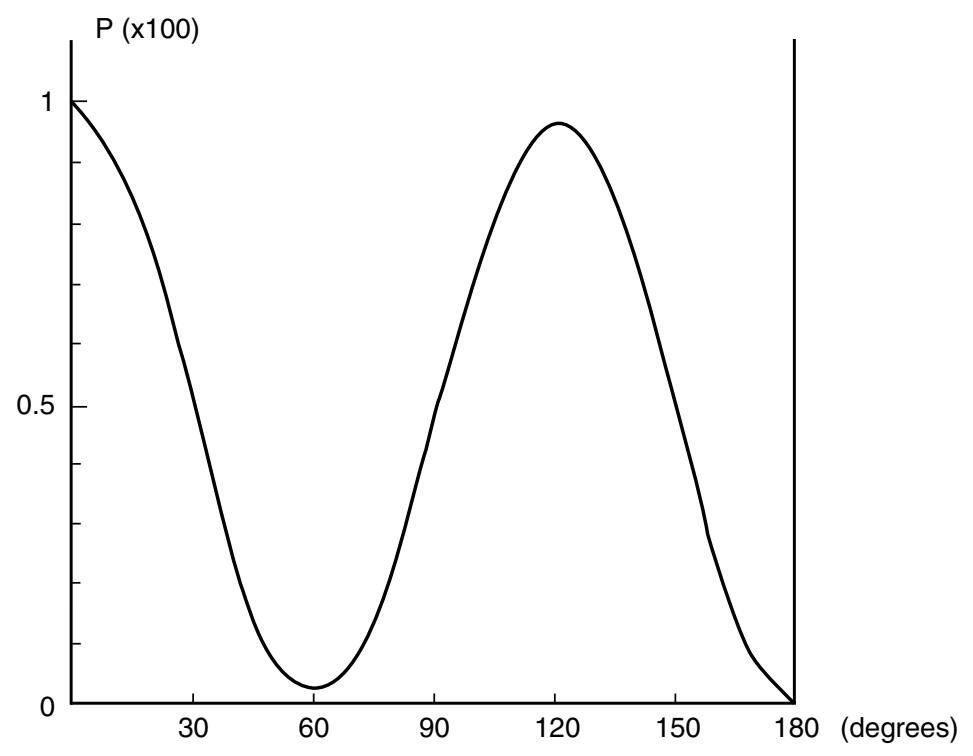

Figure 7. Probability (93) versus $\Delta_{+-}$. 
It is easy to see that the states obeying these conditions have the following structure:

$$
\left|n_{p}-2 k_{p} ; k_{p} ; k_{p}\right\rangle
$$

where $p$ is an integer and for each $n_{p}=3 p, 3 p-1,3 p-2$ the numbers $k_{p}$ take the values $k_{p}=0,1, \ldots, p-1$. For example, at $n_{p}=10$, we get $p=4$ and $n_{p}=3 p-2$, while the states (94) are

$$
|10 ; 0 ; 0\rangle, \quad|8 ; 1 ; 1\rangle, \quad|6 ; 2 ; 2 ;\rangle, \quad|4 ; 3 ; 3\rangle
$$

Consider first the states $\left|n_{p} ; 0 ; 0\right\rangle$ in (94). Then, the probability (93) takes the value

$$
\begin{aligned}
\mathscr{P}_{n_{p}} \equiv & \left|\left\langle n_{p} ; 0 ; 0 \mid \alpha_{+} ; 0 ; \alpha_{-}\right\rangle\right|^{2} \\
& \times e^{-2|\alpha|^{2}}\left(\frac{2}{3}\right)^{n_{p}} \frac{|\alpha|^{2 n_{p}}}{n_{p} !}\left[1+\cos \left(\Delta_{+-}-\frac{2 \pi}{3}\right)\right]^{n_{p}}
\end{aligned}
$$

It is then clear that $\mathscr{P}_{n_{p}}$ reaches its maximum at $\Delta_{+-}=2 \pi / 3$. Then, the total probability to have the phase states $\left|n_{p} ; 0 ; 0\right\rangle$ is

$$
\mathscr{P}=\sum_{n_{p}=1}^{\infty} \mathscr{P}_{n_{p}}=e^{-2|\alpha|^{2} / 3}-e^{-2|\alpha|^{2}} \geq 0
$$

(see Fig. 8). It is clear that this function $\mathscr{P}$ gives the lower bond of the total probability to observe the radiation phase $\varphi=2 \pi / 3$ in the coherent state under consideration. The contribution of the other states (94) can be calculated in the same way.

\section{Radiation Phase in Jaynes-Cummings Model}

To illustrate the exchange of the phase information between the atomic transition and the multipole field, consider the electric dipole Jaynes-Cummings model (34). Assume that the field consists of two circularly polarized components in a coherent state each. The atom is supposed to be initially in the ground state. Then, the time-dependent wave function of the system has the form [53]

$$
\begin{aligned}
|\Psi(t)\rangle= & \sum_{n_{+}, n_{-}} P\left(n_{+}, n_{-}\right)[\cos (g \sqrt{n t})|0,0\rangle \\
& \left.+\left(\alpha_{+}|1,1\rangle+\alpha_{-}|1,-1\rangle\right) \xi\left(n_{+}, n_{-}\right)\right]\left|n_{+} n_{-}\right\rangle
\end{aligned}
$$




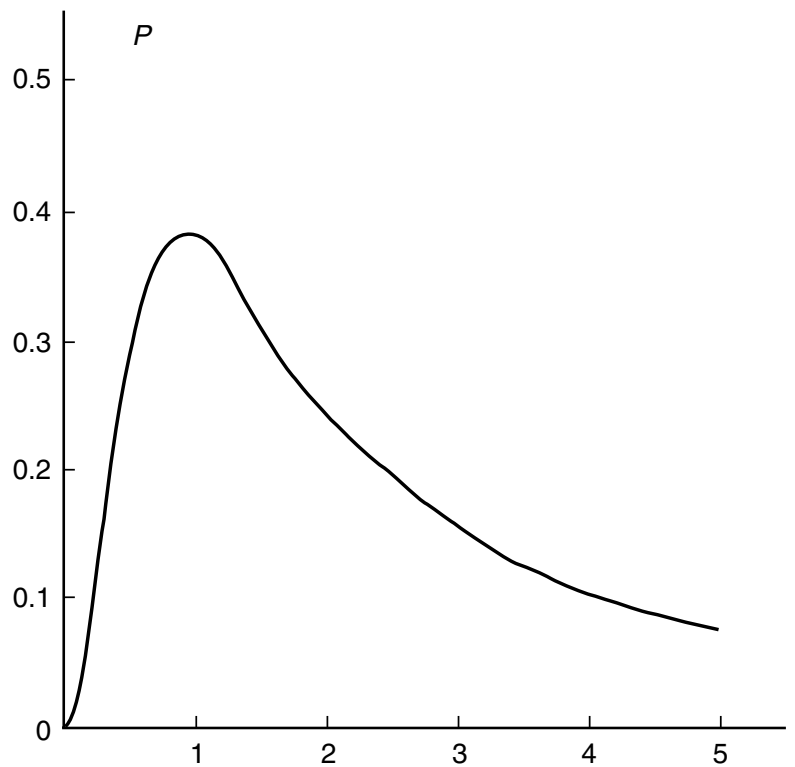

Figure 8. Lower estimate of the probability to have the radiation phase $\varphi=2 \pi / 3$ as a function of $|\alpha|^{2}$ at $\Delta_{+-}=2 \pi / 3$.

where $\left|n_{m}\right\rangle$ denotes the Fock number state with $n_{m}$ photons with the quantum number $m ;|1, m\rangle$ and $|0,0\rangle$ are the excited and ground atomic states, respectively; $g$ is the coupling constant in (34), $n=n_{+} n_{-}$

$$
P\left(n_{+}, n_{-}\right)=e^{-\left(\left|\alpha_{+}\right|^{2}-\left|\alpha_{-}\right|^{2}\right) / 2} \frac{\alpha_{+}^{n_{+}} \alpha_{-}^{n_{-}}}{\sqrt{n_{+} ! n_{-} !}}
$$

and

$$
\xi\left(n_{+}, n_{-}\right)=\frac{\sin (g t \sqrt{n+1})}{\sqrt{n+1}} e^{-i(n+1) \omega t}
$$

Then, the mean values of the operators (82), describing the cosine and sine of the radiation phase, take the form

$$
\begin{aligned}
\left\langle C_{\mathrm{rad}}\right\rangle_{t} & =\sqrt{\frac{\left|\alpha_{+}\right|^{2}\left|\alpha_{-}\right|^{2}}{\left|\alpha_{+}\right|^{2}\left|\alpha_{-}\right|^{2}+\left|\alpha_{+}\right|^{2}\left|\alpha_{-}\right|^{2}}} \frac{\left\langle a_{+}^{+} a_{+}+a_{-}^{+} a_{-}\right\rangle_{t}}{\left|\alpha_{+}\right|^{2}+\left|\alpha_{-}\right|^{2}} \cos \Delta_{+-} \\
\left\langle S_{\mathrm{rad}}\right\rangle_{t} & =\sqrt{\frac{\left|\alpha_{+}\right|^{2}\left|\alpha_{-}\right|^{2}}{\left|\alpha_{+}\right|^{2}\left|\alpha_{-}\right|^{2}+\left|\alpha_{+}\right|^{2}\left|\alpha_{-}\right|^{2}}} \frac{\left\langle a_{+}^{+} a_{+}+a_{-}^{+} a_{-}\right\rangle_{t}}{\left|\alpha_{+}\right|^{2}\left|+\alpha_{-}\right|^{2}} \sin \Delta_{+-}
\end{aligned}
$$


Here

$$
\left\langle a_{+}^{+} a_{+}+a_{-}^{+} a_{-}\right\rangle_{t}=\left|\alpha_{+}\right|^{2}+\left|\alpha_{-}\right|^{2}-\sum_{n_{+}, n_{-}=0}^{\infty}\left|P\left(n_{+}, n_{-}\right)\right|^{2} \sin ^{2} g t \sqrt{n_{+}+n_{-}}
$$

and $\Delta_{+-} \equiv \arg \alpha_{+}-\arg \alpha_{-}$. Thus, the averages (97) describe the Rabi oscillations of cosine and sine of the phase difference between the two coherent components of the field. Corresponding variances have the form

$$
\begin{aligned}
\left\langle\left(\Delta C_{\mathrm{rad}}\right)^{2}\right\rangle_{t} & =\frac{\left\langle a_{+}^{+} a_{+}+a_{-}^{+} a_{-}\right\rangle_{t}}{\left|\alpha_{+}\right|^{2}+\left|\alpha_{-}\right|^{2}}\left[\left\langle\left(\Delta C_{\mathrm{rad}}\right)^{2}\right\rangle_{0}+\beta_{C} Q\right] \\
\left\langle\left(\Delta S_{\mathrm{rad}}\right)^{2}\right\rangle_{t} & =\frac{\left\langle a_{+}^{+} a_{+}+a_{-}^{+} a_{-}\right\rangle_{t}}{\left|\alpha_{+}\right|^{2}+\left|\alpha_{-}\right|^{2}}\left[\left\langle\left(\Delta S_{\mathrm{rad}}\right)^{2}\right\rangle_{0}+\beta_{S} Q\right]
\end{aligned}
$$

Here

$$
\begin{aligned}
\left\langle\left(\Delta C_{\mathrm{rad}}\right)^{2}\right\rangle_{0} & =\frac{1}{2} \cos \Delta_{+-} \\
\left\langle\left(\Delta S_{\mathrm{rad}}\right)^{2}\right\rangle_{0} & =\frac{|\alpha|^{2}+\left|\alpha_{-}\right|^{2}-\left|\alpha_{+}\right|\left|\alpha_{-}\right|}{|\alpha|^{2}+\left|\alpha_{-}\right|^{2}+\left|\alpha_{+}\right|\left|\alpha_{-}\right|} \cos \Delta_{+-}
\end{aligned}
$$

and

$$
\begin{aligned}
& \beta_{C}=\frac{\left|\alpha_{+} \| \alpha_{-}\right|}{\left(|\alpha|^{2}+\left|\alpha_{-}\right|^{2}+\left|\alpha_{+}\right|\left|\alpha_{-}\right|\right)\left(|\alpha|^{2}+\left|\alpha_{-}\right|^{2}+\left|\alpha_{+} \| \alpha_{-}\right|\right)} \cos ^{2} \Delta_{+-} \\
& \beta_{C}=\frac{\left|\alpha_{+} \| \alpha_{-}\right|}{\left(|\alpha|^{2}+\left|\alpha_{-}\right|^{2}+\left|\alpha_{+} \| \alpha_{-}\right|\right)\left(|\alpha|^{2}+\left|\alpha_{-}\right|^{2}+\left|\alpha_{+} \| \alpha_{-}\right|\right)} \sin ^{2} \Delta_{+-}
\end{aligned}
$$

In (98), $Q$ is the Mandel factor [14], describing the deviation of the photon statistics from the Poisson distribution for the total intensity:

$$
Q=\frac{\left\langle\left[\Delta\left(a_{+}^{+} a_{+}+a_{-}^{+} a_{-}\right)\right]^{2}\right\rangle-\left\langle\left(a_{+}^{+} a_{+}+a_{-}^{+} a_{-}\right)\right\rangle}{\left\langle\left(a_{+}^{+} a_{+}+a_{-}^{+} a_{-}\right)\right\rangle}
$$

It is seen that the time-averaged Mandel $Q$ factor is always positive, which shows the super-Poissonian number distribution for the total field.

Since $\left\langle C_{\mathrm{rad}}\right\rangle_{t}$ and $\left\langle\left(\Delta C_{\mathrm{rad}}\right)^{2}\right\rangle_{t}$ can be transformed into $\left\langle S_{\mathrm{rad}}\right\rangle_{t}$ and $\left\langle\left(\Delta S_{\mathrm{rad}}\right)^{2}\right\rangle_{t}$, respectively, by the change of phase difference

$$
\Delta_{+-} \rightarrow \Delta_{+-}+\frac{k \pi}{2}
$$




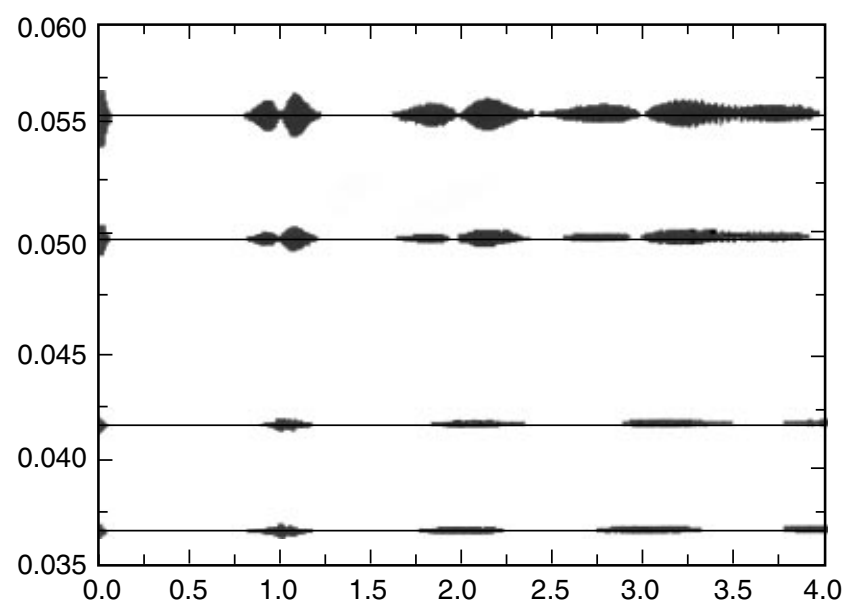

Figure 9. Rabi oscillations of the variance of the field cosine operator as a function of scaled time $t_{s}=g t /\left(2 \pi \sqrt{\left.n_{+}+n_{-}\right)}\right.$at $n_{ \pm}=25$ and $g=1$. Graphs from top to bottom correspond to the relative phase $\Delta_{+-}=0^{\circ}, 45^{\circ}, 75^{\circ}, 90^{\circ}$, respectively.

it suffices to examine only one pair of these functions. In Fig. 9, the Rabi oscillations of the variance $\left\langle\left(\Delta C_{\mathrm{rad}}\right)^{2}\right\rangle_{t}$ are shown as a function of time for different $\Delta_{+-}$. At small $\Delta_{+-}$, the collapse and revival picture of Rabi oscillations behaves quite typically for the Jaynes-Cummings model [68], while the increase of $\Delta_{+-}$leads to a confluence of the nearest revivals. The Rabi oscillations of $\left\langle C_{\mathrm{rad}}\right\rangle_{t}$ have similar behavior.

It should be emphasized that the system under consideration completely describes the process of transmission of the phase information between the field and the atom. Initially the atom is in the ground state with the angular momentum 0 and has no $S U(2)$ atomic phase at all. Absorption of photons induces an atomic phase that coincides with the phase difference between the two coherent components of the field. This can be concluded from a direct calculation of the expectation value of the atomic cosine operator (43) over the state (96):

$$
\left\langle C_{a}\right\rangle_{t}=\sum_{n_{+}, n_{-}=0}^{\infty}\left|P\left(n_{+}, n_{-}\right)\right|^{2} \frac{\sin ^{2}(g t \sqrt{n+1})}{n+1} \cos \Delta_{+-}
$$

This again clearly demonstrates the one-to-one correspondence between the atomic $S U(2)$ phase and the radiation phase.

Let us also stress that, according to (98), the variances of the cosine and sine of the radiation phase can be measured in the same way as the Mandel $Q$ factor. 


\section{E. Radiation Phase and Pegg-Barnett Quantum Phase}

Following [54], let us compare the quantum statistical behavior of the radiation phase, constructed in Sections IV.A-IV.D and that obtained within the PeggBarnett approach [45], which has received a lot of attention since the early 1990s years and has led to many important results (for a review, see Refs. 39 and 40). We use here the form of the Pegg-Barnett approach considered in Ref. 82. The point is that Ref. 82 deals with a generalization of the PeggBarnett approach to the case of two circularly polarized modes. Then, the phase distribution over the phases of two circularly polarized modes is determined as

$$
P_{\psi}=\left|\left\langle\phi_{+}, \phi_{-} \mid \psi\right\rangle\right|^{2}
$$

where $\left|\phi_{+}, \phi_{-}\right\rangle$is the Susskind-Glogower phase state [41] and $|\psi\rangle$ is the state of the radiation field. To establish the connection with the results already obtained in this section, suppose that

$$
|\psi\rangle=\left|\alpha_{+}\right\rangle \otimes\left|0_{0}\right\rangle \otimes\left|\alpha_{-}\right\rangle
$$

Since the formalism of the radiation phase is focused on the phase difference between the components, we need to use the distribution function for the relative phase

$$
\phi=\phi_{+}-\phi_{-}
$$

We use here the notations of Refs. 54 and 82 . Referring to the procedure suggested in Refs. 82 and 83 to cast the range of $\phi$ into $2 \pi$ range from $4 \pi$ range, we take

$$
P_{2 \pi}=\sum_{n=0}^{\infty}\left|\left\langle\phi^{(n)} \mid \psi\right\rangle\right|^{2}
$$

where

$$
\left|\phi^{(n)}\right\rangle=\frac{1}{\sqrt{2 \pi}} \sum_{n_{+}=0}^{n} e^{i \phi n_{+}}\left|n_{+}\right\rangle \otimes\left|0_{0}\right\rangle \otimes\left|n-n_{+}\right\rangle
$$

Using this distribution function, one can calculate the mean value of any function $F(\phi)$ of the relative phase as follows [82]:

$$
\langle F(\phi)\rangle=\int_{-\pi}^{\pi} d \phi P_{2 \pi}(\phi) F(\phi)
$$


In the case under consideration, the relative phase represents the phase difference between the two circularly polarized components in coherent state. Then, for the Pegg-Barnett cosine of the relative phase, we get

$$
\left\langle\cos \phi_{\mathrm{PB}}\right\rangle=e^{\left(\left|\alpha_{+}\right|^{2}+\left|\alpha_{-}\right|^{2}\right)} \sum_{n_{ \pm}=0}^{\infty} \frac{\left|\alpha_{+}\right|^{2 n_{+}}\left|\alpha_{-}\right|^{2 n_{-}}}{n_{+} ! n_{-} !} \times \frac{\operatorname{Re}\left(\alpha_{+} \alpha_{-}^{*}\right)}{\sqrt{\left(n_{+}+1\right)\left(n_{-}+1\right)}}
$$

In turn, the average of the squared cosine takes the form

$$
\begin{aligned}
\left\langle\cos ^{2} \phi_{\mathrm{PB}}\right\rangle= & \frac{1}{2}+\frac{e^{\left(\left|\alpha_{+}\right|^{2}+\left|\alpha_{-}\right|^{2}\right)}}{2} \sum_{n_{ \pm}=0}^{\infty} \frac{\left|\alpha_{+}\right|^{2 n_{+}}\left|\alpha_{-}\right|^{2 n_{-}}}{n_{+} ! n_{-} !} \\
& \times \frac{\operatorname{Re}\left(\alpha_{+} \alpha_{-}^{*}\right)}{\sqrt{\left(n_{+}+2\right)\left(n_{+}+1\right)\left(n_{-}+2\right)\left(n_{-}+1\right)}}
\end{aligned}
$$

Here $\phi_{\mathrm{PB}}$ denotes the Pegg-Barnett quantum phase operator [45]. These expressions can be now compared with the results (89) and (90) for the radiation cosine and its variance. To clarify the difference between the two approaches, we represent (89) and (90) in the same manner as (100) and (101) [54]:

$$
\begin{aligned}
\left\langle C_{\mathrm{rad}}^{(\phi)}\right\rangle= & e^{\left(\left|\alpha_{+}\right|^{2}+\left|\alpha_{-}\right|^{2}\right)} \sum_{n_{ \pm}=0}^{\infty} \frac{\left|\alpha_{+}\right|^{2 n_{+}}\left|\alpha_{-}\right|^{2 n_{-}}}{n_{+} ! n_{-} !} \\
& \times \frac{\operatorname{Re}\left(\alpha_{+} \alpha_{-}^{*}\right)}{\sqrt{\left|\alpha_{+}\right|^{2}+\left|\alpha_{-}\right|^{2}+\left|\alpha_{+}\right|^{2}\left|\alpha_{-}\right|^{2}}}
\end{aligned}
$$

and

$$
\begin{aligned}
\left\langle\left(C_{\mathrm{rad}}^{(\phi)}\right)^{2}\right\rangle= & \frac{1}{2}+\frac{\left\langle C_{\mathrm{rad}}^{(\phi)}\right\rangle}{2 \sqrt{\left|\alpha_{+}\right|^{2}+\left|\alpha_{-}\right|^{2}+\left|\alpha_{+}\right|^{2}\left|\alpha_{-}\right|^{2}}} \\
& +\frac{\left[\operatorname{Re}\left(\alpha_{+} \alpha_{-}^{*}\right)\right]^{2}}{2\left(\left|\alpha_{+}\right|^{2}+\left|\alpha_{-}\right|^{2}+\left|\alpha_{+}\right|^{2}\left|\alpha_{-}\right|^{2}\right)} \sum_{n_{ \pm}+0}^{\infty} \frac{\left|\alpha_{+}\right|^{2 n_{+}}\left|\alpha_{-}\right|^{2 n_{-}}}{n_{+} ! n_{-} !}
\end{aligned}
$$

One can easily see that each term in the sums in Eqs. (100) and (101) has different normalization, while in Eqs. (102) and (103) all the terms have the same normalization factor related to our choice of the constant $K$ in (82) and (83). In addition, Eq. (103) contains an extra term proportional to $\left\langle C_{\mathrm{rad}}^{(\phi)}\right\rangle$. This term comes from the vacuum fluctuations related to the mode $m=0$. This causes a striking difference when one of the modes $m= \pm 1$ is in the quantum 


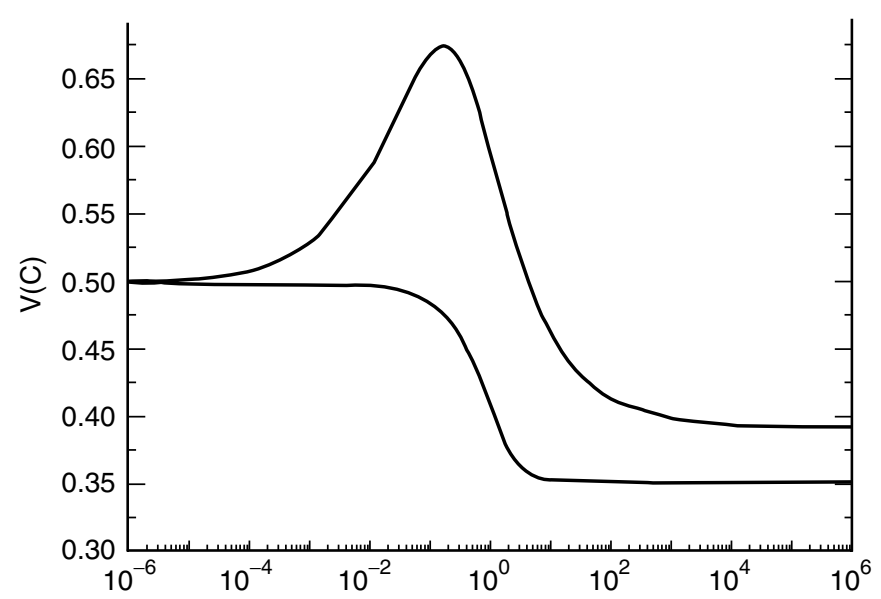

Figure 10. Variance of cosine. The lower curve represents the Pegg-Barnett cosine; the upper curve, the radiation phase cosine. Both curves are drawn at $\Delta_{+-}=0$ and $I_{-}=0.275$.

domain. Specifically, the existence of the "phase bunching" discussed in Section IV.C is caused just by this term. At the same time, both approaches show the saturation of the variances when one of the intensities tends to infinity while the second is kept constant (Fig. 10). It is seen that the quantum fluctuations of the phase difference between the two circularly polarized coherent fields with different helicity calculated with the aid of the radiation phase always exceed those calculated within the Pegg-Barnett approach.

This fact can be explained in the following way. The cutoff of the Hilbert space, which is a distinctive feature of the Pegg-Barnett approach [45], leads to an effective change of the algebraic properties of the photon operators. In fact, such a cutoff of the Fock basis leads to the definition of the unit operator, which can be considered as some approximation of the Casimir operator of the $S U(2)$ subalgebra, describing the angular momentum of radiation, but only in a particular subspace of the Hilbert space of photons. Existence of the unit operator makes it possible to perform direct polar decomposition and determine the corresponding quantum phase properties. This can be clearly traced in Ref. 84. At the same time, the cutoff procedure reduces the algebraic properties of photons responsible for the quantum fluctuations. The limit taken in the Pegg-Barnett approach after the calculation of all expectation values cannot completely restore these properties, which are especially important in the quantum domain.

Unlike the Pegg-Barnett approach, the definition of the radiation phase is based on the conservation laws for electromagnetic radiation and canonical 
transformation (67), which does not disturb the Weyl-Heisenberg algebra of the photon operators.

\section{F. Radiation Phase and Mandel's Operational Approach}

We now note that the operators (69) and (84) introducing the radiation phase are defined in terms of bilinear forms in the photon operators. At first glance, such a definition runs counter to the original idea by Dirac to determine the Hermitian quantum phase via linear forms in the photon operators [1] (see also Refs. 38, 42, and 44). Leaving aside Dirac's problem of existence of a Hermitian quantum phase variable of a harmonic oscillator, we should emphasize that the use of bilinear forms seems to be quite reasonable from the physical point of view. It can be argued in the following way:

1. The phase information is transmitted from the quantum source (atom) to photons via the conservation laws. In fact, only three physical quantities are conserved in the process of radiation: energy, linear momentum, and angular momentum [26]. All of them are represented by the bilinear forms in the photon operators.

2. The detection process is also based on the transmission of energy, linear momentum, and/or angular momentum from the photons to a detecting device [14]. In other words, the Hermitian bilinear forms in (84) corresponds to what can be emitted by the source and detected by a photodetector.

Let us stress that the operational definition of the quantum phase of radiation [47] is also based on the use of bilinear forms in the photon operators. In the simplest form, the idea of the operational approach to the phase difference can be illustrated with the aid of the two-port interferometer shown in Fig. 11 (see Refs. 14 and 47 for more detailed discussion). The two incident monochromatic (or quasimonochromatic) light beams are combined by a symmetric beamsplitter oriented at $45^{\circ}$ to each beam. The resultant intensities emerging from each output port are measured by the two photodetectors connected with a "comparator" (computer) as in the Hanbury-Brown-Twiss interferometer [85] (also see Refs. 14, 15, and 86). Following Noh et al. [47], we denote by $a_{1}$ and $a_{2}$ the photon annihilation operators, describing the field at the two input ports, and by $a_{3}$ and $a_{4}$ the corresponding operators at the two output ports. Then

$$
\begin{aligned}
& a_{3}=t a_{1}+r^{\prime} a_{2} \\
& a_{4}=r a_{1}+t^{\prime} a_{2}
\end{aligned}
$$

where $t$ and $r$ denote the complex-amplitude transmittance and reflectance from one side of the beamsplitter and $r^{\prime}$ and $t^{\prime}$ from the other side. The number of 


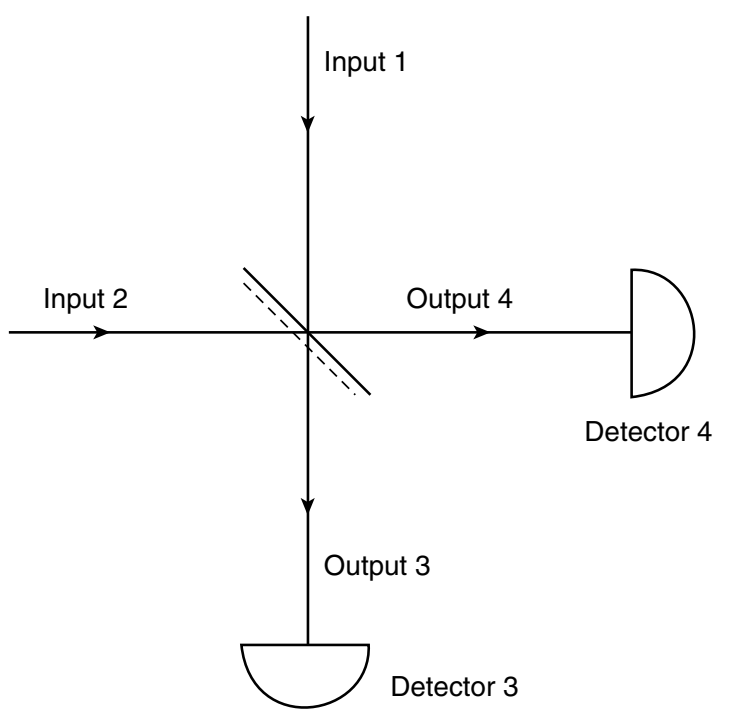

Figure 11. Outline of the scheme of two-part interferometer.

photons in the output modes is defined as follows

$$
\begin{aligned}
& \hat{n}_{3}=|t|^{2} \hat{n}_{1}+|r|^{2} \hat{n}_{2}+t^{*} r^{\prime} a_{1}^{+} a_{2}+t r^{\prime *} a_{2}^{+} a_{1} \\
& \hat{n}_{4}=|r|^{2} \hat{n}_{1}+|t|^{2} \hat{n}_{2}+t^{\prime} r^{*} a_{1}^{+} a_{2}+r t^{\prime *} a_{2}^{+} a_{1}
\end{aligned}
$$

where $\hat{n}_{\ell} \equiv a_{\ell}^{+} a_{\ell}$. It is clear that

$$
\left.\hat{n}_{4}-\hat{n}_{3}=\exp \left[i \arg r-\arg r^{\prime}\right)\right] a_{2}^{+} a_{1}+\exp \left[i\left(\arg t^{\prime}-\arg t\right)\right] a_{1}^{+} a_{2}
$$

Hence, the sine and cosine of the phase difference between the two output beams can be defined as follows [47]

$$
\begin{aligned}
S_{M} & =K_{1}\left(\exp \left[i\left(\arg r-\arg t^{\prime}\right)\right] a_{2}^{+} a_{1}+\exp \left[i\left(\arg t^{\prime}-\arg r\right)\right] a_{1}^{+} a_{2}\right) \\
C_{M} & =i K_{2}\left(\exp \left[i\left(\arg r-\arg t^{\prime}\right)\right] a_{2}^{+} a_{1}-\exp \left[i\left(\arg t^{\prime}-\arg r\right)\right] a_{1}^{+} a_{2}\right)
\end{aligned}
$$

where $K_{1}$ and $K_{2}$ are some constants. It is clear that, apart from the exponential factors caused by the measuring device, these equations for $S_{M}$ and $C_{M}$ have the operator structure similar to the cosine and sine operators of the radiation phase (82). Of course, the operational definition by Mandel et al. [47] discussed above does not take into consideration the third component of the multipole radiation. Therefore, the operators (104) do not commute with each other. 
From the preceding discussion, one can conclude that the method of radiation phase, defining the quantum phase variable in terms of what can be emitted by the source, complements the operational phase, which deals with what can be measured in a real experiment.

\section{G. Phase Properties of Radiation in Fabry-Pérot Resonator}

Our consideration so far have applied to photons in an ideal spherical cavity. Consider now the very important case of interaction between a single atom with electric dipole transition and cavity field in the case of Fabry-Perot resonator formed by two parallel ideal reflecting mirrors. In this case, the cavity field can consist only of the photons propagating along the axis of resonator ( $z$ axis) because all other photons should leave the space limited by the mirrors. This means that the cavity photons have well-defined direction and therefore are in a state with given linear momentum (21)-(22). Hence, the radiation emitted by the electric dipole transition consists of the two modes with $m= \pm 1$, while the radiation of the third mode $m=0$ is forbidden. In this case, the photons with given helicity can be represented in terms of linearly polarized photons as follows [27];

$$
a_{\mu}=\mp \frac{a_{x} \pm i a_{y}}{\sqrt{2}}, \quad \mu= \pm 1
$$

Since the atom-field interaction in the Fabry-Perot resonator is allowed for the two electric dipole transitions

$$
|1, \pm 1\rangle \rightarrow|0,0\rangle
$$

the interaction term in the electric dipole Jaynes-Cummings Hamiltonian (34) takes the form

$$
H_{\mathrm{int}}=g \sum_{\mu= \pm 1}\left(R_{\mu g} a_{\mu}+a_{\mu}^{+} R_{g \mu}\right)
$$

Then, the representation of the $S U(2)$ algebra corresponding to the excited state in (105) takes the form

$$
\begin{aligned}
J_{z} & =\frac{\left.R_{++}-R_{+-}\right)}{2} \\
J_{+} & =R_{+-} \\
J_{-} & =R_{-+}
\end{aligned}
$$

similar to (50). Then, the exponential of the atomic $S U(2)$ phase operator takes the form

$$
\varepsilon=R_{+-}+e^{i \psi} R_{-+}
$$


such that

$$
\varepsilon \varepsilon^{+}=R_{++}+R_{--}=\mathbf{1}
$$

and

$$
\varepsilon^{2}=e^{i \psi} \mathbf{1}
$$

Here again $\psi$ denotes an arbitrary reference phase. The atomic cosine and sine of the phase operators (42) are defined in the case under consideration as follows:

$$
\begin{aligned}
& C=\left(R_{+-} e^{-i \psi / 2}+R_{-+} e^{i \psi / 2}\right) \cos \frac{\psi}{2}=\varepsilon e^{-i \psi / 2} \cos \frac{\psi}{2} \\
& S=\left(R_{+-} e^{-i \psi / 2}+R_{-+} e^{i \psi / 2}\right) \sin \frac{\psi}{2}=\varepsilon e^{-i \psi / 2} \sin \frac{\psi}{2}
\end{aligned}
$$

It is clear that these are commuting Hermitian operators.

In analogy to (45) and (46), we can define the atomic phase states as follows:

$$
\left|\phi_{\mu}\right\rangle=\frac{1}{\sqrt{2}}\left(|1,+1\rangle+e^{i \phi_{\mu}}|1,-1\rangle\right)
$$

where

$$
\phi_{\mu}=\frac{\psi+(1-\mu) \pi}{2}, \quad \mu= \pm 1
$$

It is seen that, apart from a factor of $e^{i \psi / 2}$, the states (110) formally coincide with the EPR states (49). It is clear that the operator (107) is represented in terms of states (109) in the diagonal form

$$
\varepsilon=\sum_{\mu= \pm 1} e^{i \phi_{\mu}}\left|\phi_{\mu}\right\rangle\left\langle\phi_{\mu}\right|
$$

[cf. (66)].

Following the approach discussed in Section IV.A, we note that the field counterpart of (106) is provided by the operators

$$
\begin{aligned}
& M_{z}=\frac{a_{+}^{+} a_{+}-a_{-}^{+} a_{-}}{2} \\
& M_{+}=a_{+}^{+} a_{-} \\
& M_{-}=a_{-}^{+} a_{+}
\end{aligned}
$$


which form the representation of the $S U(2)$ subalgebra in the Weyl-Heisenberg algebra of photons. As in (58), this is not an isotype representation. In analogy to (63), we introduce the field counterpart of (107)

$$
\varepsilon_{\mathrm{rad}}=a_{+}^{+} a_{-}+e^{i \psi} a_{-}^{+} a_{+}
$$

It is easy to verify that (113) complements the atomic operator (107) with respect to the integral of motion with the Jaynes-Cummings Hamiltonian (34) with the interaction term of the form (105). In analogy to (82), we introduce the cosine and sine of the radiation phase operators:

$$
\begin{aligned}
& C_{\mathrm{rad}}=K\left(\varepsilon_{\mathrm{rad}}^{+}+\varepsilon_{\mathrm{rad}}\right)=2 K e^{-i \psi / 2} \varepsilon_{\mathrm{rad}} \cos \frac{\psi}{2} \\
& S_{\mathrm{rad}}=i K\left(\varepsilon_{\mathrm{rad}}^{+}-\varepsilon_{\mathrm{rad}}\right)=2 K e^{-i \psi / 2} \varepsilon_{\mathrm{rad}} \sin \frac{\psi}{2}
\end{aligned}
$$

such that

$$
\left[C_{\text {rad }}, S_{\text {rad }}\right]=0
$$

and

$$
\left[\left(C+C_{\text {rad }}\right), H\right]=\left[\left(S+S_{\text {rad }}\right), H\right]=0
$$

Then, the condition (83) takes the form

$$
\left\langle C_{\mathrm{rad}}^{2}+S_{\mathrm{rad}}^{2}\right\rangle=4 K^{2} e^{-i \psi}\left\langle\varepsilon_{\mathrm{rad}}^{2}\right\rangle=1
$$

In analogy to (66), the operator (113) is diagonalized by the canonical transformation of the form

$$
\begin{aligned}
& a_{\mu}=\frac{1}{\sqrt{2}} \sum_{\mu^{\prime}= \pm 1} e^{-i\left(1-\mu^{\prime}\right) \phi_{\mu} / 2} \mathbf{a}_{\mu^{\prime}} \\
& \mathbf{a}_{\mu}=\frac{1}{\sqrt{2}} \sum_{\mu^{\prime}= \pm 1} e^{i\left(1-\mu^{\prime}\right) \phi_{\mu} / 2} a_{\mu}
\end{aligned}
$$

where $\phi_{\mu}$ is the phase variable (110).

Taking into account the equation (104), expressing the photons with given helicity in terms of linearly polarized photons, it is easily seen from (116) that we get

$$
\mathbf{a}_{+}=-a_{x} E^{i \psi / 2}, \quad \mathbf{a}_{-}=-i a_{y} e^{-i \psi / 2}
$$


Hence, the dual representation of the photon operators (104) with given helicity coincides, apart from certain unimportant factors, with the photon operators with given linear polarization. In the representation (116), the operators (113) and (114) take the diagonal form

$$
\begin{aligned}
\varepsilon_{\mathrm{rad}}^{(\phi)} & =\sum_{\mu= \pm 1} e^{i \phi_{\mu}} \mathbf{a}_{\mu}^{+} \mathbf{a}_{\mu} \cos \frac{\psi}{2} \\
C_{\mathrm{rad}}^{(\phi)} & =2 k \sum_{\mu= \pm 1} \mathbf{a}_{\mu}^{+} \mathbf{a}_{\mu} \cos \phi_{\mu} \cos \frac{\psi}{2} \\
S_{\mathrm{rad}}^{(\phi)} & =2 k \sum_{\mu= \pm 1} \mathbf{a}_{\mu}^{+} \mathbf{a}_{\mu} \sin \phi_{\mu} \cos \frac{\psi}{2}
\end{aligned}
$$

where the phase variable $\phi_{\mu}$ is defined in (110). Since the condition (115) can now be done in a straightforward manner to yield

$$
K=\frac{1}{2 \cos (\psi / 2) \sqrt{\left\langle\left(\mathbf{a}_{+}^{+} \mathbf{a}_{+}-\mathbf{a}_{-}^{+} \mathbf{a}_{-}\right)^{2}\right\rangle}}
$$

the expressions for cosine and sine operators in (117) take the form

$$
\begin{aligned}
C_{\mathrm{rad}}^{(\phi)} & =\frac{\sum_{\mu} \mathbf{a}_{\mu}^{+} \mathbf{a}_{\mu} \cos \phi_{\mu}}{\sqrt{\left\langle\left(\sum_{\mu} \mu \mathbf{a}_{\mu}^{+} \mathbf{a}_{\mu}\right)^{2}\right\rangle}} \\
S_{\mathrm{rad}}^{(\phi)} & =\frac{\sum_{\mu} \mathbf{a}_{\mu}^{+} \mathbf{a}_{\mu} \sin \phi_{\mu}}{\sqrt{\left\langle\left(\sum_{\mu} \mu \mathbf{a}_{\mu}^{+} \mathbf{a}_{\mu}\right)^{2}\right\rangle}}
\end{aligned}
$$

This representation explicitly manifests the structure of the cosine and sine operators in terms of cosine and sine of phase variable (110).

In analogy to (72) and (74) we now construct the dual Fock number states

$$
\begin{aligned}
\left|v_{\mu}\right\rangle & =\frac{\left(\mathbf{a}_{\mu}^{+}\right)^{v_{\mu}}}{\sqrt{v_{\mu} !}}|0\rangle \\
& =\sum_{k=0}^{v_{\mu}} \sqrt{\frac{v_{\mu} !}{2^{v_{\mu}} k !\left(v_{\mu}-k\right) !}}(-1)^{v_{\mu}-k}|k\rangle_{+} \otimes\left|v_{\mu}-k\right\rangle_{-}
\end{aligned}
$$

Here $v_{\mu}$ is an integer such that

$$
\mathbf{a}_{\mu}^{+} \mathbf{a}_{\mu}\left|v_{\mu}\right\rangle=v_{\mu}\left|v_{\mu}\right\rangle
$$


and the states in the right-hand side correspond to the photons with given helicity. It is easy to show that the states (120) obey the orthonormality and completeness conditions.

Following the method described in Section IV.C, we introduce the state

$$
\left|\phi^{(\mathrm{rad})}\right\rangle=\bigotimes_{\mu= \pm 1}\left|v_{\mu}\right\rangle
$$

[see (78)], which can be interpreted as the radiation phase state in the resonator under consideration. For a given total number of photons $n=\sum_{\mu} v_{\mu}$, there are $n+1$ different phase states with degenerated "eigenphase":

$$
\varphi_{n \ell}=\frac{\psi}{2}+2 \ell \pi, \quad \ell=1, \ldots, n+1
$$

This result is obtained by analogy with (79)-(81). Thus, unlike the case of spherical cavity, the spectrum of the $S U(2)$ phase of photons in the Fabry-Perot resonator is trivial.

To complete the comparison with the previous results obtained in Sections IV.C and IV.E, let us average the cosine operator in (114) over the states with two circularly polarized coherent modes $\left|\alpha_{+}, \alpha_{-}\right\rangle$. We get

$$
\left\langle C_{\mathrm{rad}}\right\rangle=\frac{2\left|\alpha_{+}\right|\left|\alpha_{-}\right| \cos \left(\Delta_{+-}+\psi / 2\right)}{\sqrt{\left|\alpha_{+}\right|^{2}+\left|\alpha_{-}\right|^{2}+4\left|\alpha_{+}\right|^{2}\left|\alpha_{-}\right|^{2} \cos ^{2}\left(\Delta_{+-}+\psi / 2\right)}} \cos \frac{\psi}{2}
$$

It is clear that

$$
\left\langle C_{\mathrm{rad}}\right\rangle \rightarrow 0
$$

in the "vacuum limit" $\left|\alpha_{ \pm}\right| \rightarrow 0$ independent of $\psi$, and

$$
\left\langle C_{\mathrm{rad}}\right\rangle \rightarrow \cos \frac{\psi}{2}
$$

in the classical limit $\left|\alpha_{ \pm}\right| \rightarrow \infty$. The behavior of corresponding variance is shown in Figs. 12 and 13 in the cases of $\left|\alpha_{+}\right|=\left|\alpha_{-}\right| \equiv|\alpha|$ and fixed $\left|\alpha_{-}\right|$ respectively. It is seen, that the variance shows the "normal" behavior and that the "phase bunching," discussed in Section IV.C, does not exist in the case under consideration. In contrast to (90), the presence of the component $\mu=-1$ in the quantum state $\left(\left|\alpha_{-}\right|^{2} \ll 1\right)$ leads to the decrease of fluctuations. 


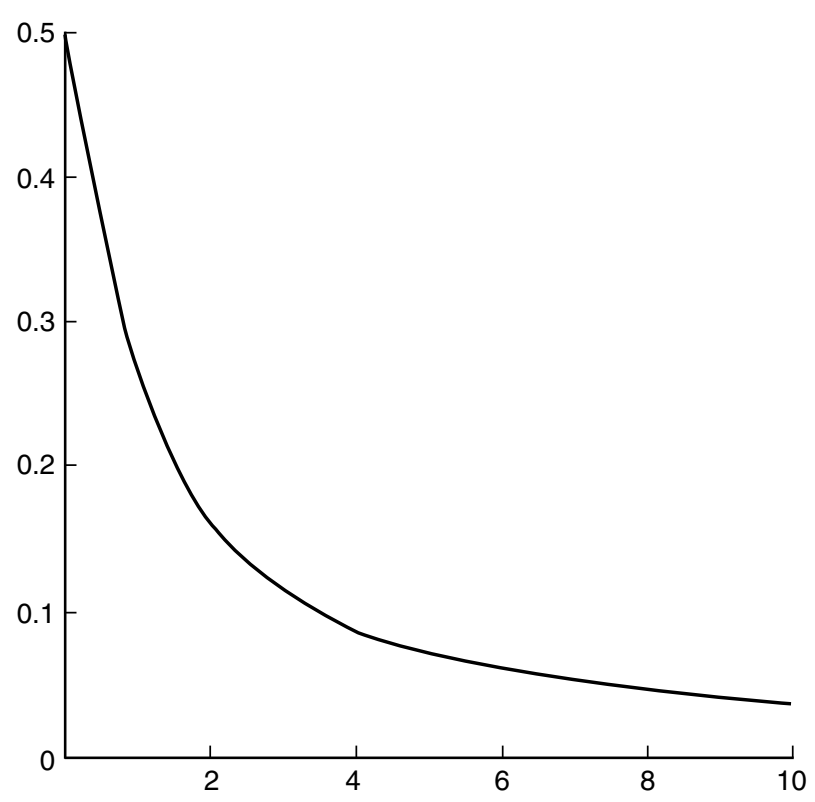

Figure 12. Variance of the cosine of radiation phase (119) in the two-mode coherent state at $\left|\alpha_{+}\right|^{2}=\left|\alpha_{-}\right|^{2}=|\alpha|^{2}$ versus $|\alpha|^{2}$.

\section{H. Summary}

1. There is no isotype representation of the $S U(2)$ subalgebra, describing the angular momentum of radiation, in the Weyl-Heisenberg algebra of photons. Therefore, the $S U(2)$ quantum phase of angular momentum of radiation field cannot be constructed in the same way as that of a quantum-mechanical system with a finite number of degrees of freedom.

2. At the same time, the conservation of angular momentum in the process of radiation makes it possible to map the atomic phase variable into the field variables via corresponding integrals of motion.

3. The Weyl-Heisenberg algebra of multipole photons allows the dual representation in which we deal with the photons with given radiation phase (the $S U(2)$ phase of angular momentum) instead of standard photons with given projection of the angular momentum.

4. Through the use of the dual representations of the atomic and field operators, it is possible to construct an equivalent form of the JaynesCummings Hamiltonian, describing the exchange of the "phase information" between the atom and radiation field. 


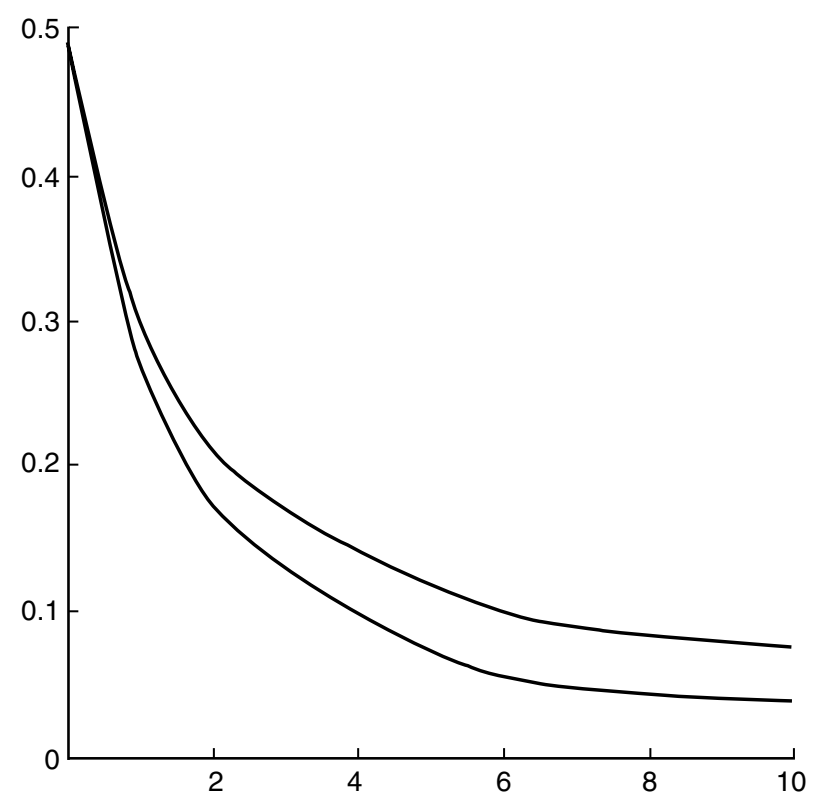

Figure 13. Variance of the cosine of radiation phase (119) in the two-mode coherent state as a function of $\left|\alpha_{+}\right|^{2}$ at fixed $\left|\alpha_{-}\right|^{2}=0.1$, respectively.

5. The radiation phase of multipole photons has discrete spectrum in the interval $(0,2 \pi)$. In the classical limit of high-intensity coherent field, the eigenvalues of the radiation phase are distributed uniformly over $(0,2 \pi)$.

6. The quantum fluctuations of the radiation phase manifest qualitative difference from those calculated within the Pegg-Barnett approach. In particular, the "phase bunching" effect can be observed for a multipole radiation in a spherical cavity in the quantum domain of low intensity. This effect does not occur in a linear cavity (Fabry-Perot resonator).

7. Our approach, leading to the definition of the radiation phase, is the natural complement of Mandel's operational approach. The radiation phase is defined in terms of what can be transmitted from a quantum mechanical system to photon and vice versa.

\section{POLARIZATION PROPERTIES OF MULTIPOLE RADIATION}

It is not really difficult to construct a series of inferences, each depends upon its predecessor and each simple in itself.

—Sir Arthur Conan Doyle, The Dancing Men 


\section{A. Polarization of Classical Field}

In previous sections, we considered the polarization as a formal property of either plane or spherical waves of photons described by the corresponding index in the expansion (13) and (17). In this Section, we examine the quantum properties of polarization in more details. In particular, we show that the radiation phase of electric dipole radiation formally coincides with the inherent quantum phase of polarization which is the $S U(2)$ phase of spin of photons.

It is well known that the polarization measurements play an important role in optics and spectroscopy [87]. The description usually given of the polarization is a classical one, defining the polarization as a measure of transversal anisotrophy of the plane electromagnetic waves [57]. It is based on the fact that the field strengths (11) have only two symmetric spatial components. At the same time, these complex components may have different magnitudes and phases. The quantitative description of polarization is provided by the so-called polarization matrix with the elements $[14,57]$

$$
P_{\sigma \sigma^{\prime}}=E_{\sigma}^{*}(\vec{r}) E_{\sigma^{\prime}}(\vec{r})
$$

Here $E_{\sigma}(\vec{r})$ denotes the component of the positive-frequency part of the classical electric field strength. For monochromatic plane waves, in view of (13) we get

$$
P_{\sigma \sigma^{\prime}}=\gamma^{2} a_{k \sigma}^{*} a_{k \sigma^{\prime}}
$$

By definition [14,25,57], the diagonal elements in (26) and (27) give the contribution of the corresponding spatial components of the radiation field into the energy density, while the off-diagonal elements give the "phase" information concerning the phase difference between the components.

The polarization matrix (124) can also be represented in the helicity basis (16) at $\vec{\chi}_{0}=\vec{k} / k$ as follows

$$
P_{\mu \mu^{\prime}}=\gamma^{2} a_{k \mu}^{*} a_{k \mu^{\prime}}
$$

where $\mu, \mu^{\prime}= \pm 1$ and

$$
a_{k \pm}=\mp \frac{a_{k x} \pm i a_{k y}}{\sqrt{2}}
$$

It is seen from (123)-(125) that $P$ is the Hermitian $(2 \times 2)$ matrix. In spite of the position dependence of the mode functions in (13), the elements of (124) and (125) have the same value everywhere. This means that the polarization is the global property of classical plane waves. 
In contrast to the plane waves, the field strengths of the multipole radiation can have any direction. In fact, the electric multipole radiation obey the condition $\overrightarrow{\mathscr{B}} \cdot \vec{r}=0$, while it can have nonzero longitudinal component $(\overrightarrow{\mathscr{E}} \cdot \vec{r} \neq 0)$ of the electric field strength [25]. In other words, this is the transversal magnetic radiation. In turn, the magnetic multipole field is characterized by the relations

$$
\overrightarrow{\mathscr{E}} \cdot \vec{r}=0, \quad \overrightarrow{\mathscr{B}} \cdot \vec{r} \neq 0
$$

Hence, the polarization of either multipole radiation should be specified by the spatial anisotrophy of the field strengths rather than the transversal anisotrophy as in the case of plane waves $[28,46,54,88]$. Thus, the polarization of the classical multipole field should be described by bilinear forms in all three components of the field strengths which leads to the Hermitian $(3 \times 3)$ matrix with the elements $[28,46]$

$$
P_{\mu \mu^{\prime}}^{(E)}(\vec{r})=E_{\mu}^{*}(\vec{r}) E_{\mu}(\vec{r}), \quad \mu, \mu^{\prime}= \pm 1,0
$$

Here we again consider a monochromatic radiation field. Let us stress that this expression describes the spatial anisotrophy of the electric field and therefore specifies the polarization of the electric multipole radiation. In the case of magnetic multipole radiation, the spatial anisotrophy of magnetic induction can be described by the following polarization matrix [89]:

$$
P_{\mu \mu^{\prime}}^{(M)}(\vec{r})=B_{\mu}^{*}(\vec{r}) B_{\mu^{\prime}}(\vec{r})
$$

Now consider the monochromatic multipole field with given $\lambda$ and $j$. Exactly this field is emitted by an atomic transition. Employing (21) and (17) then gives

$$
\begin{aligned}
P_{\mu \mu^{\prime}}^{(E)} & =k^{2} \sum_{m m^{\prime}} V_{E k j m \mu}^{*}(\vec{r}) V_{E k j m^{\prime} \mu^{\prime}}(\vec{r}) a_{E k j m \mu}^{*} a_{E k j m^{\prime} \mu^{\prime}} \\
P^{(M)} & =k^{2} \sum_{m m^{\prime}} V_{E k j m \mu}^{*}(\vec{r}) V_{E k j m^{\prime} \mu^{\prime}}(\vec{r}) a_{M k j m \mu}^{*} a_{M k j m^{\prime} \mu^{\prime}}
\end{aligned}
$$

Thus, the difference between $P^{(E)}$ and $P^{(M)}$ lies in the definition of the complex field amplitudes, while the position dependence is described in the same way. Within the classical picture, these amplitudes are defined differently in terms of the source functions [25]. For example, if the source of radiation is represented by the harmonically varying current $\vec{J}(\vec{r})$ and intrinsic magnetization $\overrightarrow{\mathscr{M}}(\vec{r})$, the field amplitudes for the electric radiation are determined by the integral of the function

$$
\left(\overrightarrow{\mathscr{M}}+\frac{1}{c k^{2}} \vec{\nabla} \times \vec{J}\right)
$$


over the volume of the source localization, while those for the magnetic radiation by the integrals of

$$
\left(\frac{\vec{J}}{c}+\vec{\nabla} \times \overrightarrow{\mathscr{M}}\right)
$$

It should be mentioned here that the quantum multipole radiation, defined in terms of the source, has also been considered [56].

In direct analogy to the case of plane waves, the diagonal elements of (129) describe the contribution of the components of the field strengths into the energy density. The off-diagonal elements give the "phase information" about the phase differences between the spatial components with different polarization. Unlike the plane waves, there are three phase differences $[46,54]$ :

$$
\Delta_{\mu \mu^{\prime}}(\vec{r})=\arg A_{E \mu}(\vec{r})-\arg A_{E \mu^{\prime}}(\vec{r})
$$

In view of the evident equality

$$
\Delta_{+0}+\Delta_{0-}+\Delta_{+}=0
$$

valid at any point $\vec{r}$, only two of the phase differences $\Delta_{\mu \mu^{\prime}}$ are independent.

In contrast to (124) and (125), the polarization matrix (129) of either multipole radiation depend on the position with respect to the source (origin). Since the component $A_{\mu=0}$ vanishes at far distance much faster than do the two transversal components, the polarization in the so-called far zone $(k r \gg 1)$ is similar to that of the plane waves.

In addition to the Hermitian polarization matrix with complex elements, the spatial anisotrophy of the electromagnetic field can be described by an equivalent set of real Stokes parameters [14,57].

The classical Stokes parameters of plane waves are usually defined in the linear polarization basis as follows: ${ }^{1}$

$$
\begin{aligned}
s_{0}^{\text {(plane) }} & =\left|\vec{e}_{x} \cdot \vec{E}\right|^{2}+\left|\vec{e}_{y} \cdot \vec{E}\right|^{2} \\
s_{1}^{\text {(plane) }} & =\left|\vec{e}_{x} \cdot \vec{E}\right|^{2}-\left|\vec{e}_{y} \cdot \vec{E}\right|^{2} \\
s_{2}^{\text {(plane) }} & =2 \operatorname{Re}\left[\left(\vec{e}_{x} \cdot \vec{E}^{*}\right)\left(\vec{e}_{y} \cdot \vec{E}\right)\right] \\
s_{3}^{\text {(plane) }} & =2 \operatorname{Im}\left[\left(\vec{e}_{x} \cdot \vec{E}^{*}\right)\left(\vec{e}_{y} \cdot \vec{E}\right)\right]
\end{aligned}
$$

${ }^{1}$ Unfortunately, there is no uniform notation for the Stokes parameters. Our notation is that of Ref. 57. 
In the helicity basis (16) we get

$$
\begin{aligned}
& s_{0}^{\text {(plane) }}=\left|E_{-}\right|^{2}+\left|E_{+}\right|^{2} \\
& s_{1}^{\text {(plane })}=-2 \operatorname{Re}\left(E_{+}^{*} E_{-}\right) \\
& s_{2}^{\text {(plane })}=2 \operatorname{Im}\left(E_{+}^{*} E_{-}\right) \\
& s_{3}^{\text {(plane })}=\left|E_{-}\right|^{2}-\left|E_{+}\right|^{2}
\end{aligned}
$$

where $E_{\mu} \equiv \vec{\chi}_{\mu} \cdot \vec{E}$. It is clear that the parameter $s_{0}^{\text {(plane) }}$ measures the relative intensity of the wave. At the same time, the expressions (130) and (131) show a rearrangement of the roles of the Stokes parameters with respect to the two bases. The parameter $s_{1}^{\text {(plane) }}$ in (130) gives the preponderance of the $x$-linear polarization over the $y$-linear polarization, while in (131) it concerns the phase difference between the two components with opposite helicities. The parameter $s_{2}^{\text {(plane) }}$ in (130) gives the cosine of the phase difference between the linearly polarized components, while in (131) it gives the sine of the phase difference between the circular polarized components. In turn, the parameter $s_{3}^{\text {(plane) }}$ in (48) specifies the sine of the phase difference in the Cartesian basis with linear polarizations, while in (131) it gives the preponderance of polarization with negative helicity over positive helicity.

Now consider the set of classical Stokes parameters of the monochromatic pure $(\lambda, j)$-pole radiation. Since the polarization, in this case, is described by the Hermitian $(3 \times 3)$ matrix $(127)$, the set should consist of nine Stokes parameters because all three spatial components of the field strength contribute into polarization [46]. For definiteness, let us consider the electric-type radiation. To establish a contact with the previous result (131), we choose the set of Stokes parameters as follows [46]:

$$
\begin{aligned}
& s_{0}^{(\text {multi) }}(\vec{r})=\sum_{\mu}\left|E_{\mu}\right|^{2} \\
& s_{1}^{\text {(multi) }}(\vec{r})=-2 \operatorname{Re}\left(E_{+}^{*} E_{0}+E_{0}^{*} E_{-}+E_{-}^{*} E_{+}\right) \\
& s_{2}^{\text {(multi) }}(\vec{r})=2 \operatorname{Im}\left(E_{+}^{*} E_{0}+E_{0}^{*} E_{-}+E_{-}^{*} E_{+}\right) \\
& s_{3}^{\text {(multi) }}(\vec{r})=\left|E_{-}\right|^{2}-\left|E_{+}\right|^{2} \\
& s_{4}^{\text {(multi) }}(\vec{r})=\left|E_{+}\right|^{2}+\left|E_{-}\right|^{2}-2\left|E_{0}\right|^{2} \\
& s_{5}^{(\text {multi) }}(\vec{r})=-2 \operatorname{Re}\left(E_{+}^{*} E_{-}\right) \\
& s_{6}^{(\text {multi) }}(\vec{r})=2 \operatorname{Im}\left(E_{+}^{*} E_{-}\right) \\
& s_{7}^{(\text {multi) }}(\vec{r})=-2 \operatorname{Re}\left(E_{0}^{*} E_{+}\right) \\
& s_{8}^{(\text {multi) }}(\vec{r})=2 \operatorname{Im}\left(E_{0}^{*} E_{+}\right)
\end{aligned}
$$


These parameters have very simple physical meanings. The parameter $s_{0}$ measures the contribution of all three components into the energy density. The parameters $s_{1,2,5,6,7,8}$ give the phase information about the phase differences between the circular and linear polarized components. The parameter $s_{3}$ gives the preponderance of negative helicity over positive helicity and the parameter $s_{4}$ gives the preponderance of transversal circular polarization over linear (radial) polarization. Unlike (131), the multipole Stokes parameters (132) describe the local properties of polarization due to the position dependence of the mode functions (18). It is clear that, at far distances $(k r \gg 1)$ when $E_{0}$ is negligibly small in comparison with $E_{ \pm}$, the set (132) formally coincides with (131) because

$$
\left\{\begin{array}{l}
s_{1}^{\text {(multi) }} \rightarrow s_{5}^{\text {(multi) }} \propto s_{1}^{\text {(plane) }} \\
s_{2}^{\text {(multi) }} \rightarrow s_{6}^{\text {(multi) }} \propto s_{2}^{\text {(plane) }} \\
s_{4}^{\text {(multi) }} \rightarrow s_{0}^{(\text {multi) }} \propto s_{0}^{\text {(plane) }}
\end{array}\right.
$$

and

$$
\left\{\begin{array}{l}
s_{7}^{(\text {multi })} \rightarrow 0 \\
s_{8}^{(\text {multi })} \rightarrow 0
\end{array}\right.
$$

in this limit.

\section{B. Polarization of Quantum Radiation}

The quantum counterpart of the polarization matrices can be constructed in direct analogy to the field quantization [90]. We have to subject the field amplitudes in (124), (125), and (129) to the Weyl-Heisenberg commutation relations (22) and (23) respectively. Thus, we get the operator matrices of polarization of the multipole radiation of the form

$$
\begin{aligned}
& \hat{P}_{\mu \mu^{\prime}}^{(E, n)}=k^{2} \sum_{m m^{\prime}} V_{E k j m \mu}^{*}(\vec{r}) V_{E k j m^{\prime} \mu^{\prime}}(\vec{r}) a_{E k j m \mu}^{+} a_{E k j m^{\prime} \mu^{\prime}} \\
& \hat{P}_{\mu \mu^{\prime}}^{(M, n)}=k^{2} \sum_{m m^{\prime}} V_{E k j m \mu}^{*}(\vec{r}) V_{E k j m^{\prime} \mu^{\prime}}(\vec{r}) a_{M k j m}^{+} a_{M k j m^{\prime}}
\end{aligned}
$$

These are the Hermitian $(3 \times 3)$ matrices with the operator elements defined in terms of the normal order of the creation and annihilation photon operators.

To clarify the structure of (133) and establish a contact with previous results for the radiation phase, consider the bare operator form of $\hat{P}^{(E, n)}$ in (133) in the case of the electric dipole radiation in a spherical cavity. The bare operator 
structure is provided by the limit $k r \rightarrow 0$ in the mode functions (18). This means that we consider the polarization of radiation directly near a source. Using the properties of spherical Bessel functions discussed in Section III.A, we get $[28,46]$

$$
\hat{P}_{m m^{\prime}}^{(E, n)}=\gamma_{E 1} a_{E k 1 m}^{+} a_{E k 1 m^{\prime}}
$$

where $\gamma_{E 1}$ is an unimportant normalization factor.

Now consider the set of Stokes operators that can be obtained by canonical quantization of (132). On the other hand, the Stokes operators should by definition represent the complete set of independent Hermitian bilinear forms in the photon operators of creation and annihilation. It is clear that such a set is represented by the generators of the $S U(3)$ subalgebra in the Weyl-Heisenberg algebra of electric dipole photons. The nine generators have the form [46]

$$
\begin{array}{ccc}
\left(a_{+}^{+} a_{+}-a_{0}^{+} a_{0}\right) & \left(a_{0}^{+} a_{0}-a_{-}^{+} a_{-}\right) & \left(a_{-}^{+} a_{-}-a_{+}^{+} a_{+}\right) \\
\frac{1}{2}\left(a_{+}^{+} a_{0}+a_{0}^{+} a_{+}\right) & \frac{1}{2}\left(a_{0}^{+} a_{-}+a_{-}^{+} a_{0}\right) & \frac{1}{2}\left(a_{-}^{+} a_{+}+a_{+}^{+} a_{-}\right) \\
\frac{1}{2 i}\left(a_{+}^{+} a_{0}-a_{0}^{+} a_{-}\right) & \frac{1}{2 i}\left(a_{0}^{+} a_{-}-a_{-}^{+} a_{0}\right) & \frac{1}{2 i}\left(a_{-}^{+} a_{+}-a_{+}^{+} a_{-}\right)
\end{array}
$$

and only eight of them are independent. To simplify the notations we omit here the indexes $E, k$, and $j=1$. To get the set of Stokes operators, we have to use the generators (135) or independent linear combinations of this generators together with the operator

$$
S_{0}=\sum_{m=-1}^{1} a_{m}^{+} a_{m}
$$

describing the total number of multipole photons. It seems to be logical to choose the rest of the set of Stokes operators as follows [46]

$$
\begin{aligned}
& S_{1}=\left(\varepsilon_{\mathrm{rad}}+\varepsilon_{\mathrm{rad}}^{+}\right) \\
& S_{2}=i\left(\varepsilon_{\mathrm{rad}}^{+}-\varepsilon_{\mathrm{rad}}\right) \\
& S_{3}=a_{+}^{+} a_{+}-a_{-}^{+} a_{-} \\
& S_{4}=a_{+}^{+} a_{+}+a_{-}^{+} a_{-}-2 a_{0}^{+} a_{0} \\
& S_{5}=\left(a_{+}^{+} a_{0}+a_{0}^{+} a_{+}\right) \\
& S_{6}=i\left(a_{0}^{+} a_{+}-a_{+}^{+} a_{0}\right) \\
& S_{7}=\left(a_{0}^{+} a_{-}+a_{-}^{+} a_{0}\right) \\
& S_{8}=i\left(a_{-}^{+} a_{0}-a_{0}^{+} a_{-}\right)
\end{aligned}
$$


Here the operator $\varepsilon_{\text {rad }}$ is as defined in (63). Thus, the operators $S_{1}$ and $S_{2}$ in (137) coincide, apart from a normalization factor, with the cosine and sine of the radiation phase operators (82). It seems to be natural. By construction, the operators $S_{1}$ and $S_{2}$ give the phase information about the phase differences $\Delta_{\mu \mu^{\prime}}$ defined in the previous subsection.

Additional phase information is provided by the operators $S_{6}-S_{8}$ in (137). To clarify the physical meaning of the phase-dependent operators in (137), let us average them over the two-mode coherent state (85) at $\left|\alpha_{+}\right|=\left|\alpha_{-}\right| \equiv|\alpha|$. We get

$$
\left\langle S_{\ell}\right\rangle=\left\{\begin{array}{lll}
2|\alpha|^{2} & \text { at } & \ell=0,3 \\
2|\alpha|^{2} \cos \Delta_{+-} & \text {at } & \ell=1 \\
2|\alpha|^{2} \sin \Delta_{+-} & \text {at } & \ell=2 \\
0 & \text { otherwise } &
\end{array}\right.
$$

Here

$$
\Delta_{+-} \equiv \arg \alpha_{+}-\arg \alpha_{-}
$$

as in Section IV.C. It is seen that the first two averages in (138) formally coincide with the conventional phase-dependent Stokes parameters defined in the helicity basis (131) [25,57]. Hence, the Stokes operators $S_{1}$ and $S_{2}$ give the cosine and sine of the radiation phase while the operators $S_{5}-S_{8}$ give the cosine and sine of the phase differences $\Delta_{0} \pm$ between the linear and one of the circular polarizations. Since

$$
\left[S_{1}, S_{2}\right]=0, \quad\left[S_{1}, S_{0}\right]=\left[S_{2}, S_{0}\right]=0
$$

the operators $S_{1}$ and $S_{2}$ form the Cartan algebra in the $S U(3)$ subalgebra (135) of the Weyl-Heisenberg algebra of multipole photons.

The remaining Stokes operators $S_{0}, S_{3}$, and $S_{4}$ in (133) and (134) also have simple physical meaning. In fact, $S_{0}$ describes the total number of photons (intensity), $S_{3}$ gives the preponderance of positive helicity over negative helicity, and $S_{4}$ defines the preponderance of transversal circular polarization over longitudinal linear polarization.

In spite of the formal coincidence between the Stokes parameters (138) and those obtained by quantization of (131) and further averaging over the coherent state (85), there is also a essential difference. Consider, for example, the variances of the Stokes operators $S_{1}$ and $S_{2}$ in (137):

$$
\begin{aligned}
& \left\langle\left(\Delta S_{1}\right)^{2}\right\rangle=2|\alpha|^{2}\left(2+\cos \Delta_{+-}\right) \\
& \left\langle\left(\Delta S_{2}\right)^{2}\right\rangle=2|\alpha|^{2}\left(2-\cos \Delta_{+-}\right)
\end{aligned}
$$


In turn, the variances of the corresponding Stokes operators describing the phase properties of plane waves of photons in the two-mode coherent state (85) have the form

$$
\left\langle\left(\Delta S_{1}\right)^{2}\right\rangle=\left\langle\left(\Delta S_{2}\right)^{2}\right\rangle=2|\alpha|^{2}
$$

Hence, the quantum fluctuations of the physical quantities, describing the phase information in the Stokes parameters are much stronger in the case of multipole radiation in comparison with the case of plane waves of photons. Moreover, they are qualitatively different because of the phase dependence in (140).

One more difference follows from the fact that, because of condition (139), the physical quantities described by $S_{1}$ and $S_{2}$ in (137) can be measured at once. At the same time, the operators, obtained by quantization of $S_{1}, S_{2}$, and $S_{3}$ in (131), form a representation of the $S U(2)$ algebra that excludes the possibility of simultaneous measurement of corresponding physical quantities.

\section{Spatial Properties of Polarization}

Our consideration of polarization so far has applied to the bare operator forms, corresponding to the normal-ordered polarization matrix and Stokes operators at the source location when $r \rightarrow 0$. In reality, (133) describes the positiondependent operator polarization matrix of the multipole radiation. In addition to the normal-ordered form of the operator polarization matrix (133), one can define the antinormal form:

$$
\begin{aligned}
& \hat{P}_{\mu \mu^{\prime}}^{(E, a n)}=k^{2} \sum_{m m^{\prime}} V_{E k j m \mu}^{*}(\vec{r}) V_{E k j m^{\prime} \mu^{\prime}}(\vec{r}) a_{E k j m^{\prime}} a_{E k j m}^{+} \\
& \hat{P}_{\mu \mu^{\prime}}^{(M, a n)}=k^{2} \sum_{m m^{\prime}} V_{E k j m \mu}^{*}(\vec{r}) V_{E k j m^{\prime} \mu^{\prime}}(\vec{r}) a_{M k j m^{\prime} \mu^{\prime}} a_{M k j m \mu}^{+}
\end{aligned}
$$

It is seen that, in view of the commutation relations (23), the difference

$$
P_{\mu \mu^{\prime}}^{(0)}(\vec{r})=\hat{P}_{\mu \mu^{\prime}}^{(\lambda, a n)}-\hat{P}_{\mu \mu^{\prime}}^{(\lambda, n)}=k^{2} \sum_{m} V_{E k j m \mu}^{*}(\vec{r}) V_{E k j m \mu^{\prime}}(\vec{r})
$$

defines the zero-point or vacuum oscillations of the components of the polarization of either multipole field [22,91]. In fact, the matrix elements of $P^{(0)}$ in (143) coincide with the commutators of the type

$$
\left[A_{\lambda k j m \mu}, A_{\lambda k j m^{\prime} \mu^{\prime}}^{+}\right]
$$


The trace of (143) coincides, apart from an unimportant factor, with the zeropoint fluctuations of energy (29).

It is seen from (143) that the vacuum polarization matrix is independent of index $\lambda$, describing the type of the multipole field. This seems to be natural, because the vacuum properties are affected by the presence of the singular point (atom) without respect to the type of radiation that might be emitted.

A similar object can be constructed in the case of plane waves of photons. In analogy to (133) and (142), we can construct the quantum counterpart of (123) and corresponding antinormal operator polarization matrix. Then, using the commutation relations (22) and definition (143), we get

$$
P_{\text {plane }}^{(0)}=\frac{2 \pi \hbar \omega_{k}}{V}\left(\begin{array}{ll}
1 & 0 \\
0 & 1
\end{array}\right)
$$

In contrast to (143), this is a diagonal matrix independent of the spatial variables. Hence, in exactly the same way as with the zero-point oscillations of energy density, the vacuum fluctuations of polarization in empty space has the global nature, while those in the presence of the singular point manifest certain spatial inhomogeneity.

It is intuitively clear that the spatial properties of the vacuum noise of polarization described by (143) should be determined by the distance $r$ from the source independently of the spherical angles $\theta$ and $\phi$. In fact, all directions from the singular point should be equivalent in the absence of radiation. It is possible to say that the multipole vacuum state is degenerated with respect to the directions from the source or is invariant under rotations about an arbitrary axis, passing through the source. This degeneration is offset by the generation of radiation of a given type $\lambda$ and with given $j$ and $m$, which causes the characteristic radiation pattern [25].

Consider first the polar direction when $\theta=0$ in (18). Then, due to the known property of spherical harmonics [70]

$$
Y_{j \pm 1, m-\mu}(0, \phi)=\sqrt{\frac{2(j \pm 1)+1}{4 \pi}} \delta_{m \mu}
$$

the mode functions in (19) are independent of the spherical angle $\phi$. Consider pure electric multipole radiation with given $j$. Then, the corresponding polarization matrix in (33) takes the form

$$
P_{\mu \mu^{\prime}}^{(E, n)}(r, 0, \phi)=k^{2} \mathscr{F}_{j \mu}^{*}(r) \mathscr{F}_{j \mu^{\prime}}(r) a_{E j \mu}^{+} a_{E j \mu^{\prime}}
$$


where

$$
\begin{aligned}
\mathscr{F}_{j \mu}(r) \equiv & V_{E j m \mu}(r, 0, \phi) \delta_{m \mu} \\
= & \sqrt{\frac{\hbar c}{2 k V(2 j+1)}\left[\sqrt{j(2 j+3)} f_{j+1}(k r)\langle 1, j+1, \mu, 0 \mid j \mu\rangle\right.} \\
& \left.-\sqrt{(j+1)(2 j-1)} f_{j-1}(k r)\langle 1, j-1, \mu, 0 \mid j \mu\rangle\right]
\end{aligned}
$$

It is seen that the photon operators with $|m| \geq 2$ do not contribute to the polarization of radiation in the polar direction even if $j \geq 2$. It is straightforward to calculate the elements of the vacuum polarization matrix (143) at $\theta=0$ :

$$
P_{\mu \mu^{\prime}}^{(0)}(r, 0, \phi)=k^{2} \sum_{j=1}^{\infty}\left|\mathscr{F}_{j \mu}^{*}(r)\right|^{2} \delta_{\mu \mu^{\prime}}
$$

Thus, the vacuum noise of polarization in the polar direction is represented by the diagonal matrix. Since the vacuum noise of polarization is supposed to be independent of the direction in the space, the matrix (143) can be put into the form (146) by a proper transformation of the reference frame spanned by the base vectors (16). We have

$$
U(\vec{r}) P^{(0)}(\vec{r}) U^{+}(\vec{r})=\mathscr{P}^{(0)}(r), \quad U^{+}(\vec{r}) U(\vec{r})=\mathbf{1}
$$

As a result of this transformation, $\vec{\chi}_{0}(\vec{r}) \rightarrow \vec{r} / r$. Here

$$
\mathscr{P}^{(0)}(r)=\left(\begin{array}{lll}
P_{T}(r) & 0 & 0 \\
0 & P_{L}(r) & 0 \\
0 & 0 & P_{T}(r)
\end{array}\right)
$$

and

$$
P_{T}(r)=k^{2} \sum_{j=1}^{\infty}\left|\mathscr{F}_{j \pm}(r)\right|^{2}, \quad P_{L}(r)=k^{2} \sum_{j=1}^{\infty}\left|\mathscr{F}_{j 0}(r)\right|^{2}
$$

The element $P_{T}$ describes the vacuum noise of transversal (with respect to $\vec{r}$ ) circular polarizations with positive and negative helicity, while $P_{L}$ gives the zero-point oscillations of linear polarization in the longitudinal direction (along $\vec{r})$. The explicit form of the unitary transformation (147) is

$$
U_{\mu \mu^{\prime}}=\frac{D_{\mu \mu^{\prime}}+\left(1-D_{\mu \mu}\right) \delta_{\mu \mu^{\prime}}}{\sqrt{1+\sum_{v \neq \mu}\left|D_{\mu \nu}\right|^{2}}}
$$


Here $D_{\mu \mu^{\prime}}(\vec{r})$ is expressed in terms of the elements of matrices (143) and (148) as follows

$$
\begin{aligned}
& D_{+0}=\frac{1}{F_{+}}\left[P_{--}^{(0)}\left(P_{T}-P_{++}^{(0)}\right)+\left.\left.\right|^{(0)} P_{+-}\right|^{2}\right] \\
& D_{+-}=-\frac{1}{F_{+}}\left[P_{0-}^{(0)}\left(P_{T}-P_{++}^{(0)}\right)+P_{+0}^{(0) *} P_{+-}^{(0)}\right] \\
& D_{0+}=\frac{1}{F_{0}}\left[P_{--}^{(0)}\left(P_{L}-P_{00}^{(0)}\right)+\left|P_{0-}^{(0)}\right|^{2}\right] \\
& D_{0-}=-\frac{1}{F_{0}}\left[P_{+-}^{(0)}\left(P_{L}-P_{00}^{(0)}\right)+P_{+0}^{(0)} P_{0-}^{(0)}\right] \\
& D_{-+}=\frac{1}{F_{-}}\left[P_{+0}^{(0) *}\left(P_{T}-P_{--}^{(0)}\right)+P_{0-}^{(0)} P_{+-}^{(0) *}\right] \\
& D_{-0}=-\frac{1}{F_{-}}\left[P_{++}^{(0)}\left(P_{T}-P_{--}^{(0)}\right)+\left|P_{+-}^{(0)}\right|^{2}\right]
\end{aligned}
$$

where

$$
\begin{aligned}
F_{+} & =\left(P_{+0}^{(0) *} P_{--}^{(0)}-P_{+-}^{(0) *} P_{0-}^{(0)}\right) \\
F_{0} & =\left(P_{+0}^{(0)} P_{--}^{(0)}-P_{0-}^{(0) *} P_{+-}^{(0)}\right) \\
F_{-} & =\left(P_{+-}^{(0)} P_{+0}^{(0) *}-P_{0-}^{(0)} P_{++}^{(0)}\right)
\end{aligned}
$$

It follows from the structure of (148) that the vacuum noise of transversal (with respect to $\vec{r}$ ) polarization described by $P_{T}$ is independent of helicity. At the same time, the transversal and longitudinal vacuum noises show different behavior as functions of distance (see discussion at the end of Section II).

To illustrate the spatial properties of the polarization of multipole radiation, consider the normal-ordered operator polarization matrix (133) in the case of monochromatic electric-type pure $j$-pole radiation. Assume that the radiation field is in a single-photon state $\left|1_{m}\right\rangle$ with given $m$. Then, the average of (133) takes the form

$$
\left\langle\hat{P}_{\mu \mu^{\prime}}^{(E, n)}(\vec{r})\right\rangle=k^{2} V_{E k j m \mu}^{*}(\vec{r}) V_{E k j m \mu^{\prime}}(\vec{r})
$$

It is seen that the state with given $m$ contributes to the polarization with different $\mu$. For variances of the elements of the polarization matrix, we get

$$
\begin{aligned}
\left\langle\left[\Delta \hat{P}_{\mu \mu^{\prime}}^{(E, n)}(\vec{r})\right]^{2}\right\rangle & \equiv\left\langle\hat{P}_{\mu \mu^{\prime}}^{(E, n) 2}\right\rangle-\left\langle\hat{P}_{\mu \mu^{\prime}}^{(E, n)}\right\rangle^{2} \\
& =k^{4} V_{E k j m \mu}^{*} V_{E k j m \mu^{\prime}} \sum_{m^{\prime} \neq m} V_{E k j m^{\prime} \mu}^{*} V_{E k j m^{\prime} \mu^{\prime}}
\end{aligned}
$$


It is also seen that the vacuum fluctuations of the field with $m^{\prime} \neq m$ contribute to the quantum noise of polarization of the mode with given $m$. Similar results can be obtained for the position dependent Stokes operators obtained from (132) by canonical quantization.

\section{Operator Polarization Matrix in the Proper Frame}

We saw that an appropriate choice of a local reference frame leads to the diagonal representation (148) of the vacuum polarization matrix (142). The use of the unitary transformation (147) allows the operator polarization matrix (142) to be cast into the form

$$
\mathscr{P}^{(E, n)}(\vec{r})=U(\vec{r}) P^{(E, n)}(\vec{r}) U^{+}(\vec{r})
$$

where

$$
\mathscr{P}_{\mu \mu^{\prime}}^{(E, n)}(\vec{r})=k^{2} \mathscr{A}_{E k j \mu}^{+}(\vec{r}) \mathscr{A}_{E k j \mu^{\prime}}(\vec{r})
$$

and

$$
\mathscr{A}_{E k j \mu}(\vec{r})=\sum_{\mu^{\prime}=-1}^{1} U_{\mu \mu^{\prime}}^{*}(\vec{r}) \sum_{m=-j}^{j} V_{E k j m \mu^{\prime}}(\vec{r}) a_{E k j m}
$$

Similar representation can be constructed for the magnetic-type operator polarization matrix in (142) as well.

It is clear that, in view of (22), the operators (152) obey the commutation relations

$$
\left[\mathscr{A}_{\lambda k j \mu}(\vec{r}), \mathscr{A}_{\lambda^{\prime} k^{\prime} j^{\prime} \mu^{\prime}}^{+}(\vec{r})\right]=\delta_{\lambda \lambda^{\prime}} \delta_{k k^{\prime}} \delta_{j j^{\prime}} \delta_{\mu \mu^{\prime}} \times\left\{\begin{array}{lll}
P_{T}(r) & \text { at } & \mu= \pm 1 \\
P_{L}(r) & \text { at } & \mu=0
\end{array}\right.
$$

Here $P_{\mu= \pm} \equiv P_{T}$ and $P_{\mu=0} \equiv P_{L}$ are the matrix elements of the diagonal vacuum polarization matrix (148). The representation of the Stokes operators in the proper frame can be constructed in the same way.

We now note that the only difference between (153) and commutation relations (23) is the presence of position-dependent factors in the right-hand side of (153). It seems to be quite tempting to introduce the normalized local operators

$$
b_{\lambda k j \mu}(\vec{r})=\frac{\mathscr{A}_{\lambda k j \mu}(\vec{r})}{\sqrt{P_{\mu}(\vec{r})}}
$$


which obey the standard Weyl-Heisenberg commutation relations

$$
\left[b_{\lambda k j \mu}(\vec{r}), b_{\lambda^{\prime} k^{\prime} j^{\prime} \mu^{\prime}}^{+}(\vec{r})\right]=\delta_{\lambda \lambda^{\prime}} \delta_{k k^{\prime}} \delta_{j j^{\prime}} \delta_{\mu \mu^{\prime}}
$$

at any point $\vec{r}$. Hence, the local transformation (152), representing the components of the operator vector potential in the proper frame, can be interpreted as a local Bogolubov canonical transformation [80], conserving the commutation relations. In fact, Eqs. (152) and (154) describe the transformation of global multipole photon operators $a_{\lambda k j m}$ with given $m=-j, \ldots, j$ into the local photon operators $b_{\lambda k j \mu}(\vec{r})$ with given polarization $\mu=0, \pm 1$ at any point of the space.

Due to the form of the operator polarization matrix (142) and corresponding Stokes operators, the polarization, defined to be the spin state of photons [4,27], is not a global property of the quantum multipole radiation. Any atomic transition emits photons with given quantum number $m$, which yields, in view of (18), (24), and (142), the polarization of all three types depending on the distance from the atom. The structure of (152) and (154) just shows us how the photons with different $m$ contribute into the polarization at an arbitrary point $\vec{r}$. Using the operators (154), we can construct, for example, the local bare operator representation of the polarization matrix (142) as follows

$$
p_{\mu \mu^{\prime}}^{(E, n)}(\vec{r})=b_{E k j \mu}^{+}(\vec{r}) b_{E k j \mu^{\prime}}(\vec{r})
$$

as well as of the Stokes operators: ${ }^{2}$

$$
\begin{aligned}
& S_{0}(\vec{r})=\sum_{\mu} b_{\mu}^{+}(\vec{r}) b_{\mu}(\vec{r}) \\
& S_{1}(\vec{r})=\bar{\varepsilon}(\vec{r})+\bar{\varepsilon}^{+}(\vec{r}) \\
& S_{2}(\vec{r})=-i\left(\bar{\varepsilon}(\vec{r})-\bar{\varepsilon}^{+}(\vec{r})\right) \\
& S_{3}(\vec{r})=b_{+}^{+}(\vec{r}) b_{+}(\vec{r})-b_{-}^{+}(\vec{r}) b_{-}(\vec{r}) \\
& S_{4}(\vec{r})=b_{+}^{+}(\vec{r}) b_{+}(\vec{r})+b_{-}^{+}(\vec{r}) b_{-}(\vec{r})-2 b_{0}^{+}(\vec{r}) b_{0}(\vec{r}) \\
& S_{5}(\vec{r})=\left(b_{+}^{+}(\vec{r}) b_{0}(\vec{r})+b_{0}^{+}(\vec{r}) b_{+}(\vec{r})\right) \\
& S_{6}(\vec{r})=-i\left(b_{+}^{+}(\vec{r}) b_{0}(\vec{r})-b_{0}^{+}(\vec{r}) b_{+}(\vec{r})\right) \\
& S_{7}(\vec{r})=\left(b_{0}^{+}(\vec{r}) b_{-}(\vec{r})+b_{-}^{+}(\vec{r}) b_{0}(\vec{r})\right) \\
& S_{8}(\vec{r})=-i\left(b_{0}^{+}(\vec{r}) b_{-}(\vec{r})-b_{-}^{+}(\vec{r}) b_{0}(\vec{r})\right)
\end{aligned}
$$

where

$$
\bar{\varepsilon}(\vec{r}) \equiv b_{+}^{+}(\vec{r}) b_{0}(\vec{r})+b_{0}^{+}(\vec{r}) b_{-}(\vec{r})+b_{-}^{+}(\vec{r}) b_{+}(\vec{r})
$$

${ }^{2}$ Hereafter we omit all unimportant indices. 
It is seen that (157) has the operator structure and algebraic properties similar to those of (136)-(137). At $r \rightarrow 0$, the set (157) exactly coincides with (136)(137). Due to the commutation relations (155), the operators (157) have the same algebraic properties as do (136)-(137) at any given point. In particular, we can construct the local representation of the radiation phase operators in the same way as in Section IV, using the operator (158) instead of (63). By construction, this gives us the $S U(2)$ quantum phase of spin or polarization with the properties described in Section IV.C.

Let us stress a very important difference between the representations of Stokes operators (137) and (157). If the former is valid only for the electric dipole photons, the latter describes an arbitrary multipole radiation with any $\lambda$ and $j$. The similarity in the operator structure and quantum phase properties is caused by the same number of degrees of freedom defining the representation of the $S U(2)$ subalgebra in the Weyl-Heisenberg algebra.

\section{E. Summary}

1. The polarization is described by a bilinear forms in the field components corresponding to the spin states of photons. In general, the polarization is defined by nine physical parameters (operators, in the quantum picture). In the case of plane waves of photons when only two spin states are allowed, the polarization is specified by only four parameters (operators).

2. The set of Stokes operators in the general case corresponds to a representation of the $S U(3)$ subalgebra in the Weyl-Heisenberg algebra of photons. In the case of plane waves of photons, it reduces to the representation of the $S U(2)$ subalgebra.

3. The $S U(2)$ quantum phase of spin (polarization) of photons described in a proper reference frame coincides with the radiation phase of electric dipole radiation discussed in Section IV.

\section{MEASUREMENT, LOCALITY, AND CAUSALITY}

And then he would have lost sight of the mark he had made on the wall, where the nail was to go in, and each had to get up on the chair, besides him, and see if we could find it; and we would each discover it in a different place, and he would call us all fools, one after another, and tell us to get down. And he would take the rule, and re-measure, and find that he wanted half thirty one and three-eights inches from the corner, and try to do it in his head, and go mad.

-Jerome K. Jerome, Three Men in a Boat

\section{A. Measurement and Photon Localization}

It was indicated in Section I that, although the photon operators refer to the radiation field in all space, it looks tempting to interpret the electronic signal 
registered by a photodetector as due to a photon localization in a vicinity of the sensitive area of this detector. The corresponding operational definition of localization has been done by Mandel [20] (see also Refs. 14, 15, and 92). It is based on the consideration of a plane wave of photons, being absorbed by the sensitive area $s$ of photodetector during some finite time $\Delta \tau$. Then, it is logical to interpret this process as a measurement of photons located in a cylindrical volume $\mathscr{V}=s \cdot(\Delta \tau)$ (Fig. 14).

Following [14], we introduce a photon absorption operator at the point $\vec{r}$ at time $t$ as follows:

$$
\vec{a}(\vec{r}, t)=\gamma \sum_{k, \sigma}{ }^{\prime} \vec{e}_{k \sigma} a_{k \sigma} e^{i(\vec{k} \cdot \vec{r}-k c t)}
$$

Here $\gamma$ is the normalization factor, $a_{k \sigma}$ are the operators (21)-(22), and summation is taken over a finite set of modes to which the detector responds. The socalled configuration space number operator [14] is defined by the relation

$$
\begin{aligned}
\mathscr{N}(\mathscr{V}, t) & =\int_{\mathscr{V}} \vec{a}^{\prime}(\vec{r}, t) \cdot \vec{a}(\vec{r}, t) d^{3} r \\
& =\gamma^{2} \sum_{k, \sigma} \sum_{k^{\prime}, \sigma^{\prime}} \vec{e}_{k \sigma} \cdot \vec{e}_{k^{\prime} \sigma^{\prime}} e^{-i\left(\vec{k}-\vec{k}^{\prime}\right) \cdot \vec{r}} e^{i\left(k-k^{\prime}\right) c t} a_{k \sigma}^{+} a_{k^{\prime} \sigma^{\prime}}
\end{aligned}
$$

where the integral is taken over the volume of photon localization. The operators (159) and (160) obey the following commutation relations [14]

$$
\left[\mathscr{N}(\mathscr{V}, t), \mathscr{N}\left(\mathscr{V}^{\prime}, t\right)\right]=0
$$
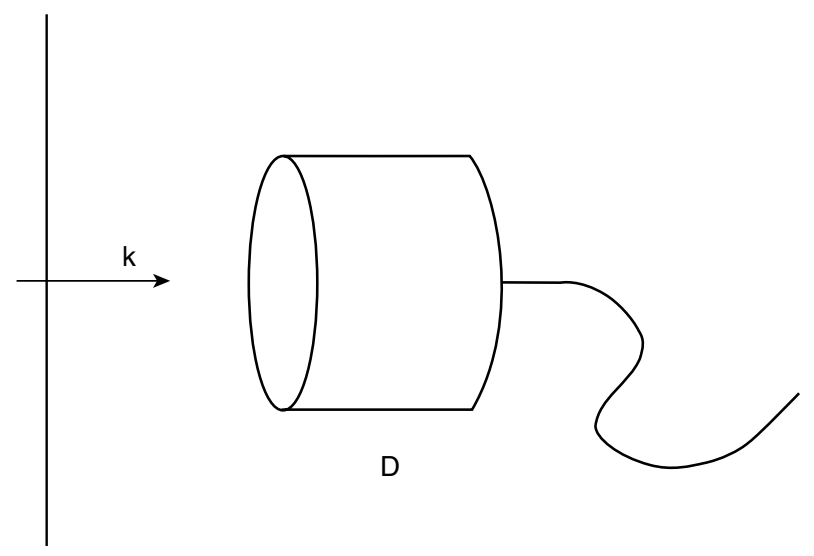

Figure 14. Photodetection and region of localization. 
and

$$
[\vec{a}(\vec{r}, t), \mathscr{N}(\mathscr{V}, t)] \approx\left\{\begin{array}{lll}
-\vec{a}(\vec{r}, t) & \text { if } & \vec{r} \in \mathscr{V} \\
0 & \text { otherwise } &
\end{array}\right.
$$

Let us stress that (162) has an approximate sense.

There is a principal difference that complicates the direct use of the operation approach to the problem of localizing photons in the case of multipole radiation. The point is that the multipole photons are in a state with given angular momentum and therefore they have no well-defined direction of propagation. In view of the wave-particle dualism, one can say that the multipole photons emitted by a point-like quantum source propagate as outgoing spherical waves. Definitely, these photons are localized initially inside the source.

Consider a model of Hertz-type experiment on emission and detection of a multipole photon in the system of two identical atoms separated by a distance $d$. If we assume that a photon is first emitted by the atom 1 (source) and then absorbed by the atom 2 (detector), it is most natural to consider the field as a superposition of outgoing and incoming spherical waves focused on the source and detector, respectively (Fig. 15). This superposition should obey the boundary conditions for the real radiation field. Then, in analogy to (159) and (160), one can construct a configuration space photon number operator via the integration of the corresponding local operator over the spherical volume of radius $c \Delta \tau$, surrounding the detecting atom. Here $\Delta \tau$ is again the detection time.

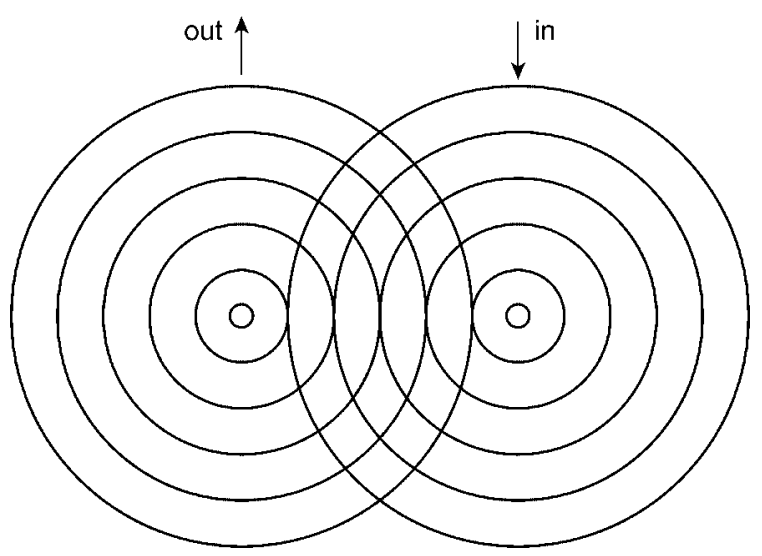

Figure 15. The scheme of two-atom Hertz experiment. 
Considering into account that (159) is simply the positive-frequency part of the vector potential (21), we can introduce the multipole photon absorption operator as follows

$$
\overrightarrow{\mathbf{a}}(\vec{r}, t)=\sum_{\mu}(-1)^{\mu} \vec{\chi}_{-\mu} \sum_{\lambda k j m}^{\prime} V_{\lambda k j m \mu}(\vec{r}) e^{-i k c} a_{\lambda k j m}
$$

which coincides with (24) apart from the fact that the sum is taken over the modes allowed by the selection rules. Here the mode function is defined by Eq. (18) with the radial part (19) corresponding to the incoming spherical wave. Then, the configuration space multipole photon number operator takes the form

$$
\mathbf{N}(\mathscr{V}, t)=\int_{\mathscr{V}} \overrightarrow{\mathbf{a}}^{+}(\vec{r}, t) \cdot \overrightarrow{\mathbf{a}}(\vec{r}, t) d^{3} r
$$

similar to (162). Here the volume of detection is

$$
\mathscr{V}=\frac{4 \pi}{3}\left[(c \Delta \tau)^{3}-r_{a}^{3}\right]
$$

where $r_{a}$ is the atomic radius. We have to exclude the "volume of generation" occupied by the atom to avoid the divergence at $r \rightarrow 0$ in the case of outgoing and incoming spherical waves of photons. By virtue of the transformation (152), we can rewrite it as

$$
\mathbf{N}(\mathscr{V}, t)=\int_{\mathscr{V}} \overrightarrow{\mathscr{A}}^{+}(\vec{r}, t) \cdot \overrightarrow{\mathscr{A}}(\vec{r}, t) d^{3} r
$$

where the definition of the components of $\overrightarrow{\mathscr{A}}$ differs from (152) by summation over all allowed modes.

Taking into account the properties of spherical harmonics [70], ClebschGordon coefficients [71], and spherical Bessel and Hankel functions [70], it is possible to show that the mode functions in (18) obey the following condition of symmetry:

$$
V_{\lambda k j,-m,-\mu}(\vec{r})=(-1)^{m+j-\mu} V_{\lambda k j m \mu}^{*}(\vec{r})
$$

Then, it can be easily seen that the commutation relation (161) is valid for the operators $\mathbf{N}$ as well:

$$
\left[\mathbf{N}(\mathscr{V}, t), \mathbf{N}\left(\mathscr{V}^{\prime}, t\right)\right]=0
$$


The commutation relation corresponding to (162) is less simple. It can be proven as an approximate one.

Thus, the picture of measurement in the atom-detector system of two identical atoms is compatible with Mandel's operational approach to the photon localization. For example, the multipole photon statistics in finite volume can be examined in the same way as in Refs. 14 and 20. The commutators for different $t$ can also be constructed in analogy to Ref. 20.

Nevertheless, there is one important difference. The point is that the zeropoint oscillations of the multipole field are concentrated in a vicinity of the atoms, where they can strongly exceed the level calculated in the model of plane waves in empty space (see Section II.B). If the atomic separation is large in comparison to the wavelength, then a major contribution into the vacuum noise of measurement comes from the presence of the detecting atom. At the intermediate and short distances, the vacuum noise in the vicinity of the detecting atom is increased because of the influence of the source atom via the superposed zero-point oscillations of outgoing and incoming spherical waves. Since the vacuum noise influences the precision of measurements $[14,15,58,59,86]$, this fact seems to be very important, especially for the experiments with trapped Ridberg atoms, which are usually separated by distances corresponding to the intermediate and even near zone $[32,66]$.

Now consider the measurement of monochromatic plane photons by a photodetector shown in Fig. 14. At far distances, the photons are specified by a unique wave vector $\vec{k}$. Mandel's localization of photons in the vicinity of the sensitive area $\sigma$ assumes that the wave converges to $\sigma$. This means that there is a variety of directions of the wavevectors near $\sigma$ (Fig. 16). This picture can be described by a proper expansion over spherical waves. In view of the discussion

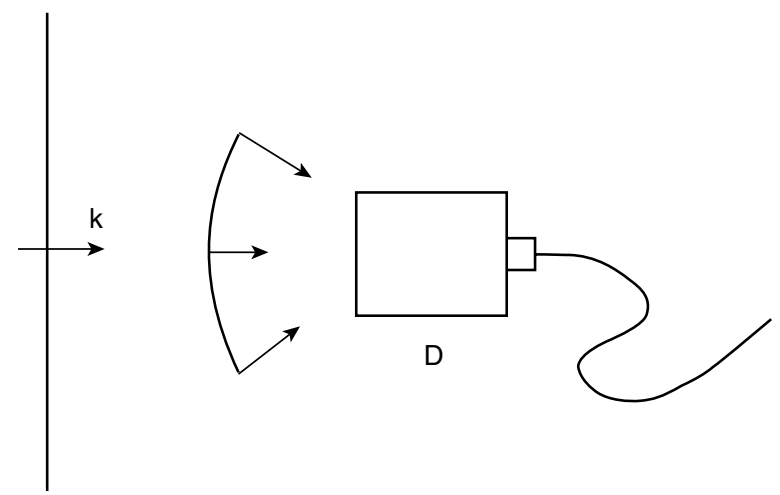

Figure 16. Absorption of radiation by photodetector. 
above, it should lead to an increase of the vacuum noise of measurement over the level of plane waves as well.

\section{B. Causality in the Two-Atom Hertz Experiment}

In the previous subsection, we considered the field emitted by one atom and then absorbed by another atom as a superposition of outgoing and incoming spherical waves of multipole photons. This wave picture completely eliminates an inquiry concerning the trajectory of photons between the atoms. In fact, the path of a particle in quantum mechanics is not a well-defined notion. The most that we can state about the path of a quantum particle in many cases is that it is represented by a nondifferentiable, statistically self-similar curve [93]. For example, the path of a tunneling electron and time spending in the barrier are not still defined unambiguously [94]. Moreover, some experiments on photonic tunneling and transmission of information show the possibility of superluminal motion of photons inside an opaque barrier [95].

We now note that, according to the principles of quantum theory, not the path, but causality in the transmission of information from one object to another is important [3,10-13,31]. In the Hertz experiment with two atoms separated by empty space, this means that the detecting atom cannot be excited earlier than in $d / c$ seconds after the emission of a photon by the first atom. Here $d$ denotes the interatomic distance. Such a causality has been proven recently by Kaup and Rupasov [96]. Here we briefly discuss their proof.

In the model experiment under consideration, the field is represented by the outgoing and incoming spherical waves of photons, which are specified by a continuous distribution of $k$ or of $\omega=c k$. Assume that the two identical atoms are the two-level atoms of the type of (34) with the electric dipole transition. Because of the simple geometry of the problem (Fig. 17), it can be considered as a quasiunidimensional integrable system [69]. The effective spatial dependence of the photon operators can be introduced with the aid of the Fourier transformation

$$
c_{m}(x)=\int_{-\infty}^{\infty} \frac{d \omega}{2 \pi} e^{i\left(\omega-\omega_{0}\right) x} a_{m}(\omega)
$$

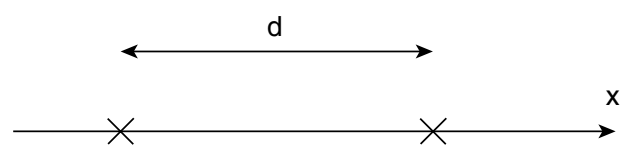

Figure 17. Effective geometry of the model. The $x$ axis corresponds to the quasi-onedimensional time-dependent wavefunction. 
where $a_{m}(\omega) \equiv a_{E 1 \mathrm{~km}}$ and $\omega_{0}$ is the frequency of the atomic transition. According to (23), the operators obey the commutation relations

$$
\left[c_{m}(x), c_{m^{\prime}}^{+}\left(x^{\prime}\right)\right]=\delta_{m m^{\prime}} \delta\left(x-x^{\prime}\right)
$$

In analogy to (34), the Hamiltonian of the system under consideration can be represented as follows:

$$
\begin{aligned}
H & =H_{0}+H_{\mathrm{int}}+\omega_{0} N \\
H_{0} & =-i \sum_{m} \int_{-\infty}^{\infty} c_{m}^{+}(x)\left(\frac{d}{d x} c_{m}(x)\right) d x \\
H_{\mathrm{int}} & =\sqrt{\eta} \sum_{m} \sum_{f=1,2} \int_{-\infty}^{\infty}\left[R_{m g}^{(f)} c_{m}(x)+c_{m}^{+}(x) R_{g m}^{(f)}\right] \delta\left(x-x_{f}\right) d x \\
N & =\sum_{m}\left[\sum_{f} R_{m m}^{(f)}+\int_{-\infty}^{\infty} c_{m}^{+}(x) c_{m}(x) d x\right]
\end{aligned}
$$

Here $\sqrt{\eta}$ denotes the coupling constant, index $f=1,2$ denotes the atoms, $x_{f}$ is the atomic position along the $x$ axis (see Fig. 17), and the operator $N$ describes the total number of excitations in the system. It is clear that

$$
[N, H]=0
$$

Using the notation of Section III.A, we define the ground state of the system

$$
\mid \text { Ground }\rangle=\left|j^{\prime}=0, m^{\prime}=0\right\rangle_{f=1} \otimes\left|j^{\prime}=0, m^{\prime}=0\right\rangle_{f=2} \otimes|0\rangle
$$

as the state of two unexcited atoms and vacuum field.

Assume that the first atom is initially excited into one of the sublevels of the excited state with a given probability:

$$
\begin{aligned}
\mid \text { in }\rangle & \left.=\sum_{m} p_{m} R_{m g}^{(1)} \mid \text { ground }\right\rangle \\
\sum_{m}\left|p_{m}\right|^{2} & =1
\end{aligned}
$$

Then, the Schrödinger time evolution is described by the wavefunction

$$
\left.|\Psi(t)\rangle=e^{-i H t} \mid \text { in }\right\rangle
$$


Let us denote by $|\zeta\rangle$ the eigenstates of the Hamiltonian (166):

$$
H|\zeta\rangle=E|\zeta\rangle
$$

Here, in general, $\zeta$ is a complex parameter. Then, the wavefunction (169) can be represented in the following way:

$$
\left.|\Psi(t)\rangle=\int_{-\infty}^{\infty} \frac{d \zeta}{2 \pi} L(\zeta) e^{-i E t} \mid \text { in }\right\rangle
$$

To find the eigenstates $|\zeta\rangle$ and eigenvalues $E$ in (170), we now note that, because of the initial condition (168), the states with single excitation only are allowed in the system. These states, in general, can be chosen as follows:

$$
\left.|\zeta\rangle=\sum_{m}\left[\sum_{f} \xi_{m}^{(f)}(\zeta) R_{m g}^{(f)}+\int_{-\infty}^{\infty} e^{i \zeta x} f_{m}(\zeta, x) c_{m}^{+}(x) d x\right] \mid \text { ground }\right\rangle
$$

Employing (170) then gives the following set of equations for unknown functions $f_{m}(\zeta, x)$ and $\xi_{m}^{(f)}(\zeta)$ :

$$
\begin{aligned}
i \frac{d}{d x}\left[e^{i \zeta x} f_{m}(\zeta, x)\right]+E e^{i \zeta x} f_{m}(\zeta, x) & =\sqrt{\eta} \sum_{f} \xi_{m}^{(f)} \delta\left(x-x_{f}\right) \\
\sqrt{\eta} \int e^{i \zeta x} f_{m}(\zeta, x) \delta\left(x-x_{f}\right) & =E \xi_{m}^{(f)}(\zeta)
\end{aligned}
$$

It is seen that the first equation in (173) gives formally a nonphysical discontinuity at $x \rightarrow x_{f}$. To avoid this, we have to consider the finite-size atoms and change $\delta\left(x-x_{f}\right)$ by a smooth distribution function $u\left(x, x_{f}\right)$, then solve the equations, and after that to put $u\left(x, x_{f}\right)=\delta\left(x-x_{f}\right)$. We get

$$
\begin{aligned}
f_{m}(\zeta, x) & =\prod_{f=1,2} \frac{\zeta-i(\eta / 2) \operatorname{sgn}\left(x-x_{f}\right)}{\zeta+i \eta / 2} \\
\xi_{m}^{(1)} & =\frac{\sqrt{\eta}}{\zeta+i \eta / 2} e^{i \zeta x_{1}} \\
\xi_{m}^{(2)} & =\frac{\zeta-i \eta / 2}{\zeta+i \eta / 2} \frac{\sqrt{\eta}}{\zeta+i \eta / 2} e^{i \zeta x_{2}}
\end{aligned}
$$

where

$$
\operatorname{sgn}\left(x-x_{f}\right)=\left\{\begin{array}{ccc}
1 & \text { at } & x>x_{f} \\
-1 & \text { at } & x<x_{f} \\
0 & \text { at } & x=x_{f}
\end{array}\right.
$$

and $E=\zeta$, which is real. Thus, the function (172) is completely defined. 
Since any two states (172) obey the orthogonality condition

$$
\left\langle\zeta \mid \zeta^{\prime}\right\rangle=2 \pi \delta\left(\zeta-\zeta^{\prime}\right)
$$

and, in view of (171) the initial state can be expanded in the following way

$$
\mid \text { in }\rangle=\int \frac{d \zeta}{2 \pi} L(\zeta)|\zeta\rangle
$$

and for the coefficients in (172) we get

$$
L(\zeta)=\langle\zeta| \text { in }\rangle=\frac{\sqrt{\eta}}{\zeta-i \eta / 2}
$$

Finally, for the function (172), describing the time evolution of the system, we get

$$
|\Psi(t)\rangle=\sum_{m} p_{m} \int_{-\infty}^{\infty} \frac{d \zeta}{2 \pi} \frac{\sqrt{\eta}}{\zeta-i \eta / 2} e^{-i \zeta t}|\zeta\rangle
$$

This wavefunction can be used to calculate the evolution of any physical quantity in the system under consideration. To prove the causality, we now have to calculate the time-dependent expectation value

$$
\left\langle R_{m m}^{(2)}\right\rangle_{t}=\left|p_{m}\right|^{2}\left|\int \frac{d \zeta}{2 \pi} \frac{\eta}{(\zeta+i \eta / 2)^{2}} e^{-i \zeta(t-d / c)}\right|^{2}
$$

describing the evolution of population of corresponding sublevel of the detecting atom. It is now a straightforward matter to arrive at the following result

$$
\left\langle R_{m m}^{(2)}\right\rangle=\left|p_{m}\right|^{2} \times \begin{cases}0 & 0 \leq t \leq \frac{d}{c} \\ \eta^{2}\left(t-\frac{d}{c}\right)^{2} e^{-\sqrt{\eta}(t-d / c)} & t>\frac{d}{c}\end{cases}
$$

(see Fig. 18). It is seen that this average shows the causal behavior.

\section{Polarization Measurements}

Instead of discussing the well-known methods of polarization measurements [87], we now turn our attention to the fluctuations in the measurement of the 


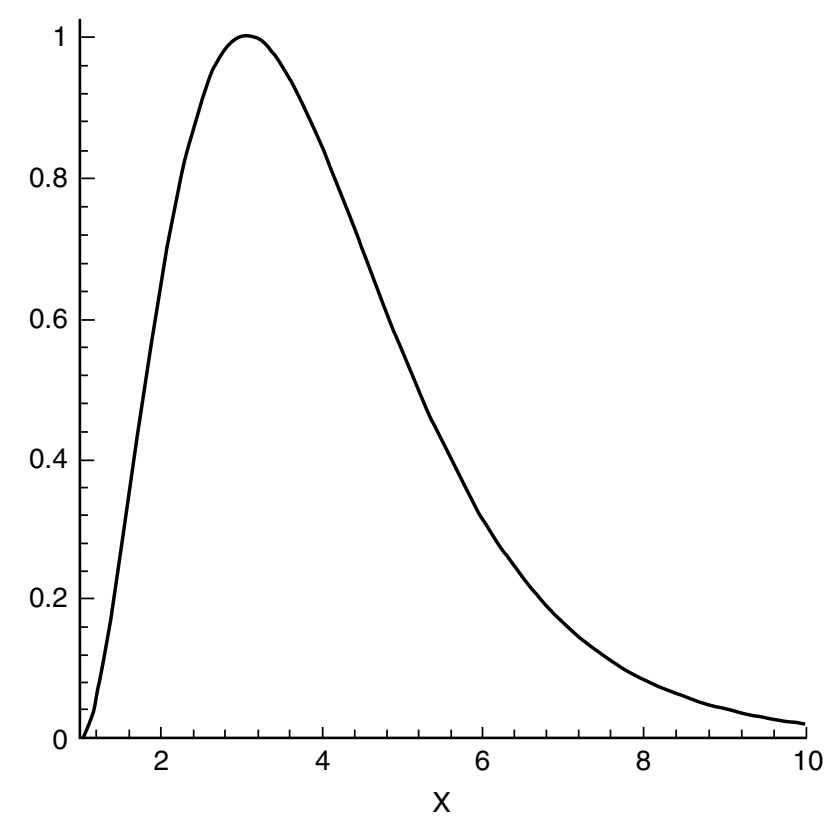

Figure 18. Time dependence of the mean population of the excited level of the detecting atom.

parameters of polarization. Following [97], consider first a fully polarized coherent plane wave in the weak quantum limit. To measure the Stokes parameters, one can use the six-port scheme shown in Fig. 19, which reflects the principal ideas of Mandel's operational approach to phase measurements [47]. A similar scheme has been analyzed [98]. The beam whose polarization is to be measured is first split by the non-polarizing beamsplitter $\mathrm{BS}_{1}$. One of the output beams is sent to a polarizing beamsplitter $\mathrm{PBS}_{1}$, which defines two linearly polarized orthogonal polarization eigenmodes labeled by $i=1,2$. Then, the intensities $I_{1}$ and $I_{2}$ are measured by the photodetectors. The other output beam from $\mathrm{BS}_{1}$ is further split at $\mathrm{BS}_{2}$ with the purpose of simultaneous measuring the sine and cosine of the phase difference between the polarized components of the field. One of the output beams from $\mathrm{BS}_{2}$ is analyzed with $\mathrm{PBS}_{2}$, which is oriented at $45^{\circ}$ with respect to the axes $i=1,2$ defined by $\mathrm{PBS}_{1}$. The other output beam from $\mathrm{BS}_{2}$ goes through a quarter-wave plate whose fast and slow axes are aligned along the $i=1,2$ directions. This beam is then analyzed at $\mathrm{PBS}_{3}$, whose axes are aligned with $\mathrm{PBS}_{2}$. 


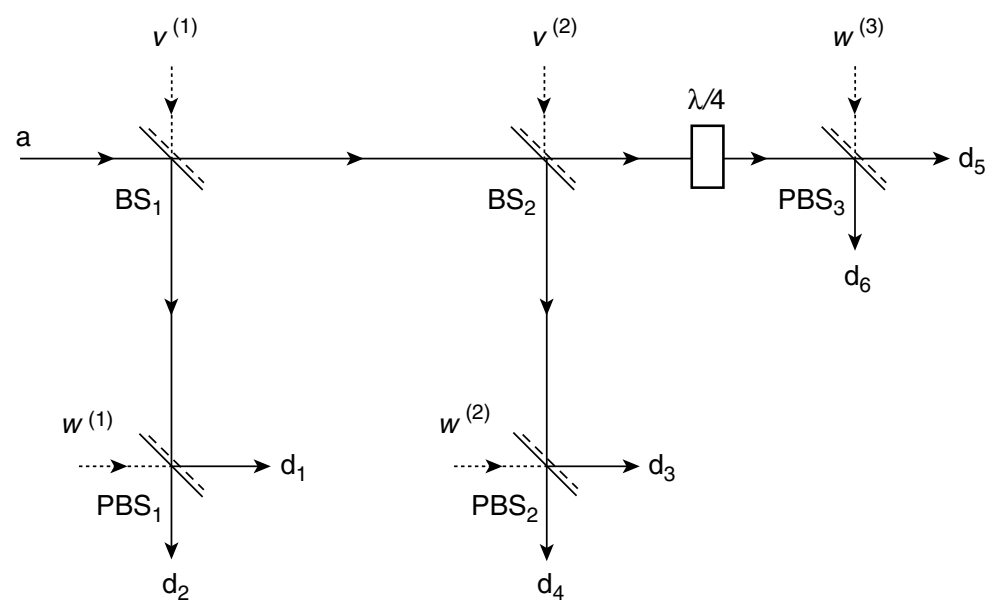

Figure 19. The experimental setup for measurement of the Stokes parameters.

Consider first the measurement of the classical field. Then, for $50 \%$ nonpolarizing beamsplitters, the detectors $d_{1}, \ldots d_{6}$ measures the intensities

$$
\begin{aligned}
& I_{1}=\frac{1}{2}\left|E_{1}\right|^{2} \\
& I_{2}=\frac{1}{2}\left|E_{2}\right|^{2} \\
& I_{3}=\frac{1}{4}\left(\left|E_{1}\right|^{2}+\left|E_{2}\right|^{2}\right)+2\left|E_{1}\right|^{2}\left|E_{2}\right|^{2} \cos \Delta \\
& I_{4}=\frac{1}{4}\left(\left|E_{1}\right|^{2}+\left|E_{2}\right|^{2}\right)-2\left|E_{1}\right|^{2}\left|E_{2}\right|^{2} \cos \Delta \\
& I_{5}=\frac{1}{4}\left(\left|E_{1}\right|^{2}+\left|E_{2}\right|^{2}\right)+2\left|E_{1}\right|^{2}\left|E_{2}\right|^{2} \sin \Delta \\
& I_{5}=\frac{1}{4}\left(\left|E_{1}\right|^{2}+\left|E_{2}\right|^{2}\right)-2\left|E_{1}\right|^{2}\left|E_{2}\right|^{2} \sin \Delta
\end{aligned}
$$

Where $I_{\ell}$ denotes the intensity measured by the detector $d_{\ell}$. From these relations, the classical Stokes parameters (130) can be found as

$$
\begin{array}{ll}
s_{0}^{\text {(plane) }}=I_{1}+I_{2}, & s_{1}^{\text {(plane) }}=I_{1}-I_{2} \\
s_{2}^{\text {(plane) }}=I_{2}-I_{4}, & s_{4}^{\text {(plane) }}=I_{5}-I_{6}
\end{array}
$$


were $1 \leftrightarrow x$ and $2 \leftrightarrow y$. Having defined our measurement in the classical domain, we next look at the quantum case when the unused ports in the beamsplitters are considered as input for vacuum fields [14]. These vacuum fields are indicated in Fig. 19 by $v_{\ell}$ and $w_{\ell}$ for BS and PBS, respectively.

The photon annihilation operators associated with the field at the output arms of $\mathrm{PBS}_{1}$ are related to those at the input through

$$
d_{1}=r a_{1}+t v_{1}^{(1)}, \quad d_{2}=r a_{2}+t c_{2}^{(1)}
$$

where $r=i / \sqrt{2}$ and $t=1 / \sqrt{2}$ are the field reflection and transmission coefficients, respectively, and $v_{\ell}^{(1)}$ describes the polarized vacuum field in the $\ell$ direction entering through the vacuum port of $\mathrm{BS}_{1}$ (cf. Section 4.6). The vacuum fields $w^{(k)}(k=1,2,3)$ do not couple with the measured field operators since they are orthogonal to them.

In analogy to the classical definition of the angular functions

$$
\sin \theta=2 \frac{\sqrt{I_{1} I_{2}}}{I_{1}+I_{2}}, \quad \cos \theta=\frac{I_{1}-I_{2}}{I_{1}+I_{2}}
$$

where $\theta$ is related to the ellipticity angle [87], we define the operators [97]

$$
S_{\theta}=2 \frac{\sqrt{n_{1} n_{2}}}{n_{1}+n_{2}}, \quad C_{\theta}=\frac{n_{1}-n_{2}}{n_{1}+n_{2}}
$$

Here

$$
n_{\ell} \equiv d_{\ell}^{+} d_{\ell}, \quad \ell=1,2
$$

are the photon number operators for the fields measured at the detectors 1 and 2 . From the Weyl-Heisenberg commutation relations for the photon operators, we get

$$
\left[S_{\theta}, C_{\theta}\right]=0
$$

so that these two quantities can be measured at once. It is also seen that

$$
S_{\theta}^{2}+C_{\theta}^{2}=\mathbf{1}
$$

On the other hand, at the output ports of $\mathrm{PBS}_{2}$ and $\mathrm{PBS}_{3}$, the field operators are

$$
\begin{aligned}
& d_{3}=\frac{1}{\sqrt{2}}\left[\left(\operatorname{tr}\left(a_{1}+a_{2}\right)+r^{2}\left(v_{1}^{(1)}+v_{2}^{(1)}\right)+t\left(v_{1}^{(2)}+v_{2}^{(2)}\right)\right]\right. \\
& d_{4}=\frac{-1}{\sqrt{2}}\left[\operatorname{tr}\left(a_{1}-a_{2}\right)+r^{2}\left(v_{1}^{(1)}-v_{2}^{(1)}\right)+t\left(v_{1}^{(2)}-v_{2}^{(2)}\right)\right] \\
& d_{5}=\frac{1}{\sqrt{2}}\left[r^{2}\left(i a_{1}+a_{2}\right)+\operatorname{tr}\left(i v_{1}^{(1)}+v_{2}^{(1)}\right)+r\left(i v_{1}^{(2)}+v_{2}^{(2)}\right]\right. \\
& d_{6}=\frac{-1}{\sqrt{2}}\left[r^{2}\left(i a_{1}-a_{2}\right)+\operatorname{tr}\left(i v_{1}^{(1)}-v_{2}^{(1)}\right)+r\left(i v_{1}^{(2)}-v_{2}^{(2)}\right]\right.
\end{aligned}
$$


Then, in analogy to the classical functions

$$
\begin{aligned}
\cos \Delta & =\frac{I_{3}-I_{4}}{\sqrt{\left(I_{3}-I_{4}\right)^{2}+\left(I_{5}-I_{6}\right)^{2}}} \\
\sin \Delta & =\frac{I_{5}-I_{6}}{\sqrt{\left(I_{3}-I_{4}\right)^{2}+\left(I_{5}-I_{6}\right)^{2}}}
\end{aligned}
$$

we get the operators

$$
\begin{aligned}
& C_{\Delta}=\frac{n_{3}-n_{4}}{\sqrt{\left(n_{3}-n_{4}\right)^{2}+\left(n_{5}-n_{6}\right)^{2}}} \\
& S_{\Delta}=\frac{n_{5}-n_{6}}{\sqrt{\left(n_{3}-n_{4}\right)^{2}+\left(n_{5}-n_{6}\right)^{2}}}
\end{aligned}
$$

such that

$$
\left[C_{\Delta}, S_{\Delta}\right]=0
$$

Here $n_{\ell}$ denotes the number operators constructed from the photon operators (183).

It is now a straightforward matter to calculate the variances of the operators (181) and (184) [97]. In particular, it is possible to show that the quantum fluctuations imply the uncertainty relations

$$
\begin{aligned}
& \left\langle\left(\Delta S_{2}^{\text {(plane) }}\right)^{2}\right\rangle\left\langle\left(\Delta C_{\Delta}\right)^{2}\right\rangle \geq \frac{1}{4}\left\langle S_{\Delta}\right\rangle \\
& \left\langle\left(\Delta S_{2}^{\text {(plane) }}\right)^{2}\right\rangle\left\langle\left(\Delta S_{\Delta}\right)^{2}\right\rangle \geq \frac{1}{4}\left\langle C_{\Delta}\right\rangle
\end{aligned}
$$

similar, in some sense, to the Susskind-Glogower uncertainty relations [41] and those of the operational definition of quantum phase [47]. Here $S_{2}^{(\text {plane })}$ is the Stokes operator obtained by the quantization of the Stokes parameter $s_{2}^{\text {(plane) }}$ in (179).

\section{Nondemolition Polarization Measurement}

In the preceding subsection, we discussed the polarization measurement through the use of the detection of the field variables done by photocounting techniques, which are field-destructive. As a result, successive measurements of the field variables yield different results. It seems to be tempting to use the measurement schemes avoiding backward action of the detecting device on the detected 
observable. Such a measurement is usually called a quantum nondemolition measurement [99]. Here, following our results [100], we discuss the possibility of quantum nondemolition measurement of polarization of the electromagnetic field via the Aharonov-Bohm effect [101].

First, we note that the standard photodetection is a local measurement of the field variables (intensities). At the same time, the Aharonov-Bohm effect represents a topological measurement referred to the properties of vector potential along some loop. In the usual form, the Aharonov-Bohm effect deals with static or slowly time-varying magnetic fields [101]. The effect consists in the appearance of a persistent current in a metallic loop over which the magnetic flux passes. This current is a periodic function of magnetic flux with the period of flux quantum $h c / e$. Besides that, certain resistance oscillations in the loop incorporated into an external circuit with the same period can occur.

An important case of varying magnetic field has been considered by Aronov et al. [102] under the assumption that the space-dependent time-varying electromagnetic field produces static electron energy minibands in the loop. These minibands have been suggested to appear due to electron motion in a time-averaged electrostatic potential periodic with coordinate along the loop circumference, produced by the square of time-varying electric field [103]. However, in the quantum case, an electron reflection forms an oscillating potential causes time-dependent phase shifts, resulting in an effective chaotization of the phase of electron wavefunction, except at energy multiples of $\hbar \omega$, where $\omega$ is the field frequency.

In our papers [100], we considered the case of optical frequencies $\omega>\Delta E / \hbar$, where $\Delta E$ is the width of the electron conduction band of the metal. Under this condition, the elastic scattering of electrons is prohibited if the separation between the conduction and higher nonoccupied bands of a metal is larger than $\hbar \omega$. In this case, the magnetic component of electromagnetic field represents the main source of the electron wavefunction phase shift. The effect of oscillating magnetic field results in the modulation of the electron transmission amplitude between the parts of the loop. As a result of the quantum interference of electron waves in oscillating potential, the dependence of the loop resistance of the time-varying field amplitude manifests a non-monotone character.

The geometry of the experiment assumes that the magnetic field oscillates along the axis orthogonal to the plane of the loop circumference and passing through its center. An example is provided by a small metallic ring surrounding an optical fiber. In this case, the largest contribution to the conductance oscillations comes from the $T E_{01}$ mode of the fiber field [100] (about the fiber modes; see Ref. 104). Definitely, such a measurement does not perturb the quantum state of the fiber mode. Another example is provided by the magnetic dipole radiation, when a longitudinal oscillating magnetic field can be observed, 
at least in some vicinity of the source. In the case of radioband frequencies, this vicinity seems to be extended enough to make a macroscopic measurement. As a powerful localized source of such a radiation the radioband Dicke superradiance [105] can be used. The geometry of the experiment is simplified, in this case, by a characteristic sharp radiation pattern of superradiance [64].

To make necessary estimations, consider a one-dimensional loop in a tightbinding approximation with two transmittance amplitudes $t_{1}$ and $t_{2}$ at the points $A$ and $B$, connecting two parts of the ring (see Fig. 20). It is supposed that $t_{1}, t_{2} \ll t_{0}$, the hopping amplitude between the nearest points inside the upper and lower parts of the ring. The system under consideration is described by the Hamiltonian [100]

$$
\begin{aligned}
H & =-t_{0} \sum_{n}\left(a_{n}^{+} a_{n+1}+b_{n}^{+} b_{n+1}\right)+\text { H.c. }+H_{\mathrm{int}} \\
H_{\mathrm{int}} & =-t_{1} a_{n_{1}}^{+} b_{n_{1}} e^{i \alpha_{1}}-t_{2} a_{n_{2}}^{+} b_{n_{2}} e^{i \alpha_{2}}+\text { H.c. }
\end{aligned}
$$

where $a, b$ are the electron (not photon) annihilation operators. The phases of transmission amplitudes at the contraction points $n_{i}$ are

$$
\alpha_{i}=\alpha_{i}^{0}+A_{i} \sin \left(\omega t+\delta_{i}\right)
$$

where $\alpha_{i}^{0}$ accounts for the effect of a static magnetic field applied perpendicular to the plane of the ring

$$
\alpha_{1}^{0}-\alpha_{2}^{0}=\frac{2 \pi \Phi_{S}}{\Phi_{0}}, \quad \Phi_{s}=\int \vec{B} \cdot d \vec{S}, \quad \Phi_{0}=\frac{h c}{e}
$$

while $A_{i}$ are the amplitudes of high-frequency field at corresponding points.

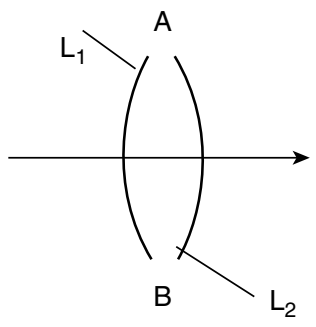

(a)

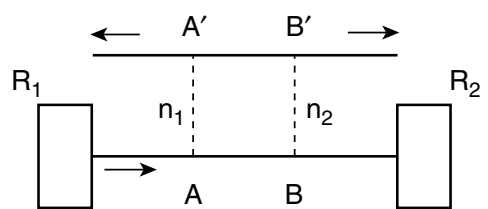

(b)

Figure 20. The scheme of one-dimensional Aharonov-Bohm loop, surrounding the direction of propagation of a longitudinal mode and weakly coupled at points $A$ and $B$ with the external leads $L_{1}$ and $L_{2}$ [(a) and (b)]. (d) The model of an ac normal-metal interferometer. $R_{1}$ and $R_{2}$ are the thermal reservoirs held at voltages $\pm V / 2$, respectively. 
The model with the Hamiltonian (186) can be solved exactly [100]. It is a straightforward matter to arrive at the following relation for the current

$$
J=\int_{0}^{\pi} \frac{d k}{2 \pi} W_{0}\left[\frac{W_{k}}{W_{0}+W_{k}}+\frac{W_{-k}}{W_{0}+W_{-k}}\right] \times\left[f_{0}\left(\epsilon_{k}-\frac{e V}{2}\right)-f_{0}\left(\epsilon_{k}+\frac{e V}{2}\right)\right]
$$

Here $f_{0}(\cdot)$ denotes the equilibrium distribution function of electrons, $\epsilon_{k}=$ $-2 t_{0} \cos k, W_{ \pm k}$ is the forward $(+k)$ or backward $(-k)$ scattering probability, and $V$ is the voltage. Then, the conductance $G$ can be found from (187) as follows:

$$
G=\frac{d J}{d V}
$$

It follows from (187) that the dependence of $G$ on the phase $\alpha$ and on the electromagnetic field amplitude leads to two different effects. First, the oscillatory dependence $G\left(\Phi_{S}\right)$ is the standard mesoscopic interference effect similar to that in static electron interferometer [101]. Another type of oscillating dependence $G(A)$ is completely caused by the time-varying field. The dependence of the conductance on the field intensity $P$ is shown in Fig. 21.

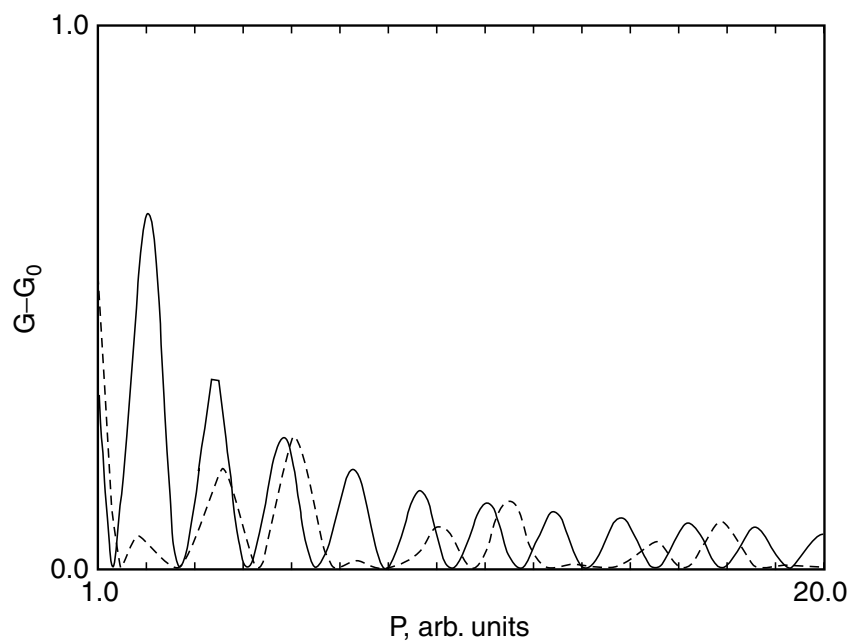

Figure 21. Direct-current conductance of the loop versus square root of ac power: solid line, $A_{1}: A_{2}=1: 1$; dotted line, $A_{1}: A_{2}=1: 2$. Change in the conductance is normalized with respect to static conductance oscillation amplitude. 
The observation of such oscillations requires temperatures low enough to allow the phase-breaking length of electron scattering $\ell_{\phi}$ to exceeds the circumference of the ring $L$. For the loop size of the order of $L=1 \mu \mathrm{m}$, which is technically reasonable, the condition $\ell_{\phi}>L$ is valid at $T \leq 1 \mathrm{~K}$.

At $L=1 \mu \mathrm{m}$, the measurement can be done at the magnetic field $B \sim 10^{-7} \mathrm{~T}$, which corresponds to the oscillating power $P \sim 10^{-3} \mathrm{~W}$ [100]. This seems to be not very high. Estimated in a different way as a minimum number of optical photons transmitted through the loop, the field should contain $n \sim 1 / \alpha \sim 137$ photons, where $\alpha \equiv e^{2} / \hbar c$ is the fine-structure constant. This estimation corresponds either to an optical soliton propagating through the fiber [104] or to the superradiant pulse in the radioband superradiance [105].

Thus, the linearly polarized longitudinal component of electromagnetic radiation arising in corresponding geometry (fiber or localized source) can be measured in nondemolition way with the aid of the Aharonov-Bohm effect at optical frequencies.

\section{E. Summary}

1. Localization of multipole photons at generation and absorption by an atom, described in terms of outgoing or incoming waves of photons, is compatible with Mandel's conception of localization.

2. The photon localization at detection leads to a strong increase of the vacuum noise in a certain vicinity of a detector (atom or active area of photodetector).

3. The two-atom scheme of the Hertz experiment with multipole photons, in which the radiation field is described by a superposition of outgoing and incoming waves focused on the emitting and detecting atoms respectively, obeys the causality principle even though the path of detecting photons is indefinite.

4. The quantum fluctuations of polarization can be measured in the multiport six-detector scheme similar to that proposed by Mandel for the phase measurements.

5. The linear polarization of longitudinal component of electromagnetic field caused by a characteristic geometry of the system (cylindrical geometry in an optical fiber or spherical in the case of field generated by a local source) can be measured in a nondemolition way with the aid of the Aharonov-Bohm effect.

\section{CONCLUSION}

Every problem becomes very childish when once it is explained to you. —Sir Arthur Conan Doyle, The Dancing Men 
In this chapter we have reviewed some results concerning the quantum multipole radiation. Although the representation of quantum electromagnetic radiation in terms of spherical waves of photons known since the first edition in 1936 of the famous book by Heitler on quantum theory of radiation [2], where this subject is discussed in the Appendix, this representation is not a widespread one. The spherical waves of photons are considered in very few advanced monographs on quantum optics [26]. The brilliant encyclopedic monographs [14,15] just touch on the subject.

At the same time, the quantum multipole radiation is precisely what the atomic transitions between the states with given angular momenta emit. The states of multipole (spherical) photons are specified by given angular momentum and projection of the angular momentum, while the spin state (polarization) is changed in spacetime. On the contrary, the common representation of plane waves of photons is specified by a given linear momentum and polarization everywhere. This difference reflects the boundary conditions used in the canonical quantization of the free electromagnetic field [2].

It was shown in Section I that the clear-cut distinction between the symmetry properties of plane and spherical waves leads to a qualitative difference in the zero-point oscillations. While the former are homogeneous in space (along the direction of propagation), the latter concentrate in a certain vicinity of a local source (atom) where they may exceed the level calculated in the model of plane waves. Although the result is simple in itself, to our knowledge, it was indicated for the first time in 1999 [46] and then discussed later [22,29]. Since the zeropoint oscillations define the quantum limit of precision of measurements of corresponding physical quantity [14,15,99], this result seems to be very important. The estimations based on the results of Section II.B show that the zero-point oscillations exceed the conventional level provided by the representation of plane waves at the distances $r \leq 0.2 \lambda$, where $\lambda$ is the wavelength. Within the standard classification, it corresponds to the so-called intermediate zone. Let us stress that this distance is of the order of the typical interatomic separation in experiments with trapped Ridberg atoms [32].

One of the major trends of current research is the study of transmission of "information" between the atom and photons in the process of emission and absorption. In particular, the conservation of angular momentum provides the transmission of the "quantum phase information" in the atom-field system. The atomic quantum phase can be constructed as the $S U(2)$ phase of the angular momentum of the excited atomic state (Section III). It is shown that this phase has very close connection with the EPR paradox and entangled states in general. Via the integrals of motion, it is mapped into the Hilbert space of multipole photons (Section IV.A). This mapping is adequately described by the dual representation of multipole photons, constructed in another study [46] (see also Section IV.B, below). Instead of the quantum number $m$, corresponding to the 
projection of angular momentum, these photons are specified by the quantum phase index. The spectrum of corresponding quantum (radiation) phase is discrete and lies in the interval $(0,2 \pi)$. In the classical limit provided by the high-intensity coherent multipole radiation, the eigenvalues of the radiation phase are distributed uniformly over this interval. By construction, the radiation phase is complementary to Mandel's operational phase [47]. It defines the quantum phase in terms of what can be generated by a quantum local source. In the quantum limit of weak intensity, the behavior of radiation and the PeggBarnett [45] quantum phases are completely different. They are caused by the specific truncation procedure used within the Pegg-Barnett approach, which leads to an effective violation of the Weyl-Heisenberg algebra of photons.

The radiation phase is closely related to the polarization of the multipole field. In contrast to the plane waves, the polarization of multipole radiation is specified by three orthogonal directions of the field oscillations or by three allowed spin states of photons [28]. Therefore, the polarization is described by the $(3 \times 3)$ Hermitian matrix of polarization (operator matrix, in the quantum case) (Sections V.A and V.B). This general form of the polarization matrix gives in the limit the standard case of $(2 \times 2)$ matrix in the representation of plane waves. The set of multipole Stokes operators corresponds to a representation of the $S U(3)$ subalgebra in the Weyl-Heisenberg algebra of photons. The Cartan algebra of this $S U(3)$ subalgebra corresponds to the representation of the radiation phase in the case of the angular momentum $j=1$. Thus, the polarization of multipole radiation has a certain inherent quantum phase that, in some important cases, can be interpreted as the phase difference between the components with different polarization (Section V.B). The quantum properties of polarization can be measured with the aid of a multiport scheme [97] (Section VI.C).

The polarization and quantum phase properties of multipole photons change with the distance from the source. This dependence can be adequately described with the aid of the local representation of the photon operators proposed in Ref. 91 and discussed in Section V.D. In this representation, the photon operators of creation and annihilation correspond to the states with given spin (polarization) at any point. This representation may be useful in the quantum near-field optics. As we know, so far near-field optics is based mainly on the classical picture of the field [106].

The local representation of multipole photons is compatible with the Mandel operational definition of photon localization [20]. In addition to the localization at photodetection, it permits us to describe a complete Hertz-type experiment with two identical atoms used as the emitter and detector (Section VI.A). Although the photon path is undefined from the quantum-mechanical point of view, the measurement process in such a system obeys the causality principle (Section IV.B). The two-atom Hertz experiment can be realized for the trapped 
Ridberg atoms. Let us stress that such a measurement is closely connected with the problem of atomic entanglement discussed in Ref. 76.

The standard measurement of different properties of quantum electromagnetic radiation is based on the photodetection, which is field destructive. Following our consideration of the possibility of the Aharonov-Bohm effect at optical frequencies [100], we propose here a new nondemolition method of polarization measurement in which the linearly polarized longitudinal mode of the field is detected without any perturbation of its quantum state (Section VI.D). The estimation of physical conditions shows that such a measurement can be done either for the photons propagating through the fiber, or for the superradiant photons in radioband frequencies.

In this review, we relied to a large extent on our own results. We believe, however, that this chapter covers a number of topics important for quantum optics, and that the reported results may stimulate an interest in further investigations. Success in the field of quantum optics has convicingly shown that developments in optics have very often had a direct influence on other fields, both pure and applied.

\section{Acknowledgments}

A number of reported results were obtained together with my colleagues O. Aytur, T. Hakioğlu, A. A. Klyachko, and B. Tanatar and students Ö. E. Müstecaplığlu, M. Ünsal, and M. A. Can, whom I would like to thank for fruitful collaboration. I wish to express a deep gratitude to A. Bandilla, H.D. Doebner, J. H. Eberly, E. Kapuscik, P. L. Knight, P. Kumar, A. Miranowicz, H. Paul, V. I. Rupasov, A. Vourdas, H. Walther, K. Wodkiewicz, and A. Wuensche for stimulating discussions. Finally, I am grateful to my wife, Natalia Shumovsky, and Dr. A. I. Degtyarev, who helped me a lot in preparing a readable text.

\section{References}

1. P. A. M. Dirac, Proc. Roy. Soc. Lond. A 114, 243, 710 (1927).

2. W. Heitler, The Quantum Theory of Radiation, Oxford Univ. Press, New York, 1954.

3. N. N. Bogoliubov and D.V. Shirkov, Introduction to the Theory of Quantum Fields, Wiley, New York, 1980.

4. V. B. Berestetskii, E. M. Lifshitz, and L. P. Pitaevskii, Quantum Electrodynamics, Pergamon, Oxford, 1982.

5. V. Weisskopf and E. P. Wigner, Z. Phys. 63, 54 (1930); 65, 18 (1930).

6. W. E. Lamb Jr. and R. C. Retherford, Phys. Rev. 72, 241 (1947).

7. H. B. G. Casimir, J. de Chem. Physique 46, 407 (1949); H. B. G. Casimir and D. Polder, Phys. Rev. 73, 360 (1948).

8. W. W. Chow, M. O. Scully, and J. O. Stoner Jr., Phys. Rev. A 11, 1380 (1975); R. M. Herman, H. Groth, R. Kornblith, and J. H. Eberly, Phys. Rev. A 11, 1389 (1975).

9. D. Stoler, Phys. Rev. D 1, 3217 (1970); H. P. Yuen, Phys. Rev. A 13, 2226 (1976); D. F. Walls, Nature 106, 141 (1983); R. Loudon and P. Knight, J. Mod. Optics 34, 709 (1987).

10. H. A. Kramers, Quantum Mechanics, North-Holland, Amsterdam, 1985.

11. E. A. Power, Introductory Quantum Electrodynamics, Longman, London, 1964. 
12. I. Bialynicki-Birula and Z. Bialanicka-Birula, Quantum Electrodynamics, Pergamon, Oxford, 1976.

13. M. W. Evans and J.-P. Vigier, The Engimatic Photon, Vols. 1, 2, Kluwer, Dordrecht, 1994; M. W. Evans, J.-P. Vigier, S. Roy, S. Jeffers, and G. Hunter, The Engimatic Photon, Vols. 3, 4, Kluwer, Dordrecht, 1997.

14. L. Mandel and E. Wolf, Optical Coherence and Quantum Optics, Cambridge Univ. Press, New York, 1995.

15. M. O. Scully and M. S. Zubairy, Quantum Optics, Cambridge Univ. Press, New York, 1997.

16. T. D. Newton and E. P. Wigner, Rev. Mod. Phys. 21, 400 (1949).

17. R. Acharya and E. C. G. Sudarshan, J. Math. Phys. 1, 532 (1960).

18. I. Bialynicki-Birula, Acta Phys. Pol. A 86, 97 (1994).

19. I. Bialynicki-Birula, Phys. Rev. Lett. 80, 5247 (1998).

20. L. Mandel, Phys. Rev. 144, 1071 (1966).

21. S. John, Phys. Rev. Lett. 58, 2486 (1987).

22. A. S. Shumovsky, in Electronic Proc. 7th Central Eur. Workshop on Quantum Optics April 28May 1, 2000, available at http://optics.szfki.kfki.hu/cewqo2000.

23. E. U. Condon and G. H. Shortley, The Theory of Atomic Spectra, Cambridge Univ. Press, New York, 1987.

24. H. Bateman, The Mathematical Analysis of Electric and Optical Wave-Motion Dover, New York, 1955.

25. J. D. Jackson, Classical Electrodynamics, Wiley, New York, 1978.

26. C. Cohen-Tannouji, J. Dupont-Roc, and G. Grinberg, Atom-Photon Interaction, Wiley, New York, 1992.

27. A. S. Davydov, Quantum Mechanics, Pergamon, Oxford, 1976.

28. A. S. Shumovsky and Ö. E. Müstecaplığlu, Phys. Rev. Lett. 80, 1202 (1998).

29. A. S. Shumovsky, lanl e-print quantum-ph/0007109.

30. P. W. Milony, The Quantum Vacuum, Academic, San Diego, 1994.

31. G. Compagno, R. Pasante, and F. Persico, Atom-field Interaction and Dressed Atoms, Cambridge Univ. Press, Cambridge, UK, 1995.

32. S. Haroche, AIP Conf. Proc. Vol. 464, Issue 1, p. 45 (1999); J. M. Raimond, E. Hagley, X. Maitre, G. Nogues, C. Wunderlich, M. Brune, and S. Haroshe, in AIP Conf. Proc. Vol. 477, Issue 1, p. 209 (1999).

33. M. Weidinger, B. T. H. Varcoe, R. Heerlein, and H. Walther, Phys. Rev. Lett. 82, 3795 (1999).

34. A. K. Ekert, Phys. Rev. Lett. 67, 661 (1991); C. H. Benett and S. J. Wiesner, Phys. Rev. Lett. 69, 2881 (1992); C. H. Benett, G. Brassard, C. Crepeau, R. Jozza, A. Peres, and W. K. Wootters, Phys. Rev. Lett. 70, 1895 (1993); D. Bouwmeester, J.-W. Pan, K. Mattle, M. Eibl, H. Weinfürter, and A. Zeilinger, Nature 390, 575 (1997); D. Boschi, S. Branca, F. De Martini, L. Hardy, and S. Popescu, Phys. Rev. Lett. 80, 1121 (1998); J.-W. Pan, D. Bowmeester, H. Weinfürter, and A. Zeilinger, Phys. Rev. Lett. 80, 3891 (1998).

35. C. Adlard, E. P. Pike, and S. Sarkar, Phys. Rev. Lett. 79, 1583 (1997).

36. A. S. Shumovsky, Opt. Commun. 136, 219 (1997).

37. Physica Scripta T 48, special issue (1995).

38. R. Lynch, Phys. Rep. 256, 367 (1995).

39. R. Tanaś, A. Miranowicz, and T. Gantsog, Prog. Opt. 35, 1 (1996). 
40. D. T. Pegg and S. M. Barnett, J. Mod. Opt. 44, 225 (1997).

41. L. Susskind and J. Glogower, Physics 1, 49 (1964).

42. S. M. Barnett and D. T. Pegg, J. Phys. A 19, 3849 (1986).

43. A. Lukš and V. Perinova, Czech. J. Phys. A 41, 1205 (1991).

44. W. H. Louisell, Phys. Lett. 7, 60 (1963); P. Carruthers and M. Nieto, Rev. Mod. Phys. 40, 411 (1968).

45. D. T. Pegg and S. M. Barnett, Europhys. Lett. 6, 483 (1988); S. M. Barnett and D. T. Pegg, J. Mod. Opt. 36, 7 (1989); D. T. Pegg and S. M. Barnett, J. Mod. Opt. 39, 2121 (1992).

46. A. S. Shumovsky, J. Phys. A 32, 6589 (1999).

47. J. W. Noh, A. Fougères, and L. Mandel, Phys. Rev. Lett. 67, 1426 (1991); J. W. Noh, A. Fougères, and L. Mandel, Phys. Rev. A 45, 242 (1992).

48. A. Fougères, J. R. Torgerson, and L. Mandel, Optics Commun. 105, 199 (1994); J. R. Torgerson and L. Mandel, Phys. Rev. Lett. 76, 3939 (1996); J. R. Torgerson and L. Mandel, Optics Commun. 133, 153 (1997).

49. A. Bandilla and H. Paul, Ann. Phys. (Leipzig) 23, 323 (1969); W. P. Schleih, A. Bandilla, and H. Paul, Phys. Rev. A 45, 6652 (1992); M. Freyberger, K. Vogel, and W. P. Schleih, Phys. Lett. A 176, 41 (1993); U. Leonhardt and H. Paul, Phys. Rev. A 47, R2460 (1993).

50. B.-G. Englert and J. Wodkiewicz, Phys. Rev. A 51, R266 (1995).

51. A. Vourdas, Phys. Rev. A 41, 1653 (1990).

52. J.-P. Serre, Linear Representations of Finite Groups, Springer, New York, 1977.

53. A. S. Shumovsky and Ö. E. Müstecaplığlu, Phys. Lett. A 235, 438 (1997); A. S. Shumovsky and Ö. E. Müstecaplıoğlu, in G. Hunter, S. Jeffers, and J.-P. Vigier (Eds.), Causality and Locality in Modern Physics, Kluwer, Dordrecht, 1998.

54. A. S. Shumovsky and Ö. E. Müstecaplıoğlu, J. Mod. Opt. 45, 619 (1998); A. S. Shumovsky and Ö. E. Müstecaplığlu, Opt. Commun. 146, 124 (1998).

55. J. M. Levi-Leblond, Ann. Phys. (NY) 101, 319 (1979); S. M. Barnett and D. T. Pegg, Phys. Rev. A 41, 3427 (1990); L. L. Sanchez-Soto and A. Luis, Optics Commun. 105, 84 (1994); A. Lukš and V. Perinova, Quantum Opt. 6, 125 (1994).

56. I. C. Koo and J. H. Eberly, Phys. Rev. A 14, 2174 (1976).

57. M. Born and E. Wolf, Principles of Optics, Pergamon, Oxford, 1970.

58. W. H. Louisell, Radiation and Noise in Quantum Electronics, McGraw-Hill, New York, 1964.

59. R. Loudon, The Quantum Theory of Light, Oxford Univ. Press, New York, 1973.

60. L. D. Landau and E. M. Lifshitz, The Classical Theory of Fields, Pergamon, Oxford, 1975.

61. G. Herzberg, Atomic Spectra and Atomic Structure, Dover, New York, 1944; C. E. Moore, Atomic Energy Levels (U.S. National Bureau of Standards, Washington DC, 1971); J. M. Hollas, Modern Spectroscopy, Wiley, New York, 1996.

62. B. Crosignani, P. Di Porto, and M. Bertolotti, Statistical Properties of Scattered Light, Academic, New York, 1975; B. E. A. Saleh, Photoelectron Statistics with Applications to Spectroscopy and Communications, Springer, Berlin, 1978; J. Perrrina, Quantum Statistics of Linear and Nonlinear Optical Phenomena, Reidel, Dordrecht, 1984; N. N. Bogolubov, A. S. Shumovsky, and V. I. Yukalov (Eds.), Interaction of Electromagnetic Field with Condensed Matter, World Scientific, Singapore, 1990; A. S. Shumovsky, in T. Hakiioğlu and A. S. Shumovsky (Eds.), Quantum Optics and Spectroscopy of Solids, Kluwer, Dordrecht, 1997. 
63. A. S. Shumovsky and B. Tanatar, Phys. Rev. A 48, 4735 (1993); Ö. E. Müstecaplığlu and A. S. Shumovsky, Appl. Phys. Lett. 70, 3489 (1997); Ö. E. Müstecaplığlu and A. S. Shumovsky, Phys. Rev. B 60, 3970 (1999).

64. A. Allen and J. H. Eberly, Optical Resonance and Two-Level Atoms, Wiley, New York, 1975.

65. A. Einstein, Z. Phys. 18, 121 (1917).

66. D. Meschede, H. Walther, and G. Muller, Phys. Rev. Lett. 54, 551 (1985); S. Haroche and J. M. Raimond, in D. Bates and B. Bederson (Eds.), Advances in Atomic and Molecular Physics, Vol. 20, Academic, New York, 1985; G. Rempe, H. Walther, and N. Klein, Phys. Rev. Lett. 58, 353 (1987); E. R. Pike and H. Walther (Eds.), Photons and Quantum Fluctuations, Hilger, Bristol, 1988; M. O. Scully, B.-G. Englert, and H. Walther, Nature 351, 111 (1991); G. Raithel, O. Benson, and H. Walther, Phys. Rev. Lett. 72, 3506 (1995); H. Walther, in T. Hakioğlu and A. S. Shumovsky (Eds.), Quantum Optics and the Spectroscopy of Solids, Kluwer, Dordrecht, 1997.

67. E. T. Jaynes and F. W. Cummings, Proc. IEEE 51, 89 (1963).

68. H. I. Yoo and J. H. Eberly, Phys. Rep. 118, 239 (1985); F. L. Kien and A. S. Shumovsky, Int. J. Mod. Phys. B 5, 2287 (1991); R. Shore and P. L. Knight, J. Mod. Optics 40, 1195 (1993); E. I. Aliskenderov, A. S. Shumovsky, and H. T. Dung, Phys. Part. Nucl. 24, 177 (1993).

69. V. I. Rupasov, JETP Lett. 36, 142 (1982); V. I. Rupasov, Sov. Phys. JETP 56, 989 (1982); V. I. Rupasov and V. I. Yudson, Sov. Phys. JETP 60, 927 (1984); V. I. Rupasov and M. Singh, Phys. Rev. Lett. 77, 338 (1996); V. I. Rupasov and M. Singh, Phys. Rev. A 54, 3614 (1996).

70. I. S. Gradshtein and I. M. Ryzhik, Table of Integrals, Series, and Products, Academic, Boston, 1994.

71. D. M. Brink and G. R. Satchler, Angular Momentum, Clarendon, Oxford, 1993.

72. H. S. M. Coxeter and W. O. J. Moser, Generators and Relations for Discrete Groups, Springer, Berlin, 1965.

73. A. Einstein, B. Podolsky, and N. Rosen, Phys. Rev. 47, 777 (1935).

74. D. Bohm, Quantum Theory, Prentice-Hall, Englewood Cliffs, NJ, 1951.

75. J. S. Bell, Physics 1, 195 (1964); J. S. Bell, Rev. Mod. Phys. 38, 447 (1966).

76. M. B. Plenio, S. F. Huelga, A. Beige, and P. L. Knight, Phys. Rev. A 59, 2468 (1999).

77. H. Frölich, Phys. Rev. 79, 845 (1950); J. Bardeen, L. N. Cooper, and J. R. Schrieffer, Phys. Rev. 108, 1175 (1957); N. N. Bogolubov, Sov. Phys. JETP 7, 41 (1958); J. R. Schrieffer, Superconductivity, Benjamin, New York, 1964.

78. P. W. Anderson, Phys. Rev. 110, 985 (1958).

79. W. Thirring, Comm. Math. Phys. 7, 181 (1968); N. N. Bogolubov Jr., B. I. Sadovnikov, and A. S. Shumovsky, Mathematical Methods of Statistical Mechanics of Model Systems, CRC Press, Boca Raton, FL, 1994.

80. N. N. Bogolubov, A. M. Kurbatov, and A. S. Shumovsky (Eds.), N. N. Bogolubov Selected Works, Part II, Quantum and Classical Statistical Mechanics, Gordon \& Breach, New York, 1991.

81. R. J. Glauber, Phys. Rev. 131, 2766 (1963); E. C. G. Sudarshan, Phys. Rev. Lett. 10, 277 (1963).

82. A. Luis, A. L. L. Sanchez-Soto, and R. Tanaś, Phys. Rev. A 51, 1634 (1995).

83. Ts. Gantsog and R. Tanaś, J. Mod. Optics 38, 1537 (1991).

84. T. Hakioğlu, J. Phys. A 31, 707 (1998).

85. R. Hanbury-Brown and R. Q. Twiss, Phil. Mag. 45, 663 (1954); H. Hanbury-Brown and R. Q. Twiss, Nature 178, 1046 (1956); H. Hanbury-Brown and R. Q. Twiss, Proc. Roy. Soc. A 242, 300 (1957); R. Hanbury-Brown, The Intensity Interferometer, Taylor \& Fancis, London, 1974. 
86. W. Vogel and D.-G. Welsch, Lectures on Quantum Optics, Akademic, Verlag, Berlin, 1994.

87. R. M. A. Azzam and N. M. Bashara, Ellipsometry and Polarized Light, North-Holland, Amsterdam, 1987; D. S. Kliger, J. W. Lewis, and C. E. Randal, Polarized Light in Optics and Spectroscopy, Academic, New York, 1990; S. Huard, Polarization of Light, Wiley, New York, 1996; C. Brosseau, Fundamentals of Polarized Light, Wiley, New York, 1998.

88. A. S. Shumovsky, Laser Phys. 9, 26 (1999); A. S. Shumovsky, Ö. E. Müstecaplığlu, and M. Ünsal, Laser Phys. 10, 31 (2000).

89. E. Wolf, Nuovo Cimento 12, 884 (1954).

90. J. M. Jauch and F. Rohrlich, The Theory of Photons and Electrons (Addison-Wesley, Reading, MA, 1959).

91. A. S. Shumovsky and A. A. Klyachko, Laser Phys. 11, 57 (2001).

92. R. J. Cook, Phys. Rev. A 25, 2164 (1982); R. J. Cook, in L. Mandel and E. Wolf (Eds.), Coherence and Quantum Optics, Vol. V, (Plenum, New York, 1984; E. R. Pike and S. Sarkar, in E. R. Pike and S. Sarkar (Eds.), Frontiers in Quantum Optics, Hilger, Bristol, 1986.

93. R.P Feynman and A. R. Hibbs, Quantum Mechanics and Path Integrals McGrow-Hill, New York, 1965.

94. E. H. Haug and J. A. Støvneng, Rev. Mod. Phys. 61, 917 (1989); R. Landauer, Nature 341, 567 (1989); R. Landauer and Th. Martin, Rev. Mod. Phys. 66, 217 (1994).

95. A. Ranfagni, D. Mugnai, P. Fabeni, and G. Pazzi, Appl. Phys. Lett. 58, 774 (1991); A. Enders and G. Nimitz, J. Phys. (France) I 2, 1693 (1992); A. Ranfagni, P. Fabeni, G. Pazzi, and D. Mugnai, Phys. Rev. E 48, 1453 (1993); C. Spielmann, R. Szipöcs, A. Stingl, and F. Kraus, Phys. Rev. Lett. 73, 2308 (1994); D. Mugnai, A. Ranfagni, and R. Ruggeri, Phys. Rev. Lett. 84, 4830 (2000).

96. D. Kaup and V. I. Rupasov, J. Phys. A 29, 6911 (1996).

97. T. Hakioğlu, A. S. Shumovsky, and O. Aytur, Phys. Lett. A 194, 304 (1994).

98. A. Zuchetti, W. Vogel, and D.-G. Welsch, Phys. Rev. A 54, 856 (1996).

99. V. B. Braginsky, Y. I. Vorontsov, and F. I. Khalili, Sov. Phys. JETP 46, 705 (1977); C. M. Caves, K. S. Thorn, R. W. D. Drever, V. D. Sandberg, and M. Zimmerman, Rev. Mod. Phys. 52, 341 (1980); V. B. Braginsky and F. I. Khalili, Quantum Measurement, Cambridge Univ. Press, New York, 1992.

100. I. O Kulik and A. S. Shumovsky, Appl. Phys. Lett. 69, 2779 (1996); I. O Kulik and A. S. Shumovsky, in G. Hunter, S. Jeffers, and J.-P. Vigier (Eds.), Causality and Locality in Modern Physics, Kluwer, Dordrecht, 1998.

101. Y. Aharonov and D. Bohm, Phys. Rev. 115, 485 (1959); M. Peshkin and A. Tonomura, The Aharonov-Bohm Effect, Springer Verlag, Berlin, 1989; B. L. Altshuler, P. A. Lee, and R. A. Webb (Eds.), Mesoscopic Phenomena in Solids, North Holland, Amsterdam, 1991.

102. I. E. Aronov, E. N. Bogachek, and I. V. Krive, Phys. Lett. A 164, 331 (1992); I. E. Aronov, A. Grincwajg, M. Jonson, R. I. Shekhter, and E. N. Bogachek, Solid State Commun. 91, 75 (1994).

103. V. Chandrasekhar, R. A. Webb, M. J. Brady, M. B. Ketchen, W. J. Gallagher, and A. Kleinsasser, Phys. Rev. Lett. 70, 2020 (1991).

104. G. Agrawal, Nonlinear Fiber Optics, Academic, Boston, 1989.

105. Yu. Kiselev, A. Prutkoglyad, A. Shumovsky, and V. Yukalov, Sov. Phys. JETP 94, 344 (1988); Yu. F. Kiselev, A. S. Shumovsky, and V. I. Yukalov, Mod. Phys. Lett. B 3, 1149 (1989).

106. D. W. Pohl and D. Courjon (Eds.), Near Field Optics, Kluwer, Dordrecht, 1993. 